\title{
Antiparasitic Activity of Sulfur- and Fluorine-Containing Bisphosphonates against Trypanosomatids and Apicomplexan Parasites
}

\author{
Tamila Galaka ${ }^{1}$, Mariana Ferrer Casal ${ }^{1}$, Melissa Storey ${ }^{2}$, Catherine Li $^{2}$, María N. Chao ${ }^{1}$, \\ Sergio H. Szajnman ${ }^{1}$, Roberto Docampo ${ }^{2}$, Silvia N. J. Moreno ${ }^{2}$ and Juan B. Rodriguez ${ }^{1, *}$ \\ 1 Departamento de Química Orgánica and UMYMFOR (CONICET-FCEyN), Facultad de Ciencias Exactas y \\ Naturales, Universidad de Buenos Aires, Pabellón 2, Ciudad Universitaria, C1428EHA Buenos Aires, \\ Argentina; galakatamila@qo.fcen.uba.ar (T.G.); mferrer@qo.fcen.uba.ar (M.F.C.); \\ nchao@qo.fcen.uba.ar (M.N.C.); shs@qo.fcen.uba.ar (S.H.S.) \\ 2 Center for Tropical and Emerging Global Diseases and Department of Cellular Biology, \\ University of Georgia, Athens, GA 30602, USA; mtay104@uga.edu (M.S.); catli@uga.edu (C.L.); \\ rdocampo@uga.edu (R.D.); smoreno@uga.edu (S.N.J.M.) \\ * Correspondence: jbr@qo.fcen.uba.ar; Tel.: +54-11-4576-3385; Fax: +54-11-4576-3346
}

Academic Editor: Artur Silva

Received: 31 October 2016; Accepted: 30 December 2016; Published: 4 January 2017

\begin{abstract}
Based on crystallographic data of the complexes 2-alkyl(amino)ethyl-1,1-bisphosphonatesTrypanosoma cruzi farnesyl diphosphate synthase, some linear 1,1-bisphosphonic acids and other closely related derivatives were designed, synthesized and biologically evaluated against $T$. cruzi, the responsible agent of Chagas disease and against Toxoplasma gondii, the etiologic agent of toxoplasmosis and also towards the target enzymes farnesyl pyrophosphate synthase of T. cruzi (TcFPPS) and T gondii ( $T g$ FPPS), respectively. The isoprenoid-containing 1,1-bisphosphonates exhibited modest antiparasitic activity, whereas the linear $\alpha$-fluoro-2-alkyl(amino)ethyl-1,1-bisphosphonates were unexpectedly devoid of antiparasitic activity. In spite of not presenting efficient antiparasitic activity, these data turned out to be very important to establish a structural activity relationship.
\end{abstract}

Keywords: bisphosphonates; farnesyl diphosphate synthase; Trypanosoma cruzi; Toxoplasma gondii; Chagas disease; toxoplasmosis; antiparasitic agents

\section{Introduction}

American Trypanosomiasis (Chagas disease) is a chronic parasitosis caused by the kinetoplastid parasite Trypanosoma cruzi and is the largest parasitic disease burden of the Americas [1,2]. Isoprenoids are essential compounds of the cellular machinery of all organisms due to their roles in a variety of biological processes. Several enzymes of this pathway in T. cruzi, involved in the synthesis of sterols [1,2] and farnesyl diphosphate [3] have been reported to be excellent targets against pathogenic parasites. Despite their structural and functional variety, all isoprenoids derive from a common precursor: isopentenyl diphosphate (IPP), and its isomer, dimethylallyl diphosphate (DMAPP). In T. cruzi, IPP is synthesized only via the so-called mevalonate pathway, which has the 3-hydroxy-3-methylglutaryl-CoA (HMG-CoA) reductase as the key regulatory enzyme [4]. In Apicomplexan parasites such as Toxoplasma gondii, the responsible agent of toxoplasmosis, IPP is synthesized by the prokaryotic-type 1-deoxy-D-xylulose-5-phosphate (DOXP) pathway [5], which localizes to the apicoplast and is essential [6]. T. gondii possesses a bifunctional farnesyl diphosphate synthase (FPPS)/geranyl diphosphate synthase (GGPPS) (TgFPPS) that is able to catalyze the formation of both FPP and GGPP $[7,8]$. It is very relevant to state that it is possible to inhibit the host mevalonate pathway and the TcFPPS or DOXP pathway of T. gondii synergistically [9]. Certainly, 
the use of two approved and widely used drugs, zoledronic acid and atorvastatin exhibited a strong synergism in the inhibition of T. gondii growth [9]. In addition, TcFPPS has 50\% identity with $T g$ FPPS, therefore, it would be possible to access very selective inhibitors of the activity of each enzyme [10].

Bisphosphonates (2) are metabolically stable pyrophosphate analogs (1) in which a methylene group replaces the oxygen atom bridge between the two phosphorus atoms of the pyrophosphate moiety. Substitution at the carbon atom with different side chains has generated a large family of compounds. Several bisphosphonates are potent inhibitors of bone resorption and are in clinical use for the treatment and prevention of osteoporosis, Paget's disease, hypercalcemia, tumor bone metastases, and other bone diseases [11,12]. Selective action on bone is based on the binding of the bisphosphonate moiety to the bone mineral [13]. Apart from their ability as inhibitors of bone resorption, bisphosphonates have turned out to be antibacterial agents [13], anticancer agents [14,15], selective inhibitors of acid sphingomyelinase [16], and stimulators of $\gamma \delta \mathrm{T}$ cells [17]. Interestingly, these compounds behave as antiparasitic agents as well [10,18]. Aminobisphosphonates such as pamidronate (3), alendronate (4), and risedronate (5) were first found to be effective in the inhibition of T. cruzi in vitro and in vivo without toxicity to the host cells (Figure 1) [19]. In addition, the scope of these compounds was broadened by finding that some bisphosphonates were also growth inhibitors of T. gondii, T. brucei rhodesiense, Leishmania donovani and Plasmodium falciparum [20-22]. In vivo testing in mice has shown that risedronate can have a significantly marked protective effect in mice infected by $T$. cruzi $[23,24]$.

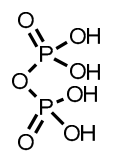

1

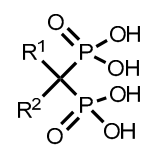

2

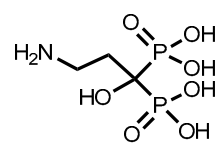

3

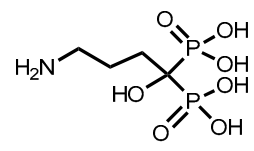

4

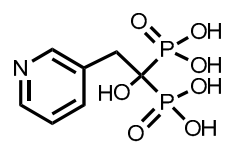

5

Figure 1. Chemical structures of pyrophosphoric acid, general formula of bisphosphonic acids and representative FDA-approved bisphosphonates clinically employed for the treatment of different bone disorders.

Pioneering work of Rogers et al. had shown that apoptosis induced by bisphosphonates in J774 macrophages was associated with the inhibition of post-translational prenylation of proteins such as Ras and that this effect can be reverted by the addition of components of the mevalonate pathway such as mevalonic acid, FPP, and GGPP [25]. Many years later, it was established that the primary molecular target of bisphosphonates was farnesyl diphosphate synthase (FPPS) [26]. Selective inhibition of parasite growth may be possible due to the incidence of very fast proliferation of parasites together with the presence of calcium polyphosphate stores in the acidocalcisomes, which mimic the calcium hydroxyapatite surfaces found in bone, facilitating bisphosphonate drug uptake $[27,28]$.

A relevant achievement of our laboratory was the finding that linear bisphosphonates became efficient antiparasitic agents [29-31] where, in most of them, the hydroxyl group at the C-1 position, usually found in those bisphosphonates currently employed in the treatment of bone disorders, is absent [26]. Compounds 6-9 were the first examples of linear bisphosphonates, which turned out to be antiparasitic agents (trypanosomatids and Apicomplexan parasites) targeting parasitic FPPS [7,29-32]. For example, 6 is a moderate growth inhibitor of intracellular T. cruzi [29] and also against tachyzoites of $T$. gondii [7,32], whereas 7 is effective against $P$. falciparum [7]. Compound 8 is practically devoid of antiparasitic activity [30], whereas 9 shows similar cellular activity against T. cruzi [31]. In addition, $\alpha$-fluoro-1,1-bisphosphonates $\mathbf{1 0}$ and $\mathbf{1 1}$ are neither effective against amastigotes of T. cruzi nor the target enzyme TcFPPS, but they are extremely potent inhibitors of the enzymatic activity of TgFPPS [33]. Of paramount concern are 2-alkyl(amino)ethyl-1,1-bisphosphonates, which are potent inhibitors of T. cruzi proliferation acting against the target enzyme (TcFPPS) at the low nanomolar range [34,35]. Undoubtedly, compounds 12-14 emerge as pertinent members of this type of bisphosphonates. For example, $\mathbf{1 2}$ is considerably more potent than the well-known antiparasitic agent WC-9 against T. cruzi (amastigotes) [34], under the same assays conditions [36]; while 13 is 
a very effective inhibitor of the enzymatic activities of TcFPPS and of TgFPPS [34]. Moreover, 14, the bisphosphonate bearing a long aliphatic chain, is an effective growth inhibitor of T. cruzi [35]. Compound $\mathbf{1 5}$ is an interesting example of linear bisphosphonates synthesized by an attempt to improve structures 12-14. Compound 15 had been designed based on the fact that the presence of electron withdrawing group (HO-) at C-1 would enhance the ability to coordinate $\mathrm{Mg}^{2+}$, would increase $\mathrm{p} K_{\mathrm{a}}$ and also by the fact that most bisphosphonates clinically in use have this functionality at C-1 [37]. Compound 15 is devoid of activity against T. cruzi growth and TcFPPS, but exhibited a potent and selective inhibition of the enzymatic activity towards TgFPPS [37]. Linear sulfur-containing bisphosphonates are interesting examples of selective anti-Toxoplasma agents as it is the case of $\mathbf{1 6}$ and 17 [38]. Certainly, $\mathbf{1 6}$ is a potent inhibitor of T. gondii proliferation. This cellular activity was associated with a potent action against the target enzyme TgFPPS [38], while $\mathbf{1 7}$ is an unusually potent inhibitor towards TgFPPS [38]. The methylsulfonium derivative 18 has proven to be a moderate growth inhibitor against both T. cruzi and T. gondii cells, but a very potent inhibitor of the enzymatic activity towards the target enzymes TcFPPS and TgFPPS (Figure 2; Table 1) [38].
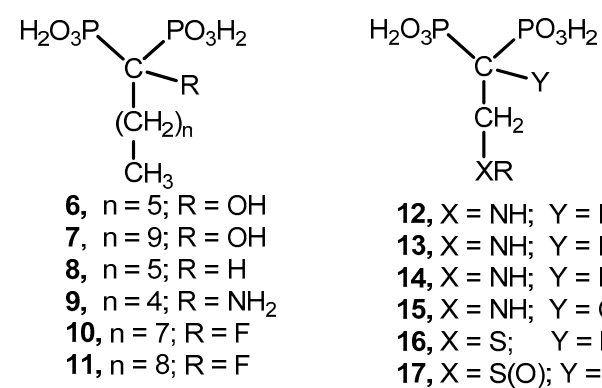

$$
\begin{aligned}
& \text { 12, } \mathrm{X}=\mathrm{NH} ; \mathrm{Y}=\mathrm{H} ; \mathrm{R}=n \text {-hexyl } \\
& 13, \mathrm{X}=\mathrm{NH} ; \mathrm{Y}=\mathrm{H} ; \mathrm{R}=n \text {-heptyl } \\
& \text { 14, } \mathrm{X}=\mathrm{NH} ; \mathrm{Y}=\mathrm{H} ; \mathrm{R}=n \text {-dodecyl } \\
& 15, \mathrm{X}=\mathrm{NH} ; \mathrm{Y}=\mathrm{OH} ; \mathrm{R}=n \text {-octyl } \\
& 16, \mathrm{X}=\mathrm{S} ; \mathrm{Y}=\mathrm{H} ; \mathrm{R}=n \text {-decyl } \\
& 17, \mathrm{X}=\mathrm{S}(\mathrm{O}) ; \mathrm{Y}=\mathrm{H} ; \mathrm{R}=n \text {-heptyl } \\
& 18, \mathrm{X}=\mathrm{S}+\left(\mathrm{CH}_{3}\right) ; \mathrm{Y}=\mathrm{H} ; \mathrm{R}=\text {-octyl }
\end{aligned}
$$

Figure 2. Chemical structures of representative lineal bisphosphonic acids as putative antiparasitic agents.

Table 1. Biological activity of bisphosphonates previously prepared in our laboratory against T. cruzi (amastigotes), T. gondii (tachyzoites), TcFPPS, and TgFPPS.

\begin{tabular}{ccccc}
\hline Compound & $\begin{array}{c}\text { T. cruzi Growth } \\
\text { ED }_{\mathbf{5 0}}(\boldsymbol{\mu M})\end{array}$ & $\begin{array}{c}\text { TcFPPS } \\
\mathbf{I C}_{\mathbf{5 0}}(\boldsymbol{\mu M})\end{array}$ & $\begin{array}{c}\text { T. gondii Growth } \\
\text { ED }_{\mathbf{5 0}}(\boldsymbol{\mu M})\end{array}$ & $\begin{array}{c}\text { TgFPPS } \\
\mathbf{I C}_{\mathbf{5 0}}(\boldsymbol{\mu M})\end{array}$ \\
\hline $\mathbf{1 0}$ & $>10.0$ & $>1.0$ & $2.67 \pm 11.93$ & 0.035 \\
$\mathbf{1 1}$ & $>10.0$ & $>1.0$ & $>10.0$ & 0.060 \\
$\mathbf{1 2}$ & 0.84 & 0.49 & 9.4 & 0.14 \\
$\mathbf{1 3}$ & 10.0 & 0.058 & $>50.0$ & 0.095 \\
$\mathbf{1 4}$ & 0.67 & 0.81 & 6.23 & 0.093 \\
$\mathbf{1 5}$ & $>10.0$ & $>10.0$ & 2.0 & 0.039 \\
$\mathbf{1 6}$ & $>20.0$ & 0.097 & 1.8 & 0.021 \\
$\mathbf{1 7}$ & $>20.0$ & $>10.0$ & 5.0 & 0.009 \\
$\mathbf{1 8}$ & $>20.0$ & 0.040 & 7.0 & 0.013 \\
\hline
\end{tabular}

\section{Rationale}

Prospects in antiparasitic (antileishmanial, antitrypanosomal, and Apicomplexan) drug research have changed substantially since the availability of the crystal structure of several complexes of 2-alkylaminoethyl-1,1-bisphosphonates with T. cruzi farnesyl diphosphate synthase [39]. These X-ray crystallographic studies on the interaction of inhibitor $\mathbf{1 3}$ with TcFPPS $(\mathbf{1 3}-$ TcFPPS $[39,40]$ have indicated that the nitrogen atom does not coordinate the $\mathrm{Mg}^{2+}$ present at the active site of the target enzyme [40]. The tridentate coordination structure is circumvented to the hydroxyl groups bonded to the phosphorus atoms either for 2-alkylaminoethyl- or 1-hydroxy-1,1-bisphosphonates [41,42]. X-ray structures and thermodynamic data of the complexes TcFPPS with 12 and 13 indicated that these compounds bind to the allylic site of TcFPPS having the hydrophobic alkyl chain buried in 
the cavity that binds the isoprenoid chain of the substrate [39]. The structures of 19 and 20 were envisioned by molecular modeling studies, which suggested that these compounds would increase affinity substantially (Figure 3) [39].<smiles>C=C(C)CNCC(P)P(O)O</smiles>

19<smiles>CC(C)=CC/C=C(\C)CNCC(P(=O)(O)O)P(=O)(O)O</smiles>

20

Figure 3. Chemical structures of the designed target molecules based on crystallographic and molecular modeling studies.

On the other hand, $\alpha$-fluoro-2-alkylaminoethyl-1,1-bisphosphonates such as 54-61 are also very interesting structural variations taking into account the selective and potent action against TgFPPS exhibited by 1-fluoro-1,1-bisphosphonates [33] and also the selective and efficient biological activity exhibited by 2-alkylaminoethyl-1,1-bisphosphonates towards both T. cruzi cells and TcFPPS [34,35,39].

\section{Results and Discussion}

Preparation of the allylamine analog of the lead chemical structures 12-14 (compound 19) was performed according to already published methods [34,35]. Succinctly, the Michael-type acceptor 21 [43], which was synthesized from commercially available tetraethyl methylene-1,1-bisphosphonate, was reacted with 2-methylallylamine in methylene chloride to produce the Michael adduct 22. This compound was hydrolyzed via the method developed by McKenna [44,45] by treatment with bromotrimethylsilane in methylene chloride followed by digestion with methanol [38] to yield the desired compound 19 (Scheme 1). The synthesis of the desired compound 20 was attempted following a slightly modified method previously described by Mori and Ueda (Scheme 1) [46]. Therefore, methallyl chloride (23) was coupled with 2-mercaptopyridine (24) to give the corresponding sulfide 25 in $84 \%$ yield, which on reaction with $n$-buthyllithium and isopentenyl bromide at low temperature gave 27 in $45 \%$ yield. This compound was treated with $m$-chloroperbenzoic acid at $-20{ }^{\circ} \mathrm{C}$ to produce the corresponding sulfoxide $\mathbf{2 8}$ as main product and a small amount of the sulfone $\mathbf{2 9}$. Basic treatment of 28 with diethylamine gave rise to the rearranged allyl alcohol 30, which under Mitsunobu-type conditions by treatment with triphenylphosphine, diisopropyl azodicarboxylate and phtalimide [47] yielded amine precursor 31. Cleavage of the phtalimide protecting group by treatment with hidrazine in refluxing ethanol [47] gave rise to the desired amine 32 as a main product and the hydrated product 33 as a consequence of the reaction work-up. This compound was reacted with the Michael acceptor 21 to produce 34 in good yields. Unexpectedly, it was not possible to carry out deprotection of the tetraethyl ester functionality. The presence of two double bonds indicated that hydrolysis by treatment with concentrated hydrochloric acid was an unlikely transformation [43]. The most commonly employed deprotection protocol, that is, reaction with bromotrimethylsilane followed by digestion with methanol [43], underwent a retro-Michael reaction yielding the corresponding free amine 32 together with the Michael acceptor 21. Actually, this retro-Michael reaction could take place during or after silylation; therefore, it is more likely that 21 or its Michael adduct with amine (34) upon solvolysis there will probably be no silyl ester, just free acid, product of solvolysis of silyl ester (after/before Michael addition). Hydrolysis in the presence of 2,4,6-collidine as described for other closely related compounds [48] was not satisfactory to avoid this behavior. The silyl ester of 21 has been described as a suitable Michael acceptor for a number of soft nucleophiles [49,50]. However, in our hands, we were not able to prepare addition product 20 by reaction between the silyl ester of $\mathbf{2 1}$ and the allylic amine 32. Evidently, the presence of the amino group at the C-3 position is not compatible with the available methodology to perform this transformation and new chemistry has to be developed to encourage this task. Efforts in these aspects are currently being pursued in our laboratory. Synthetic efforts to prepare the sulfur-containing analog of the envisioned compound 20, namely, 35 are outlined in Scheme 1 starting form allylic alcohol 30. Thus, 30 was treated under Mitsunobu-type conditions with triphenylphosphine, diisopropyl azodicarboxylate and thioacetic 
acid [51] to yield the thiolacetate 36 , which was hydrolyzed by treatment with potassium carbonate [51] to produce the corresponding mercaptan 37 in low but reproducible yields. Michael addition of $\mathbf{3 7}$ on 21 gave rise to 38, which could not be converted into the envisioned title compound 35 .

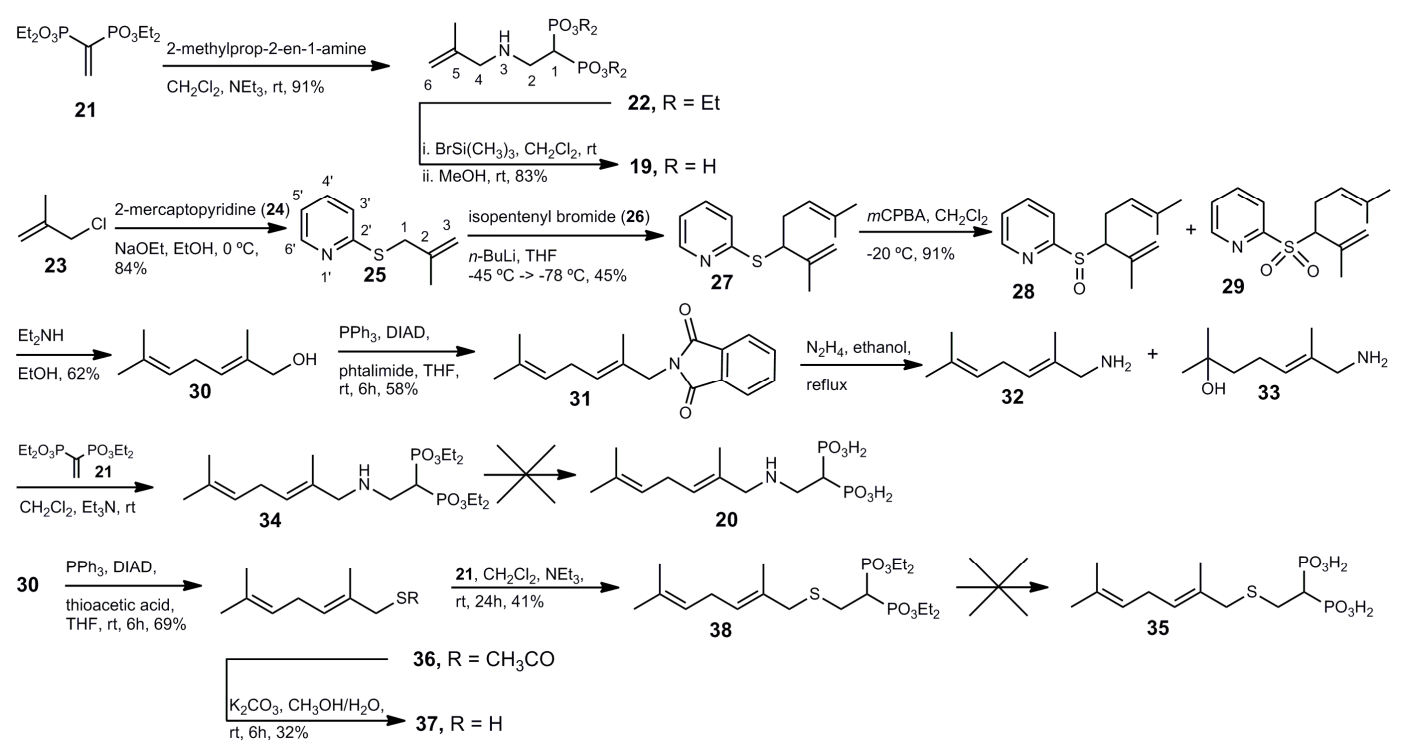

Scheme 1. Synthetic approach to access the envisioned putative inhibitors of farnesyl pyrophosphate synthase.

Compounds 39, 44 and 45 are isoprenoid derivatives, which are structurally related to 19,20 , and 35. These compounds, envisioned as optimized inhibitors of the target enzyme TcFPPS, are outlined in Scheme 2. Then, following a similar approach, geraniol (40) was converted into 41 by treatment with triphenylphosphine, diisopropyl azodicarboxylate and phtalimide in 80\% yield [51]. Synthesis of the free amine $\mathbf{4 2}$ followed by conjugate addition on $\mathbf{2 1}$ gave $\mathbf{4 3}$ in very good yields. Neither $\mathbf{4 3}$ nor its acetyl derivative $\mathbf{4 6}$ was able to produce the desired hypothetical desired compound 39 employing the currently known methods of phosphonates ester hydrolysis. Certainly, phosphonate esters bearing an amino group at the $\mathrm{C}-3$ position requires a new method for hydrolysis reaction to occur.

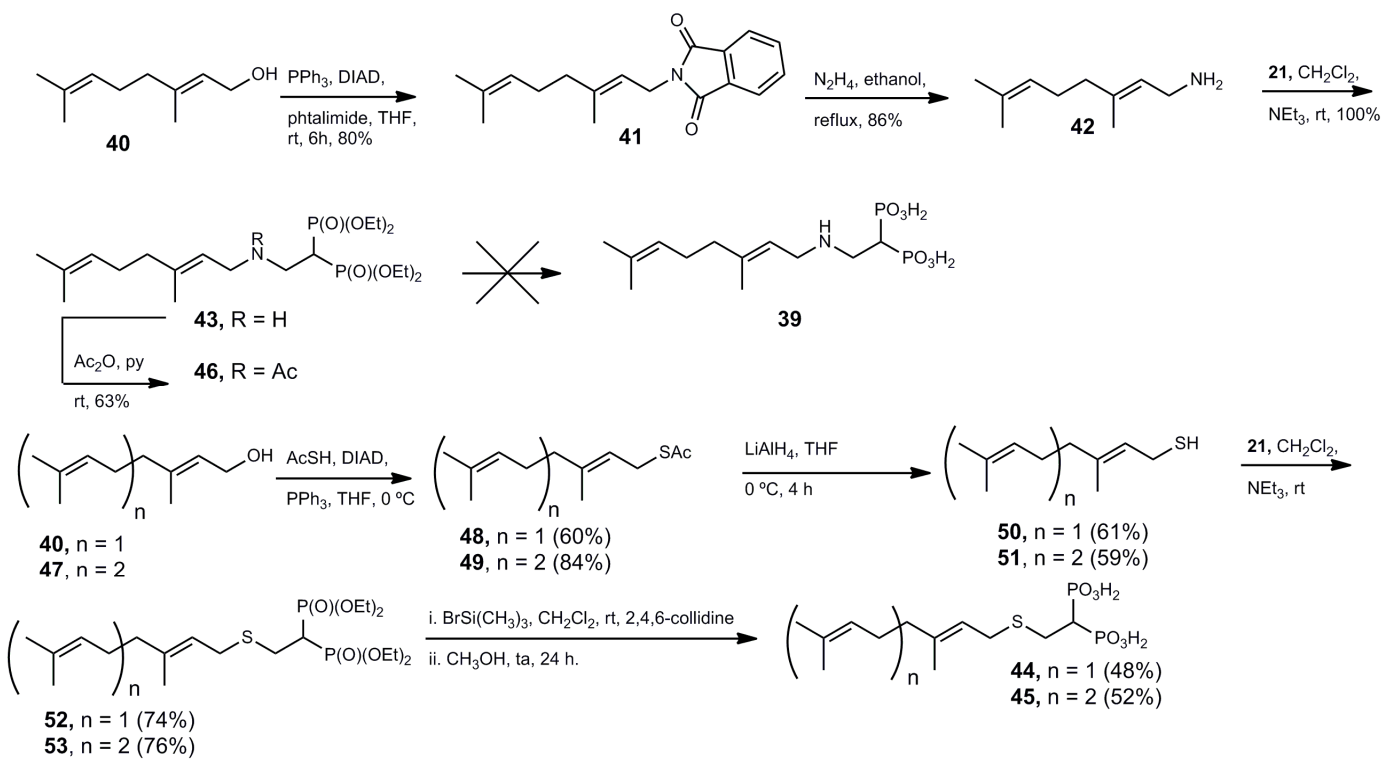

Scheme 2. Synthetic approach for the preparation of geranyl and farnesyl containing 1,1-bisphosphonates. 
On the other hand, 44 and 45 were straightforwardly prepared starting from geraniol (40) and farnesol (47), respectively. These alcohols under Mitsunobu type conditions [47] gave thiol acetates 48 and 49 in good yields that on treatment with lithium aluminum hydride produced the corresponding mercaptans 50 and 51. On reaction with 21, both compounds were converted into precursors 52 and 53, which after final treatment of bromotrimethylsilane and digestion with methanol yielded the title compounds 44 and 45 .

The synthesis of 1-fluoro-2-alkylaminoethyl-1,1-bisphosphonate derivatives $\mathbf{5 4 - 6 1}$ was accomplished following a similar approach with other closely related compounds [33] employing Selectfluor (1-chloromethyl-4-fluoro-1,4-diazobicyclo[2.2.2]octane bis(tetrafluoroborate), F-TEDA BF 4 ) as fluorinating agent as shown in Scheme 3 [52,53]. The presence of the amino group interfered with Selectfluor; therefore, protection of this group was required for the fluorination reaction to occur. Then, each tetraethyl 2-alkylaminoethyl-1,1-bisphosphonate 12-14, 62-66 was reacted with acetic anhydride in pyridine to produce the corresponding amides 67-74, which treated with sodium hydride followed by addition of Selectfluor [54] yielded the respective monofluoro bisphosphonate derivatives 75-82. Hydrolysis of these tetraethyl esters by treatment with concentrated hydrochloric acid generated the title compounds 54-61 (Scheme 3).

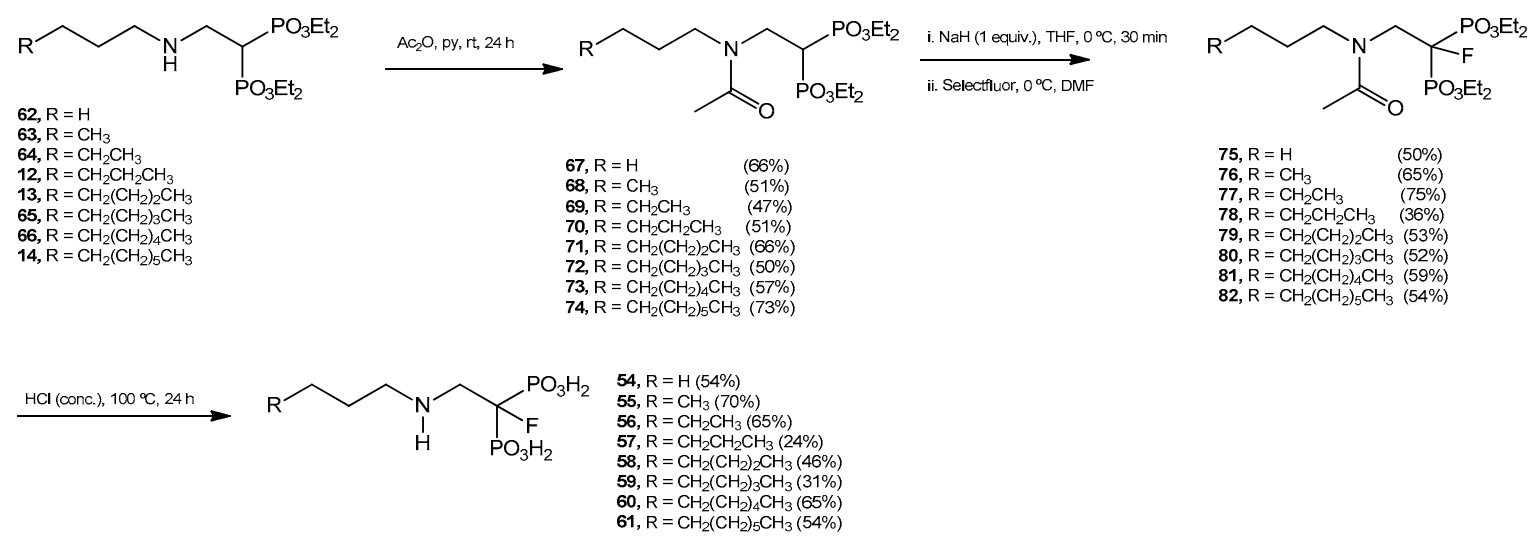

Scheme 3. Synthetic approach to access 2-alkylaminoethyl-1-fluoro-1,1-bisphosphonates.

Biological results are shown in Table 2. Most of the envisioned compounds resulted to be devoid of antiparasitic activity. Compound 19 was inactive against T. cruzi and T. gondii cells but exhibited a marginal activity against the target enzymes. Sulfur-containing isoprenoid 1,1-bisphosphonates 44 and 45 were also devoid of cellular activity against T. cruzi and T. gondii. Biological evaluation of $\alpha$-fluoro-2-alkyl(amino)ethyl-1,1-bisphosphonates 54-61 was very surprising. Certainly, in spite of having a small structural variation in comparison to 12-14, which are very effective growth inhibitors of T. cruzi cells targeting TcFPPS at the low nanomolar range, most of these fluorine derivatives, with the exception of 59, exhibited vanishing antiparasitic action. Bisphosphonate 59 was a growth inhibitor of tachyzoites of $T$. gondii at the low micromolar range $\left(\mathrm{ED}_{50}=4.0 \mu \mathrm{M}\right)$. It is worth mentioning the work on other bisphosphonates on bone mineral affinity and mevalonate pathway inhibition evaluations performed by McKenna et al. [55], where there is a drop of activity between $\alpha$-hydroxy and $\alpha$-fluoro analogs, but desoxy and fluoro analogs behave in a similar way. The derivatives that bear long chain length such as $\mathbf{6 0}$ and $\mathbf{6 1}$ turned out to be toxic compounds against Vero cells; therefore, they were not further analyzed.

In summary, the modest activity shown by these new families of bisphosphonates bring about important insights concerning structure-activity relationship. The simple replacement of the hydrogen atom by a fluorine atom had a dramatic effect on the biological activity, giving rise to inactive compounds. Effects in optimizing bisphosphonate structures are presently being pursued in our laboratory. 
Table 2. Biological activity of bisphosphonates against T. cruzi (amastigotes), T. gondii (tachyzoites), TcFPPS, TgFPPS, human FPPS (HhFPPS) and Vero cells $\ddagger$.

\begin{tabular}{|c|c|c|c|c|c|c|}
\hline Compound & $\begin{array}{l}\text { T. cruzi Growth } \\
\mathrm{ED}_{50}(\mu \mathrm{M})\end{array}$ & $\begin{array}{c}T c \text { FPPS } \\
\mathrm{IC}_{50}(\mu \mathrm{M})\end{array}$ & $\begin{array}{l}\text { T. gondii Growth } \\
\text { ED }_{50}(\mu \mathrm{M})\end{array}$ & $\begin{array}{c}T g \text { FPPS } \\
\mathrm{IC}_{50}(\mu \mathrm{M})\end{array}$ & $\begin{array}{c}H h \text { FPPS } \\
\mathrm{IC}_{50}(\mu \mathrm{M})\end{array}$ & $\begin{array}{c}\text { Cytotoxicity } \\
\mathrm{ED}_{50}(\mu \mathrm{M})\end{array}$ \\
\hline 19 & $>20$ & $4.5 \pm 2.8$ & $22.28 \pm 11.93$ & $0.183 \pm 0.063$ & $6.6 \pm 2.4$ & $>200$ \\
\hline 44 & $>10.0$ & NT & $>10.0$ & $0.313 \pm 0.027$ & NT & NT \\
\hline 54 & $>10.0$ & NT & NT & NT & NT & NT \\
\hline 55 & $>10.0$ & $0.097 \pm 0.020$ & $>10.0$ & $0.091 \pm 0.032$ & NT & $>200$ \\
\hline 56 & $>10.0$ & NT & $>10.0$ & NT & NT & NT \\
\hline 57 & $>10.0$ & NT & $>10.0$ & NT & NT & NT \\
\hline 60 & cytotoxic $†$ & NT & & NT & NT & 15.0 \\
\hline 61 & cytotoxic $\dagger$ & NT & & NT & NT & 20.0 \\
\hline benznidazole & $1.80 \pm 0.39$ & & & & & \\
\hline
\end{tabular}

$\ddagger$ Maximum concentration tested. NT $=$ not tested. $\uparrow$ visually.

\section{Experimental Section}

The glassware used in air- and/or moisture-sensitive reactions was flame-dried and reactions were carried out under dry argon. Unless otherwise noted, chemicals were commercially available and used without further purification. Solvents were distilled before use. Dichloromethane was distilled from phosphorus pentoxide. Nuclear magnetic resonance spectra were recorded with a Bruker AM-500 $\mathrm{MHz}$ spectrometer. The ${ }^{1} \mathrm{H}$ NMR spectra are referenced with respect to the residual $\mathrm{CHCl}_{3}$ proton of the solvent $\mathrm{CDCl}_{3}$ at $\delta=7.26 \mathrm{ppm}$. Coupling constants are reported in $\mathrm{Hz} .{ }^{13} \mathrm{C}$ NMR spectra were fully decoupled and are referenced to the middle peak of the solvent $\mathrm{CDCl}_{3}$ at $\delta=77.0 \mathrm{ppm}$. ${ }^{31} \mathrm{P} \mathrm{NMR}$ spectra are referenced with respect to the peak of $85 \% \mathrm{H}_{3} \mathrm{PO}_{4}$ as external reference. For comparative purposes, all NMR spectra acquired in $\mathrm{D}_{2} \mathrm{O}$ for free bisphosphonic acids were performed at the same conditions. Splitting patterns are designated as s, singlet; $d$, doublet; $t$, triplet; q, quadruplet; dd, double doublet, etc. Melting points were determined with a Fisher-Johns apparatus and are uncorrected. High-resolution mass spectra were obtained using a Bruker micrOTOF-Q II spectrometer, which is a hybrid quadrupole time of flight mass spectrometer with MS/MS capability. Analytical TLC was performed on commercial $0.2 \mathrm{~mm}$ aluminum-coated silica gel plates (F254) and visualized by $254 \mathrm{~nm} \mathrm{UV}$ or immersion in an aqueous solution of $\left(\mathrm{NH}_{4}\right)_{6} \mathrm{Mo}_{7} \mathrm{O}_{24} \cdot 4 \mathrm{H}_{2} \mathrm{O}(0.04 \mathrm{M}), \mathrm{Ce}\left(\mathrm{SO}_{4}\right)_{2}$ $(0.003 \mathrm{M})$ in concentrated $\mathrm{H}_{2} \mathrm{SO}_{4}(10 \%)$.

As judged from the homogeneity of the ${ }^{1} \mathrm{H},{ }^{13} \mathrm{C},{ }^{31} \mathrm{P}$ NMR spectra and HPLC analyses of the title compounds employing a Beckmann Ultrasphere ODS-2 column $5 \mu \mathrm{M}, 250 \times 10 \mathrm{~mm}$ eluting with water-acetonitrile $(9: 1)$ at $3.00 \mathrm{~mL} / \mathrm{min}$ with a refractive index detector indicated a purity $>97 \%$.

Tetraethyl 1-[(2-methylall-1-ylamino)ethyl] 1,1-bisphosphonate (22). A solution of compound 21 (300 mg, $1.0 \mathrm{mmol})$ in anhydrous methylene chloride $(10 \mathrm{~mL})$ was treated with triethylamine $(121.2 \mathrm{mg}, 168 \mu \mathrm{L}$, $1.2 \mathrm{mmol}$ ) and 2-methylallylamine hydrochloride (129 mg, $1.2 \mathrm{mmol})$ under an argon atmosphere. The reaction mixture was stirred at room temperature overnight. The solvent was evaporated and the residue was purified by column chromatography (silica gel) employing hexane-EtOAc (17:3) as eluent to produce $340 \mathrm{mg}$ (91\% yield) of 22 as a colorless oil: ${ }^{1} \mathrm{H}$ NMR $\left(500.13 \mathrm{MHz}, \mathrm{CDCl}_{3}\right) \delta 1.34$ $\left(\mathrm{t}, J=7.1 \mathrm{~Hz}, 12 \mathrm{H}, \mathrm{H}-2^{\prime}\right), 1.75\left(\mathrm{~s}, 3 \mathrm{H}, \mathrm{CH}_{3}\right.$ at C-5), $2.66(\mathrm{tt}, J=23.5,5.8 \mathrm{~Hz}, 1 \mathrm{H}, \mathrm{H}-1), 3.11(\mathrm{dt}, J=16.9$, $5.8 \mathrm{~Hz}, 2 \mathrm{H}, \mathrm{H}-2), 3.17$ (s, 2H, H-4), $4.19\left(\mathrm{~m}, 8 \mathrm{H}, \mathrm{H}-1^{\prime}\right), 4.83$ (br s, $\left.1 \mathrm{H}, \mathrm{H}-6_{\mathrm{a}}\right), 4.89$ (br s, $\left.1 \mathrm{H}, \mathrm{H}-6_{\mathrm{b}}\right) ;{ }^{13} \mathrm{C}$ NMR $\left(125.77 \mathrm{MHz}, \mathrm{CDCl}_{3}\right) \delta 16.4\left(\mathrm{dd}, J=6.4,2.6 \mathrm{~Hz}, \mathrm{C}-2^{\prime}\right), 20.6\left(\mathrm{CH}_{3}\right.$ at $\left.\mathrm{C}-5\right), 37.7(\mathrm{t}, J=132.5 \mathrm{~Hz}$, C-1), 44.9 (t, J = 4.3 Hz, C-2), $55.1(\mathrm{C}-4), 62.6\left(\mathrm{dd}, J=32.6,6.5 \mathrm{~Hz}, \mathrm{C}-1^{\prime}\right), 111.0$ (C-6) 143.3 (C-5); ${ }^{31} \mathrm{P}$ NMR (202.46 MHz, $\left.\mathrm{CDCl}_{3}\right) \delta$ 22.73. HRMS (ESI) calcd. for $\left(\mathrm{C}_{14} \mathrm{H}_{32} \mathrm{O}_{6} \mathrm{NP}_{2}\right)[\mathrm{M}+\mathrm{H}]^{+}$372.1707; found 372.1712 .

1-[(2-Methylall-1-ylamino)ethyl] 1,1-bisphosphonic Acid (19). To a solution of the tetraethyl ester 22 (320 $\mathrm{mg}, 0.86 \mathrm{mmol}$ ) in anhydrous methylene chloride was added dropwise trimethylsilyl bromide $(1.276 \mathrm{~g}, 1.10 \mathrm{~mL}, 8.6 \mathrm{mmol})$ under an argon atmosphere. The reaction mixture was stirred at room temperature for $48 \mathrm{~h}$. After cooling at $0{ }^{\circ} \mathrm{C}$, anhydrous methanol $(10 \mathrm{~mL})$ was added, and the resulting mixture was allowed to reach room temperature and stirred for $24 \mathrm{~h}$. The solution was 
then concentrated under reduced pressure. The residue was dissolved in dry methanol (10 mL) and subsequently concentrated under reduced pressure twice. The solvent was evaporated and the residue was crystallized from ethanol-water to yield $183 \mathrm{mg}\left(83 \%\right.$ yield) of pure 19 as a white solid: ${ }^{1} \mathrm{H}$ NMR $\left(500.13 \mathrm{MHz}, \mathrm{D}_{2} \mathrm{O}\right) \delta 1.74$ (s, 3H, $\mathrm{CH}_{3}$ at C-5), 2.44 (m, 1H, H-1), $\left.3.36 \mathrm{~m}, 2 \mathrm{H}, \mathrm{H}-2\right), 3.59$ (s, 2H, H-4), 5.03 $\left(\mathrm{s}, 1 \mathrm{H}, \mathrm{H}-6_{\mathrm{a}}\right), 5.12\left(\mathrm{~s}, 1 \mathrm{H}, \mathrm{H}-6_{\mathrm{b}}\right) ;{ }^{13} \mathrm{C} \mathrm{NMR}\left(125.77 \mathrm{MHz}, \mathrm{D}_{2} \mathrm{O}\right) \delta 19.5\left(\mathrm{CH}_{3}\right.$ at C-5), $35.7(\mathrm{t}, J=128.8 \mathrm{~Hz}$,

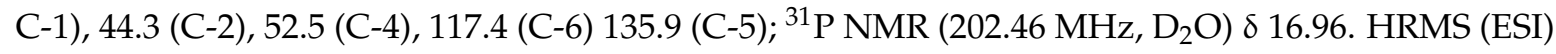
calcd. for $\left(\mathrm{C}_{6} \mathrm{H}_{15} \mathrm{O}_{6} \mathrm{NP}_{2} \mathrm{Na}\right)[\mathrm{M}+\mathrm{Na}]^{+} 282.0272$; found 282.0268 .

2-((2-Methylallyl)thio)pyridine (25). To a solution of sodium ethoxide (prepared from sodium $(620 \mathrm{mg}$, $27.0 \mathrm{mmol})$ and anhydrous ethanol $(20 \mathrm{~mL}))$ was added 2-mercaptopyridine $(24 ; 3.00 \mathrm{~g}, 27.0 \mathrm{mmol})$ at $0{ }^{\circ} \mathrm{C}$. The mixture was stirred for $15 \mathrm{~min}$. Then, methallyl chloride $(23 ; 3.06 \mathrm{~mL}, 2.81 \mathrm{~g}, 31.0 \mathrm{mmol})$ was added and the mixture was stirred at $0{ }^{\circ} \mathrm{C}$ for $3 \mathrm{~h}$. The solvent was evaporated and the residue was partitioned between water $(50 \mathrm{~mL})$ and ethyl ether $(50 \mathrm{~mL})$. The organic phase was washed with an aqueous $5 \%$ solution of sodium hydroxide $(2 \times 30 \mathrm{~mL})$ and water $(2 \times 30 \mathrm{~mL})$. The organic layer was dried $\left(\mathrm{MgSO}_{4}\right)$ and the solvent was evaporated to produce $3.743 \mathrm{~g}$ (84\% yield) of 25 as a colorless oil: ${ }^{1} \mathrm{H}$ NMR (500.13 MHz, $\left.\mathrm{CDCl}_{3}\right) \delta 1.85\left(\mathrm{~s}, 3 \mathrm{H}, \mathrm{CH}_{3}\right.$ at C-2), $3.86(\mathrm{~s}, 2 \mathrm{H}, \mathrm{H}-1), 4.86(\mathrm{t}, J=1.4 \mathrm{~Hz}, 1 \mathrm{H}$, H-3a) $5.02\left(\mathrm{q}, J=0.9 \mathrm{~Hz}, 1 \mathrm{H}, \mathrm{H}-3_{\mathrm{b}}\right), 6.97\left(\mathrm{ddd}, J=7.3,4.9,1.0 \mathrm{~Hz}, 1 \mathrm{H}, \mathrm{H}-5^{\prime}\right), 7.18(\mathrm{dt}, J=8.1,0.9 \mathrm{~Hz}$, $\left.1 \mathrm{H}, \mathrm{H}-3^{\prime}\right), 7.47\left(\mathrm{ddd}, J=7.9,7.5,1.8 \mathrm{~Hz}, 1 \mathrm{H}, \mathrm{H}-4^{\prime}\right), 8.42\left(\mathrm{ddd}, J=4.9,1.7,0.9 \mathrm{~Hz}, 1 \mathrm{H}, \mathrm{H}-6^{\prime}\right)$; ${ }^{13} \mathrm{C} \mathrm{NMR}$ (125.77 MHz, $\left.\mathrm{CDCl}_{3}\right) \delta 21.5\left(\mathrm{CH}_{3}\right.$ at C-2), 37.1 (C-3), $113.7(\mathrm{C}-1), 119.5\left(\mathrm{C}-5^{\prime}\right), 122.3\left(\mathrm{C}-3^{\prime}\right), 135.9\left(\mathrm{C}-4^{\prime}\right)$, $141.3(\mathrm{C}-2), 149.4\left(\mathrm{C}-6^{\prime}\right), 159.0\left(\mathrm{C}-2^{\prime}\right)$.

2-((2,6-Dimethylhepta-1,5-dien-3-yl)thio)pyridine (27). A solution of $\mathbf{2 5}$ (3.74 g, $22.7 \mathrm{mmol})$ in anhydrous tetrahydrofuran $(50 \mathrm{~mL})$ cooled at $-50{ }^{\circ} \mathrm{C}$ was treated dropwise with an $1.1 \mathrm{M}$ solution of $n$-butyllithium in hexane $(22 \mathrm{~mL})$. The reaction mixture turned deep-red and was cooled to $-78{ }^{\circ} \mathrm{C}$. Then, to the resulting mixture a solution of isopentenyl bromide $(26 ; 2.8 \mathrm{~mL}, 3.61 \mathrm{~g}, 24.2 \mathrm{mmol}) \mathrm{in}$ anhydrous tetrahydrofuran $(10 \mathrm{~mL})$ was added dropwise. The reaction mixture was stirred at $-78^{\circ} \mathrm{C}$ for $1 \mathrm{~h}$. Then, the mixture was allowed to reach room temperature gradually. The reaction was quenched by addition of a saturated solution of ammonium chloride $(50 \mathrm{~mL})$. The organic layer was separated and the aqueous phase was extracted with ethyl ether $(50 \mathrm{~mL})$. The combined organic layers were dried $\left(\mathrm{MgSO}_{4}\right)$ and the solvent was evaporated to give $2.384 \mathrm{~g}$ of 27 (45\% yield) as a colorless oil: ${ }^{1} \mathrm{H}$ NMR (500.13 MHz, $\left.\mathrm{CDCl}_{3}\right) \delta 1.64\left(\mathrm{~s}, 3 \mathrm{H}, \mathrm{CH}_{3}\right.$ at C-6), 1.68 (s, 3H, H-7), $1.82\left(\mathrm{~s}, 3 \mathrm{H}, \mathrm{CH}_{3}\right.$ at C-2), $2.43\left(\mathrm{~m}, 1 \mathrm{H}, \mathrm{H}-4_{\mathrm{a}}\right), 2.56\left(\mathrm{~m}, 1 \mathrm{H}, \mathrm{H}-4_{\mathrm{b}}\right), 4.40(\mathrm{dd}, J=8.6,6.6 \mathrm{~Hz}, 1 \mathrm{H}, \mathrm{H}-3), 4.85$ (br s, 1H, H-1 $\left.\mathrm{a}_{\mathrm{a}}\right), 4.98$ (br s, $\left.1 \mathrm{H}, \mathrm{H}-1_{\mathrm{b}}\right), 5.15$ (tt, $\left.J=7.0,1.2 \mathrm{~Hz}, 1 \mathrm{H}, \mathrm{H}-5\right), 6.92\left(\mathrm{ddt}, J=7.3,5.1,1.0 \mathrm{~Hz}, 1 \mathrm{H}, \mathrm{H}-5^{\prime}\right), 7.17$ (ddd, $\left.J=8.0,1.9,1.0 \mathrm{~Hz}, 1 \mathrm{H}, \mathrm{H}-3^{\prime}\right), 7.46\left(\mathrm{~m}, 1 \mathrm{H}, \mathrm{H}-4^{\prime}\right), 8.42\left(\mathrm{ddd}, J=4.0,1.8,0.9 \mathrm{~Hz}, 1 \mathrm{H}, \mathrm{H}-6^{\prime}\right) ;{ }^{13} \mathrm{C} \mathrm{NMR}$ $\left(125.77 \mathrm{MHz}, \mathrm{CDCl}_{3}\right) \delta 18.0\left(\mathrm{CH}_{3}\right.$ at $\left.\mathrm{C}-6\right), 19.5\left(\mathrm{CH}_{3}\right.$ at C-2), $25.8(\mathrm{C}-7), 32.1(\mathrm{C}-4), 51.2(\mathrm{C}-3), 113.3$ $(\mathrm{C}-1), 119.6\left(\mathrm{C}-5^{\prime}\right), 121.2\left(\mathrm{C}-3^{\prime}\right), 123.2(\mathrm{C}-5), 133.5(\mathrm{C}-6), 135.9\left(\mathrm{C}-4^{\prime}\right), 144.2(\mathrm{C}-2), 149.4\left(\mathrm{C}-6^{\prime}\right), 159.1\left(\mathrm{C}-2^{\prime}\right)$. HRMS (ESI) calcd. for $\mathrm{C}_{14} \mathrm{H}_{20} \mathrm{NS}[\mathrm{M}+\mathrm{H}]^{+}$234.1316; found 234.1319.

2-((2,6-Dimethylhepta-1,5-dien-3-yl)sulfinyl)pyridine (28) and 2-((2,6-dimethylhepta-1,5-dien-3-yl)sulfonyl) pyridine (29). To a solution of $27(5.91 \mathrm{mg}, 25.3 \mathrm{mmol})$ in methylene chloride $(100 \mathrm{~mL})$ cooled at $-25^{\circ} \mathrm{C}$ under an argon atmosphere was added dropwise a solution of $m$-chloroperbenzoic acid (77\% purity, $5.61 \mathrm{~g}, 26.6 \mathrm{mmol})$ in methylene chloride $(100 \mathrm{~mL})$. The mixture was stirred at $-25^{\circ} \mathrm{C}$ for $1 \mathrm{~h}$. Then, the temperature was allowed to reach room temperature. Then, the mixture was extracted with a saturated solution of potassium carbonate $(2 \times 75 \mathrm{~mL})$ and water $(2 \times 75 \mathrm{~mL})$. The organic phase was dried $\left(\mathrm{MgSO}_{4}\right)$, and the solvent was evaporated. The residue was purified by column chromatography (silica gel) eluting was hexane-EtOAc (19.1) to give $5.68 \mathrm{~g}$ of $\mathbf{2 8}$ (91\% yield) and $403 \mathrm{mg}$ of 29 (6\% yield) as colorless oils: Compound 28 (two diastereomers): ${ }^{1} \mathrm{H} \mathrm{NMR}\left(500.13 \mathrm{MHz}, \mathrm{CDCl}_{3}\right) \delta 1.42\left(\mathrm{~s}, 3 \mathrm{H}, \mathrm{CH}_{3}\right.$ at C-6), $1.55\left(\mathrm{~s}, 3 \mathrm{H}, \mathrm{CH}_{3}{ }^{*}\right.$ at C-6), $1.50(\mathrm{~s}, 3 \mathrm{H}, \mathrm{H}-7), 1.70\left(\mathrm{~s}, 3 \mathrm{H}, \mathrm{H}-7^{*}\right), 1.73\left(\mathrm{~s}, 3 \mathrm{H}, \mathrm{CH}_{3}\right.$ at C-2), 1.88 $\left(\mathrm{s}, 3 \mathrm{H}, \mathrm{CH}_{3}{ }^{*}\right.$ at C-2), $2.25\left(\mathrm{~m}, 1 \mathrm{H}, \mathrm{H}-4_{\mathrm{a}}\right), 2.60\left(\mathrm{~m}, 1 \mathrm{H}, \mathrm{H}-4_{\mathrm{a}}{ }^{*}\right), 2.47\left(\mathrm{~m}, 1 \mathrm{H}, \mathrm{H}-4_{\mathrm{b}}\right), 2.85\left(\mathrm{~m}, 1 \mathrm{H}, \mathrm{H}-4_{\mathrm{b}}{ }^{*}\right)$, $3.46(\mathrm{dd}, J=8.2,7.9 \mathrm{~Hz}, 1 \mathrm{H}, \mathrm{H}-3), 3.57$ (dd, $\left.J=10.3,4.9 \mathrm{~Hz}, 1 \mathrm{H}, \mathrm{H}-3^{*}\right), 4.48$ (br s, 1H, H-1 $\left.{ }_{\mathrm{a}}\right), 4.77$ $\left(\mathrm{p}, J=1.5 \mathrm{~Hz}, 1 \mathrm{H}, \mathrm{H}-1_{\mathrm{a}}{ }^{*}\right), 4.83$ (tp $\left., J=7.0,1.4 \mathrm{~Hz}, 1 \mathrm{H}, \mathrm{H}-3\right), 5.03$ (br s, 1H, H-1 $), 5.13(\mathrm{p}, J=1.5 \mathrm{~Hz}, 1 \mathrm{H}$, $\left.\mathrm{H}-1_{\mathrm{b}}{ }^{*}\right), 5.19\left(\mathrm{~m}, 1 \mathrm{H}, \mathrm{H}-3^{*}\right), 7.31$ (m, 1H, aromatic proton), 7.88-7.97 (m, 2H, aromatic proton), 8.60-8.64 (m, $1 \mathrm{H}$, aromatic proton); compound $29{ }^{1} \mathrm{H}$ NMR $\left(500.13 \mathrm{MHz}, \mathrm{CDCl}_{3}\right) \delta 1.57\left(\mathrm{~s}, 3 \mathrm{H}, \mathrm{CH}_{3}\right.$ at C-6), 1.67 
(s, 3H, H-7), $1.82\left(\right.$ br s, 3H, $\mathrm{CH}_{3}$ at C-2), $2.48\left(\mathrm{~m}, 1 \mathrm{H}, \mathrm{H}-4_{\mathrm{a}}\right), 2.72\left(\mathrm{~m}, 1 \mathrm{H}, \mathrm{H}-4_{\mathrm{b}}\right), 4.21(\mathrm{dd}, J=11.2,4.2 \mathrm{~Hz}$, 1H, H-3), 4.84 (br s, 1H, H-1 a), 4.88 (t, $J=7.0 \mathrm{~Hz}, 1 \mathrm{H}, \mathrm{H}-5), 5.02$ (br s, 1H, H-1 $), 7.51$ (ddd, $J=7.7,4.7$, $\left.1.1 \mathrm{~Hz}, 1 \mathrm{H}, \mathrm{H}-5^{\prime}\right), 7.91\left(\mathrm{dt}, J=7.7,1.7 \mathrm{~Hz}, 1 \mathrm{H}, \mathrm{H}-4^{\prime}\right), 8.02\left(\mathrm{~d}, J=7.9 \mathrm{~Hz}, 1 \mathrm{H}, \mathrm{H}-3^{\prime}\right), 8.74(\mathrm{ddd}, J=4.6,1.6$, $\left.0.8 \mathrm{~Hz}, 1 \mathrm{H}, \mathrm{H}-6^{\prime}\right) ;{ }^{13} \mathrm{C} \mathrm{NMR}\left(125.77 \mathrm{MHz}, \mathrm{CDCl}_{3}\right) \delta 17.9\left(\mathrm{CH}_{3}\right.$ at C-6), $20.2\left(\mathrm{CH}_{3}\right.$ at C-2), $24.6(\mathrm{C}-7), 25.7$ (C-4), 67.7 (C-3), $118.4(\mathrm{C}-1), 120.8\left(\mathrm{C}^{\prime} 5^{\prime}\right), 123.4\left(\mathrm{C}-3^{\prime}\right), 127.1(\mathrm{C}-5), 135.1$ (C-6), $136.4(\mathrm{C}-2), 137.7\left(\mathrm{C}-4^{\prime}\right)$, $150.1\left(\mathrm{C}-6^{\prime}\right), 156.6\left(\mathrm{C}-2^{\prime}\right)$.

(E)-2,6-Dimethylhepta-2,5-dien-1-ol (30). A solution of 28 (5.65 g, $22.7 \mathrm{mmol})$ in methanol (100 mL) was treated with diethylamine $(100 \mathrm{~mL})$ and the resulting mixture was stirred at room temperature overnight. The solvent was evaporated and the residue was partitioned between ethyl ether $(100 \mathrm{~mL})$ and water $(100 \mathrm{~mL})$. The aqueous phase was extracted with ethyl ether $(2 \times 50 \mathrm{~mL})$. The combined organic layers were washed with $5 \%$ hydrochloric acid $(2 \times 30 \mathrm{~mL})$, a saturated solution of sodium bicarbonate $(2 \times 30 \mathrm{~mL})$, brine $(3 \times 30 \mathrm{~mL})$. The organic layer was dried $\left(\mathrm{MgSO}_{4}\right)$ and the solvent was evaporated. The product was purified by column chromatography (silica gel) eluting with a mixture of hexane EtOAc (19:1) to yield $1.973 \mathrm{~g}$ (62\% yield) of alcohol 30 as a colorless oil: $R_{\mathrm{f}}=0.51$ (hexane-EtOAc, 1:1): ${ }^{1} \mathrm{H} \mathrm{NMR}\left(500.13 \mathrm{MHz} \mathrm{CDCl}_{3}\right) \delta 1.64(\mathrm{~s}, 3 \mathrm{H}, \mathrm{C}-7), 1.70\left(\mathrm{~s}, 6 \mathrm{H}, \mathrm{CH}_{3}\right.$ at C-2, $\mathrm{CH}_{3}$ at C-6), $2.73(\mathrm{t}, J=7.2 \mathrm{~Hz}, 2 \mathrm{H}, \mathrm{H}-4), 4.00$ (s, 2H, H-1), 5.11 (tp, $J=7.0,1.3 \mathrm{~Hz}, 1 \mathrm{H}, \mathrm{H}-3), 5.39$ (tq, $J=7.3$, $1.3 \mathrm{~Hz}, 1 \mathrm{H}, \mathrm{H}-5) ;{ }^{13} \mathrm{C}$ NMR $\left(125.77 \mathrm{MHz}, \mathrm{CDCl}_{3}\right) \delta 13.6\left(\mathrm{CH}_{3}\right.$ at C-2), $17.7\left(\mathrm{CH}_{3}\right.$ at C-6), $25.7(\mathrm{C}-7), 26.7$ (C-4), 69.0 (C-1), 122.4 (C-3), 125.1 (C-5), 132.0 (C-6), 134.5 (C-2).

(E)-2-(2,6-Dimethylhepta-2,5-dien-1-yl)isoindoline-1,3-dione (31). To a mixture of alcohol 30 (406 mg, $2.9 \mathrm{mmol})$, triphenylphosphine $(911.3 \mathrm{mg}, 3.5 \mathrm{mmol})$, phtalimide $(511.2 \mathrm{mg}, 3.5 \mathrm{mmol})$ in anhydrous tetrahydrofuran $(10 \mathrm{~mL})$ cooled at $0{ }^{\circ} \mathrm{C}$ was added a solution of diisopropyl azodicarboxylate (702.6 mg, $3.5 \mathrm{mmol})$. The reaction mixture was stirred at room temperature overnight. The solvent was evaporated and the residue was purified by column chromatography (silica gel) eluting with a mixture of hexane-EtOAc (19:1) to give $453.0 \mathrm{mg}$ (58\% yield) of pure 31 as a colorless oil: ${ }^{1} \mathrm{H}$ NMR $\left(500.13 \mathrm{MHz} \mathrm{CDCl}_{3}\right) \delta 1.60(\mathrm{~s}, 3 \mathrm{H}, \mathrm{C}-7), 1.67\left(\mathrm{~s}, 6 \mathrm{H}, \mathrm{CH}_{3}\right.$ at $\mathrm{C}-2, \mathrm{CH}_{3}$ at C-6), $2.70(\mathrm{t}, J=7.3 \mathrm{~Hz}, 2 \mathrm{H}$, H-4), 4.20 (s, 2H, H-1), 5.05 (tp, $J=7.0,1.4 \mathrm{~Hz}, 1 \mathrm{H}, \mathrm{H}-3), 5.36$ (tq, $J=7.2,1.3 \mathrm{~Hz}, 1 \mathrm{H}, \mathrm{H}-5) ;{ }^{13} \mathrm{C} \mathrm{NMR}$ $\left(125.77 \mathrm{MHz}, \mathrm{CDCl}_{3}\right) \delta 14.6\left(\mathrm{CH}_{3}\right.$ at C-2), $17.7\left(\mathrm{CH}_{3}\right.$ at C-6), $25.6(\mathrm{C}-7), 26.9(\mathrm{C}-4), 45.0(\mathrm{C}-1), 122.3(\mathrm{C}-3)$, $123.3\left(\mathrm{C}-2^{\prime}\right), 126.6(\mathrm{C}-5), 129.0\left(\mathrm{C}-1^{\prime}\right), 132.0$ (C-6), 132.1 (C-2), 133.9 (C-3'), 168.3 (CO). HRMS (ESI) calcd. for $\mathrm{C}_{17} \mathrm{H}_{19} \mathrm{O}_{2} \mathrm{NNa}[\mathrm{M}+\mathrm{Na}]^{+}$292.1313; found 292.1315 .

(E)-2,6-Dimethylhepta-2,5-dien-1-amine (32) and (E)-7-amino-2,6-dimethylhept-5-en-2-ol (33). A solution of $31(400 \mathrm{mg}, 1.5 \mathrm{mmol})$ in ethanol $(10 \mathrm{~mL})$ was treated with a solution of $60 \%$ hidrazine in water $(0.1 \mathrm{~mL})$. The reaction mixture was refluxed for $3 \mathrm{~h}$. The mixture was allowed to cool to room temperature and was filtered. The solvent was evaporated. The residue was treated with a $20 \%$ aqueous solution of hydrochloric acid $(10 \mathrm{~mL})$ and the mixture was filtered. The $\mathrm{pH}$ of the solution was adjusted to 11 by addition of a $1.0 \mathrm{M}$ solution of sodium hydroxide. The aqueous solution was extracted with methylene chloride $(3 \times 30 \mathrm{~mL})$, dried $\left(\mathrm{MgSO}_{4}\right)$ and the solvent was evaporated. The residue was purified by column chromatography (silica gel) employing a mixture of hexane-EtOAc (4:1) to give $175.4 \mathrm{mg}$ of 32 (84\% yield) and $21.2 \mathrm{mg}$ of 33 (9\% yield): Compound 32 : ${ }^{1} \mathrm{H}$ NMR $\left(500.13 \mathrm{MHz}_{2} \mathrm{CDCl}_{3}\right) \delta 1.64(\mathrm{~s}, 3 \mathrm{H}, \mathrm{C}-7), 1.66\left(\mathrm{CH}_{3}\right.$ at C-2), $1.70\left(\mathrm{CH}_{3}\right.$ at C-6), $2.72(\mathrm{t}, J=7.1 \mathrm{~Hz}, 2 \mathrm{H}$, $\mathrm{H}-4), 3.17$ (s, 2H, H-1), 5.11 (tq, $J=7.1,1.2 \mathrm{~Hz}, 1 \mathrm{H}, \mathrm{H}-3), 5.26$ (tt, $J=7.1,1.0 \mathrm{~Hz}, 1 \mathrm{H}, \mathrm{H}-5) ;{ }^{13} \mathrm{C} \mathrm{NMR}$ $\left(125.77 \mathrm{MHz}, \mathrm{CDCl}_{3}\right) \delta 14.6\left(\mathrm{CH}_{3}\right.$ at C-2), $17.7\left(\mathrm{CH}_{3}\right.$ at C-6), $25.7(\mathrm{C}-7), 26.8(\mathrm{C}-4), 49.9(\mathrm{C}-1), 122.6(\mathrm{C}-3)$, 122.9 (C-5), 131.7 (C-6), 136.3 (C-2). Compound 33: ${ }^{1} \mathrm{H}$ NMR (500.13 MHz, CDCl 3 ) $\delta 1.24$ (s, 6H, H-7, $\mathrm{CH}_{3}$ at C-6), $1.36\left(\mathrm{~s}, 2 \mathrm{H}, \mathrm{NH}_{2}\right), 1.55(\mathrm{~m}, 2 \mathrm{H}, \mathrm{H}-5), 1.66\left(\mathrm{~s}, 3 \mathrm{H}, \mathrm{CH}_{3}\right.$ at C-2), $2.11(\mathrm{~m}, 2 \mathrm{H}, \mathrm{H}-4), 3.17(\mathrm{~s}, 2 \mathrm{H}$, $\mathrm{H}-1), 5.30$ (tq, $J=7.1,1.2 \mathrm{~Hz}, 1 \mathrm{H}, \mathrm{H}-3) ;{ }^{13} \mathrm{C} \mathrm{NMR}\left(125.77 \mathrm{MHz}, \mathrm{CDCl}_{3}\right) \delta 14.5\left(\mathrm{CH}_{3}\right.$ at C-2), $22.7(\mathrm{C}-4)$, $29.2\left(\mathrm{C}-7, \mathrm{CH}_{3}\right.$ at C-6), 43.5 (C-5), 49.9 (C-1), 70.8 (C-6), 123.7 (C-3), 136.6 (C-2). HRMS (ESI) calcd. for $\mathrm{C}_{9} \mathrm{H}_{19} \mathrm{ONNa}[\mathrm{M}+\mathrm{Na}]^{+}$180.1364; found 180.1353 .

Tetraethyl (E)-2,6-dimethylhepta-2,5-dien-1-ylamino ethyl-1,1-bisphosphonate (34). A solution of 21 (327.8 mg, $1.1 \mathrm{mmol})$ in anhydrous methylene chloride $(10 \mathrm{~mL})$ was treated with triethylamine $(133.1 \mathrm{mg}, 185 \mu \mathrm{L}, 1.3 \mathrm{mmol})$ and $32(152.0 \mathrm{mg}, 1.1 \mathrm{mmol})$ as described for the preparation of 22 . Evaporation of the solvent yielded $483.2 \mathrm{mg}$ (100\% yield) of pure 34 as a colorless oil: ${ }^{1} \mathrm{H}$ NMR 
$\left(500.13 \mathrm{MHz}, \mathrm{CDCl}_{3}\right) \delta 1.34\left(\mathrm{t}, J=7.1 \mathrm{~Hz}, 12 \mathrm{H}, \mathrm{CH}_{2} \mathrm{CH}_{3}\right), 1.63(\mathrm{~s}, 3 \mathrm{H}, \mathrm{C}-10), 1.66\left(\mathrm{CH}_{3}\right.$ at $\left.\mathrm{C}-5\right), 1.68$ $\left(\mathrm{CH}_{3}\right.$ at C-9), $2.64(\mathrm{tt}, J=23.7,5.9 \mathrm{~Hz}, 1 \mathrm{H}, \mathrm{H}-1), 2.71(\mathrm{t}, J=6.8 \mathrm{~Hz}, 2 \mathrm{H}, \mathrm{H}-7), 3.08(\mathrm{dt}, J=16.8,5.9 \mathrm{~Hz}$, 2H, H-2), $3.12(\mathrm{~s}, 2 \mathrm{H}, \mathrm{H}-4), 4.18\left(\mathrm{~m}, 8 \mathrm{H}, \mathrm{CH}_{2} \mathrm{CH}_{3}\right), 5.09(\mathrm{tt}, J=7.1,1.3 \mathrm{~Hz}, 1 \mathrm{H}, \mathrm{H}-6), 5.29(\mathrm{t}, J=7.2 \mathrm{~Hz}$, $1 \mathrm{H}, \mathrm{H}-8) ;{ }^{13} \mathrm{C}$ NMR $\left(125.77 \mathrm{MHz}, \mathrm{CDCl}_{3}\right) \delta 14.6\left(\mathrm{CH}_{3}\right.$ at C-5), $16.3\left(\mathrm{~d}, \mathrm{~J}=3.0 \mathrm{~Hz}, \mathrm{CH}_{2} \mathrm{CH}_{3}\right), 16.4$ $\left(\mathrm{d}, J=2.7 \mathrm{~Hz}, \mathrm{CH}_{2} \mathrm{C}^{\prime} \mathrm{H}_{3}\right), 18.7\left(\mathrm{CH}_{3}\right.$ at $\left.\mathrm{C}-9\right), 24.7(\mathrm{C}-10), 26.9(\mathrm{C}-7), 37.6(\mathrm{t}, J=132.4 \mathrm{~Hz}, \mathrm{C}-1), 44.8(\mathrm{t}$, $J=4.3 \mathrm{~Hz}, \mathrm{C}-2), 57.0(\mathrm{C}-4), 62.4\left(\mathrm{~d}, J=6.6 \mathrm{~Hz}, \mathrm{CH}_{2} \mathrm{CH}_{3}\right), 62.7\left(\mathrm{~d}, J=6.6 \mathrm{~Hz}, \mathrm{C}^{\prime} \mathrm{H}_{2} \mathrm{CH}_{3}\right), 122.8(\mathrm{C}-6)$, 125.1 (C-8), 131.5 (C-9), 132.8 (C-5); ${ }^{31}$ P NMR (202.46 MHz, CDCl ${ }_{3}$ ) $\delta 22.82$ ppm.

(E)-S-(2,6-Dimethylhepta-2,5-dien-1-yl) ethanethioate (36). To a mixture of alcohol $30(471.0 \mathrm{mg}$, $3.4 \mathrm{mmol})$, triphenylphosphine $(1.32 \mathrm{~g}, 5.04 \mathrm{mmol})$, thioacetic acid $(255.7 \mathrm{mg}, 3.4 \mathrm{mmol})$ in anhydrous tetrahydrofuran $(10 \mathrm{~mL})$ cooled at $0{ }^{\circ} \mathrm{C}$ was added a solution of diisopropyl azodicarboxylate $(1.02 \mathrm{~g}$, $5.04 \mathrm{mmol}$ ) as depicted for the preparation of 31. The product was purified by column chromatography (silica gel) eluting with a mixture of hexane-EtOAc (49:1) to give $459.0 \mathrm{mg}$ (69\% yield) of $\mathbf{3 6}$ as a colorless oil: $R_{\mathrm{f}} 0.37$ (hexane-EtOAc, 19:1); ${ }^{1} \mathrm{H}$ NMR $\left(500.13 \mathrm{MHz}, \mathrm{CDCl}_{3}\right) \delta 1.62(\mathrm{~s}, 3 \mathrm{H}, \mathrm{C}-7), 1.65$ $\left(\mathrm{m}, 3 \mathrm{H}, \mathrm{CH}_{3}\right.$ at C-2), $1.68\left(\mathrm{~d}, J=1.1 \mathrm{~Hz}, 3 \mathrm{H}, \mathrm{CH}_{3}\right.$ at $\left.\mathrm{C}-6\right), 2.33\left(\mathrm{~s}, 3 \mathrm{H}, \mathrm{CH}_{3} \mathrm{C}(\mathrm{O})\right), 2.68(\mathrm{t}, J=6.9 \mathrm{~Hz}$, $2 \mathrm{H}, \mathrm{H}-4), 3.55$ (q, $J=0.9 \mathrm{~Hz}, 2 \mathrm{H}, \mathrm{H}-1), 5.11(\mathrm{tq}, J=7.1,1.2 \mathrm{~Hz}, 1 \mathrm{H}, \mathrm{H}-3), 5.38(\mathrm{tq}, J=7.3,1.2 \mathrm{~Hz}, 1 \mathrm{H}$, $\mathrm{H}-5) ;{ }^{13} \mathrm{C}$ NMR $\left(125.77 \mathrm{MHz}, \mathrm{CDCl}_{3}\right) \delta 15.2\left(\mathrm{CH}_{3}\right.$ at C-2), $17.7\left(\mathrm{CH}_{3}\right.$ at C-6), 25.7 (C-7), $27.2(\mathrm{C}-4), 30.5$ $\left(\mathrm{CH}_{3} \mathrm{C}(\mathrm{O})\right), 38.2(\mathrm{C}-1), 122.2(\mathrm{C}-3), 127.9(\mathrm{C}-5), 130.0(\mathrm{C}-2), 132.1(\mathrm{C}-6), 195.1\left(\mathrm{CH}_{3} \mathrm{C}(\mathrm{O})\right)$.

(E)-2,6-Dimethylhepta-2,5-dien-1-thiol (37). To a solution of 36 (353.0 $\mathrm{mg}, 1.78 \mathrm{mmol})$ in $20.0 \mathrm{~mL}$ of a mixture of methanol-water (5:3) was added potassium carbonate $(100 \mathrm{mg}, 0.72 \mathrm{mmol})$. The reaction mixture was stirred at room temperature for $6 \mathrm{~h}$. Then, water $(50 \mathrm{~mL})$ was added and the mixture was extracted with methylene chloride $(3 \times 30 \mathrm{~mL})$. The combined organic layers were washed with brine $(2 \times 30 \mathrm{~mL})$, dried $\left(\mathrm{MgSO}_{4}\right)$ and the solvent was evaporated. The residue was purified by column chromatography (silica gel) eluting with hexane to yield $89 \mathrm{mg}$ ( $32 \%$ yield) of pure 37 as a colorless oil: $R_{\mathrm{f}} 0.57$ (hexane-EtOAc, 19:1); ${ }^{1} \mathrm{H}$ NMR $\left(300.18 \mathrm{MHz}, \mathrm{CDCl}_{3}\right) \delta 1.40(\mathrm{t}, J=7.8 \mathrm{~Hz}, 1 \mathrm{H}, \mathrm{SH}), 1.63(\mathrm{~s}, 3 \mathrm{H}$, C-7), $1.69\left(\mathrm{~s}, 3 \mathrm{H}, \mathrm{CH}_{3}\right.$ at C-2), $1.75\left(\mathrm{q}, J=1.0 \mathrm{~Hz}, 3 \mathrm{H}, \mathrm{CH}_{3}\right.$ at C-6), $2.71(\mathrm{q}, J=7.9 \mathrm{~Hz}, 2 \mathrm{H}, \mathrm{H}-4), 3.12$ (d, $J=7.8 \mathrm{~Hz}, 2 \mathrm{H}, \mathrm{H}-1), 5.08$ (tp, $J=7.1,1.4 \mathrm{~Hz}, 1 \mathrm{H}, \mathrm{H}-3), 5.31$ (tq, $J=7.2,1.0 \mathrm{~Hz}, 1 \mathrm{H}, \mathrm{H}-5)$.

Tetraethyl (E)-2,6-dimethylhepta-2,5-dien-1-ylthioethyl-1,1-bisphosphonate (38). A solution of 21 (430.3 mg, $1.43 \mathrm{mmol})$ in anhydrous methylene chloride $(15 \mathrm{~mL})$ was treated with triethylamine $(147.1 \mathrm{mg}$, $205 \mu \mathrm{L}, 1.43 \mathrm{mmol})$ and $37(224.1 \mathrm{mg}, 1.43 \mathrm{mmol})$ as described for the preparation of 22 . The solvent was evaporated and the residue was purified by column chromatography (silica gel) employing hexane-EtOAc (4:1) as eluent to give $268 \mathrm{mg}$ (41\% yield) of 38 as a colorless oil: ${ }^{1} \mathrm{H}$ NMR $(500.13 \mathrm{MHz}$, $\left.\mathrm{CDCl}_{3}\right) \delta 1.366\left(\mathrm{t}, J=7.0 \mathrm{~Hz}, 6 \mathrm{H}, \mathrm{CH}_{2} \mathrm{CH}_{3}\right), 1.374\left(\mathrm{t}, J=7.1 \mathrm{~Hz}, 6 \mathrm{H}, \mathrm{CH}_{2} \mathrm{CH}_{3}^{\prime}\right), 1.64(\mathrm{~s}, 3 \mathrm{H}, \mathrm{C}-10), 1.70$ $\left(\mathrm{s}, 3 \mathrm{H}, \mathrm{CH}_{3}\right.$ at C-5), $1.74\left(\mathrm{~m}, 3 \mathrm{H}, \mathrm{CH}_{3}\right.$ at $\left.\mathrm{C}-9\right), 2.61(\mathrm{tt}, J=23.8,6.2 \mathrm{~Hz}, 1 \mathrm{H}, \mathrm{H}-1), 2.74(\mathrm{t}, J=7.6 \mathrm{~Hz}$, $2 \mathrm{H}, \mathrm{H}-7), 2.94(\mathrm{dt}, J=16.2,6.2 \mathrm{~Hz}, 2 \mathrm{H}, \mathrm{H}-2), 3.15(\mathrm{~s}, 2 \mathrm{H}, \mathrm{H}-4), 4.21\left(\mathrm{~m}, 8 \mathrm{H}, \mathrm{CH}_{2} \mathrm{CH}_{3}\right), 5.11$ (tp, J = 7.0, $1.6 \mathrm{~Hz}, 1 \mathrm{H}, \mathrm{H}-6), 5.31(\mathrm{t}, J=7.1 \mathrm{~Hz}, 1 \mathrm{H}, \mathrm{H}-8) ;{ }^{13} \mathrm{C} \mathrm{NMR}\left(125.77 \mathrm{MHz}, \mathrm{CDCl}_{3}\right) \delta 14.9\left(\mathrm{CH}_{3}\right.$ at C-5), $16.2\left(\mathrm{t}, J=3.3 \mathrm{~Hz}, \mathrm{CH}_{2} \mathrm{CH}_{3}\right), 16.4\left(\mathrm{dd}, J=6.5,1.3 \mathrm{~Hz}, \mathrm{CH}_{2} \mathrm{C}^{\prime} \mathrm{H}_{3}\right), 17.7\left(\mathrm{CH}_{3}\right.$ at $\left.\mathrm{C}-9\right), 25.6(\mathrm{C}-10), 26.6$ $(\mathrm{t}, J=5.0 \mathrm{~Hz}, \mathrm{C}-2), 27.2(\mathrm{C}-7), 38.7(\mathrm{t}, J=131.7 \mathrm{~Hz}, \mathrm{C}-1), 42.4 .0(\mathrm{C}-4), 62.7\left(\mathrm{~d}, J=4.8 \mathrm{~Hz}, \mathrm{CH}_{2} \mathrm{CH}_{3}\right)$, $62.8\left(\mathrm{~d}, \mathrm{~J}=6.7 \mathrm{~Hz}, \mathrm{C}^{\prime} \mathrm{H}_{2} \mathrm{CH}_{3}\right), 122.2$ (C-6), 127.9 (C-8), 130.0 (C-4), 132.1 (C-9); ${ }^{31} \mathrm{P}$ NMR (202.47 MHz, $\left.\mathrm{CDCl}_{3}\right) \delta 21.77 \mathrm{ppm}$.

(E)-2-(3,7-Dimethylocta-2,6-dien-1-yl)isoindoline-1,3-dione (41). To a mixture of geraniol 40 (1.00 g, $6.5 \mathrm{mmol})$, triphenylphosphine $(1.70 \mathrm{~g}, 6.5 \mathrm{mmol})$, phtalimide $(953.3 \mathrm{mg}, 6.5 \mathrm{mmol})$ in anhydrous tetrahydrofuran $(15 \mathrm{~mL})$ cooled at $0{ }^{\circ} \mathrm{C}$ was added a solution of diisopropyl azodicarboxylate $(1.38 \mathrm{~g}$, $6.5 \mathrm{mmol}$ ) as described for the preparation of 31 . The residue was purified by column chromatography (silica gel) eluting with hexane-EtOAc (19:1) to give $1.47 \mathrm{~g}$ (80 yield) of pure $\mathbf{4 1}$ as a colorless oil: $R_{\mathrm{f}} 0.52$ (hexane-EtOAc, 4:1); ${ }^{1} \mathrm{H}$ NMR $\left(200 \mathrm{MHz}, \mathrm{CDCl}_{3}\right) \delta 1.56(\mathrm{~s}, 3 \mathrm{H}, \mathrm{H}-8), 1.63\left(\mathrm{~s}, 3 \mathrm{H}, \mathrm{CH}_{3}\right.$ at C-7), $1.82\left(\mathrm{~s}, 3 \mathrm{H}, \mathrm{CH}_{3}\right.$ at C-3), $2.00(\mathrm{~m}, 2 \mathrm{H}, \mathrm{H}-5), 2.08(\mathrm{p}, J=7.0 \mathrm{~Hz}, 2 \mathrm{H}, \mathrm{H}-4), 4.27(\mathrm{~d}, J=7.2 \mathrm{~Hz}, 2 \mathrm{H}, \mathrm{H}-1)$, $5.05(\mathrm{~m}, 1 \mathrm{H}, \mathrm{H}-6), 5.27$ (t, $J=7.4 \mathrm{~Hz}, 1 \mathrm{H}, \mathrm{H}-2)$.

(E)-3,7-Dimethylocta-2,6-dien-1-amine (42). A solution of 41 (900 $\mathrm{mg}, 3.2 \mathrm{mmol}$ ) in ethanol (13 mL) was treated with a solution of $60 \%$ hidrazine in water $(150 \mu \mathrm{L}, 101 \mathrm{mg}, 3.2 \mathrm{mmol})$. The reaction mixture 
was refluxed for $3 \mathrm{~h}$ and was treated as described for the preparation of 32 to yield $422 \mathrm{mg}$ (86\% yield) as a colorless oil: ${ }^{1} \mathrm{H}$ NMR $\left(500.13 \mathrm{MHz}, \mathrm{CDCl}_{3}\right) \delta 1.60(\mathrm{~s}, 3 \mathrm{H}, \mathrm{H}-8), 1.63\left(\mathrm{~s}, 3 \mathrm{H}, \mathrm{CH}_{3}\right.$ at C-7), 1.69 (s, $3 \mathrm{H}, \mathrm{CH}_{3}$ at C-3), 2.00 (m, 2H, H-5), 2.08 (p, J = $\left.7.0 \mathrm{~Hz}, 2 \mathrm{H}, \mathrm{H}-4\right), 3.27$ (d, J = 6.8 Hz, 2H, H-1), 5.10 $(\mathrm{tp}, J=6.7,1.3 \mathrm{~Hz}, 1 \mathrm{H}, \mathrm{H}-6), 5.26(\mathrm{tp}, J=6.9,1.2 \mathrm{~Hz}, 1 \mathrm{H}, \mathrm{H}-2) ;{ }^{13} \mathrm{C} \mathrm{NMR}\left(125.77 \mathrm{MHz}, \mathrm{CDCl}_{3}\right) \delta 16.1$ $\left(\mathrm{CH}_{3}\right.$ at $\left.\mathrm{C}-3\right), 17.7\left(\mathrm{CH}_{3}\right.$ at C-7), 25.7 (C-8), 26.5 (C-5), $39.5(\mathrm{C}-4), 39.6$ (C-1), $124.1(\mathrm{C}-2), 125.9(\mathrm{C}-6), 131.5$ (C-7), 136.3 (C-3).

Tetraethyl 1-[((E)-3,7-dimethylocta-2,6-diene-1-amino)ethyl] 1,1-bisphosphonate (43). A solution of 21 $(400.1 \mathrm{mg}, 1.33 \mathrm{mmol})$ in anhydrous methylene chloride $(10 \mathrm{~mL})$ was treated with amine 42 (204.0 $\mathrm{mg}, 1.33 \mathrm{mmol}$ ) as described for the preparation of 22. Evaporation of the solvent gave $611.1 \mathrm{mg}$ (100\% yield) of pure 43 as a colorless oil: ${ }^{1} \mathrm{H}$ NMR $\left(500.13 \mathrm{MHz}, \mathrm{CDCl}_{3}\right) \delta 1.34(\mathrm{t}, J=7.1 \mathrm{~Hz}, 12 \mathrm{H}$, $\left.\mathrm{CH}_{2} \mathrm{CH}_{3}\right), 1.63(\mathrm{~s}, 3 \mathrm{H}, \mathrm{H}-11), 1.66\left(\mathrm{~s}, 3 \mathrm{H}, \mathrm{CH}_{3}\right.$ at C-10), $1.68\left(\mathrm{~s}, 3 \mathrm{H}, \mathrm{CH}_{3}\right.$ at C-6), $2.71(\mathrm{t}, J=6.8 \mathrm{~Hz}, 4 \mathrm{H}$, H-7, H-8), 2.65 (tt, $J=23.7,5.9 \mathrm{~Hz}, 1 \mathrm{H}, \mathrm{H}-1), 3.08$ (dt, J = 16.8, 5.9 Hz, 2H, H-2), 3.13 (br s, 2H, H-4), 5.09 $(\mathrm{tt}, J=7.1,1.3 \mathrm{~Hz}, 1 \mathrm{H}, \mathrm{H}-9), 5.29(\mathrm{t}, J=7.2 \mathrm{~Hz}, 1 \mathrm{H}, \mathrm{H}-5) ;{ }^{13} \mathrm{C} \mathrm{NMR}\left(125.77 \mathrm{MHz}, \mathrm{CDCl}_{3}\right) \delta 14.6\left(\mathrm{CH}_{3}\right.$ at C-6), $16.3\left(\mathrm{~d}, \mathrm{~J}=3.0 \mathrm{~Hz}, \mathrm{CH}_{2} \mathrm{CH}_{3}\right), 16.4\left(\mathrm{~d}, \mathrm{~J}=2.7 \mathrm{~Hz}, \mathrm{CH}_{2} \mathrm{CH}_{3}{ }^{\prime}\right), 17.7\left(\mathrm{CH}_{3}\right.$ at C-10), $25.6(\mathrm{C}-11), 26.9$ (C-8), $37.6(\mathrm{t}, J=132.4 \mathrm{~Hz}, \mathrm{C}-1), 44.8(\mathrm{t}, J=4.3 \mathrm{~Hz}, \mathrm{C}-2), 57.0(\mathrm{C}-4), 62.4\left(\mathrm{~d}, J=6.8 \mathrm{~Hz}, \mathrm{CH}_{2} \mathrm{CH}_{3}\right), 62.7$ $\left(\mathrm{d}, J=6.8 \mathrm{~Hz}, \mathrm{CH}_{2} \mathrm{CH}_{3}{ }^{\prime}\right), 122.8$ (C-5), 125.1 (C-9), 131.5 (C-10), 132.8 (C-6); ${ }^{31} \mathrm{P}$ NMR (202.46 MHz, $\left.\mathrm{CDCl}_{3}\right) \delta 22.82 \mathrm{ppm}$.

Tetraethyl 1-[((E)-3,7-dimethylocta-2,6-diene-1-acetamido)ethyl] 1,1-bisphosphonate (46). A solution of 43 $(227 \mathrm{mg}, 0.5 \mathrm{mmol})$ in anhydrous pyridine $(3.0 \mathrm{~mL})$ was treated with acetic anhydride $(3.0 \mathrm{~mL})$ and the mixture was stirred at room temperature overnight. The solvent was evaporated and the residue was purified by column chromatography (silica gel) eluting with a mixture of $\mathrm{CH}_{2} \mathrm{Cl}_{2}-$ methanol (19:1) to give $312.2 \mathrm{mg}$ (63\% yield) of pure 46 as a colorless oil: ${ }^{1} \mathrm{H}$ NMR $\left(500.13 \mathrm{MHz}, \mathrm{CDCl}_{3}\right) \delta 1.34$ $\left(\mathrm{t}, J=7.0 \mathrm{~Hz}, 6 \mathrm{H}, \mathrm{CH}_{2} \mathrm{CH}_{3}\right), 1.35\left(\mathrm{t}, J=7.1 \mathrm{~Hz}, 6 \mathrm{H}, \mathrm{CH}_{2} \mathrm{CH}_{3}{ }^{\prime}\right), 1.61(\mathrm{~s}, 3 \mathrm{H}, \mathrm{H}-11), 1.68\left(\mathrm{~s}, 3 \mathrm{H}, \mathrm{CH}_{3}\right.$ at C-10), 1.69 (s, 3H, $\mathrm{CH}_{3}$ at C-6), 2.00-2.11 (m, 4H, H-7, H-8), $2.07\left(\mathrm{~s}, 3 \mathrm{H}, \mathrm{C}(\mathrm{O}) \mathrm{CH}_{3}\right), 3.38$ (tt, J = 22.7, $5.5 \mathrm{~Hz}, 1 \mathrm{H}, \mathrm{H}-1), 3.71$ (ddd, $J=14.7,11.1,7.4 \mathrm{~Hz}, 2 \mathrm{H}, \mathrm{H}-2), 4.04$ (d, J = 6.4 Hz, 2H, H-4), 4.18 (m, 8H, $\left.\mathrm{CH}_{2} \mathrm{CH}_{3}\right), 5.07$ (m, 2H, H-5, H-9); $\left.{ }^{13} \mathrm{C} \mathrm{NMR} \mathrm{(125.77} \mathrm{MHz,} \mathrm{CDCl}_{3}\right) \delta 16.3\left(\mathrm{CH}_{3}\right.$ at C-6), 16.34 $\left(\mathrm{d}, J=6.7 \mathrm{~Hz}, \mathrm{CH}_{2} \mathrm{CH}_{3}\right), 16.37\left(\mathrm{~d}, \mathrm{~J}=6.6 \mathrm{~Hz}, \mathrm{CH}_{2} \mathrm{CH}_{3}{ }^{\prime}\right), 17.7\left(\mathrm{CH}_{3}\right.$ at $\left.\mathrm{C}-10\right), 21.9\left(\mathrm{C}(\mathrm{O}) \mathrm{CH}_{3}\right), 25.7$ (C-11), $26.3(\mathrm{C}-8), 34.4(\mathrm{t}, J=129.9 \mathrm{~Hz}, \mathrm{C}-1), 39.5(\mathrm{C}-7), 44.4(\mathrm{t}, J=3.5 \mathrm{~Hz}, \mathrm{C}-2), 48.8(\mathrm{C}-4), 62.4(\mathrm{~d}$, $\left.J=6.8 \mathrm{~Hz}, \mathrm{CH}_{2} \mathrm{CH}_{3}\right), 62.7\left(\mathrm{~d}, J=6.8 \mathrm{~Hz}, \mathrm{CH}_{2} \mathrm{CH}_{3}{ }^{\prime}\right), 119.7(\mathrm{C}-5), 123.6$ (C-9), $131.9(\mathrm{C}-10), 139.4(\mathrm{C}-6)$, $171.2\left(\mathrm{C}(\mathrm{O}) \mathrm{CH}_{3}\right) ;{ }^{31} \mathrm{P} \mathrm{NMR}\left(202.46 \mathrm{MHz} \mathrm{CDCl}_{3}\right) \delta 21.49 \mathrm{ppm}$.

(E)-S-(3,7-Dimethylocta-2,6-dien-1-yl) ethanethioate (48). To a mixture of geraniol 40 (1.00 g, $6.5 \mathrm{mmol})$, triphenylphosphine $(2.55 \mathrm{~g}, 9.7 \mathrm{mmol})$, thioacetic acid $(0.55 \mathrm{~mL}, 592.2 \mathrm{mg}, 7.8 \mathrm{mmol})$ in anhydrous tetrahydrofuran $(20 \mathrm{~mL})$ cooled at $0{ }^{\circ} \mathrm{C}$ was added a solution of diisopropyl azodicarboxylate $(1.62 \mathrm{~mL}, 1.66 \mathrm{~g}, 9.7 \mathrm{mmol})$ as depicted for the preparation of 31. The product was purified by column chromatography (silica gel) eluting with hexane-EtOAc (9:1) to give $828.2 \mathrm{mg}$ (60\% yield) of 48 as a colorless oil: $R_{\mathrm{f}} 0.39$ (hexane-EtOAc, 4:1); ${ }^{1} \mathrm{H}$ NMR $\left(500.13 \mathrm{MHz}, \mathrm{CDCl}_{3}\right) \delta 1.59(\mathrm{~s}, 3 \mathrm{H}, \mathrm{H}-8)$, $1.68\left(\mathrm{~s}, 6 \mathrm{H}, \mathrm{CH}_{3}\right.$ at $\mathrm{C}-3, \mathrm{CH}_{3}$ at C-7), $2.00(\mathrm{~m}, 2 \mathrm{H}, \mathrm{H}-4), 2.06(\mathrm{~m}, 2 \mathrm{H}, \mathrm{H}-4), 2.32\left(\mathrm{~s}, 3 \mathrm{H}, \mathrm{SC}(\mathrm{O}) \mathrm{CH}_{3}\right), 3.54$ $(\mathrm{d}, J=7.9 \mathrm{~Hz}, 2 \mathrm{H}, \mathrm{H}-1), 5.06$ (tp, $J=6.8,1.4 \mathrm{~Hz}, 1 \mathrm{H}, \mathrm{H}-6), 5.21(\mathrm{tp}, J=7.9,1.3 \mathrm{~Hz}, 1 \mathrm{H}, \mathrm{H}-2) ;{ }^{13} \mathrm{C} \mathrm{NMR}$ $\left(125.77 \mathrm{MHz}_{2} \mathrm{CDCl}_{3}\right) \delta 16.2\left(\mathrm{CH}_{3}\right.$ at $\left.\mathrm{C}-2\right), 17.7\left(\mathrm{CH}_{3}\right.$ at $\left.\mathrm{C}-6\right), 25.6(\mathrm{C}-1), 26.4(\mathrm{C}-8), 27.3(\mathrm{C}-5), 30.4$ $\left(\mathrm{SC}(\mathrm{O}) \mathrm{CH}_{3}\right), 39.5$ (C-4), 118.4 (C-2), 123.8 (C-5), 131.7 (C-7), 140.1 (C-3), 196.1 (SC(O) $\left.\mathrm{CH}_{3}\right)$. HRMS (ESI) calcd. for $\mathrm{C}_{12} \mathrm{H}_{20} \mathrm{OS}[\mathrm{M}+\mathrm{Na}]^{+}$235.1133; found 235.1138.

(E)-3,7-Dimethylocta-2,6-diene-1-thiol (50). A solution of 48 (846 mg, $3.98 \mathrm{mmol}$ ) in anhydrous tetrahydrofuran $(10 \mathrm{~mL})$ cooled at $0{ }^{\circ} \mathrm{C}$ was treated with portionwise with lithium aluminum hydride $(181 \mathrm{mg}, 4.74 \mathrm{mmol})$. The mixture was stirred at $0{ }^{\circ} \mathrm{C}$ for $4 \mathrm{~h}$. The reaction was quenched by addition of ethyl acetate $(2.0 \mathrm{~mL})$. Then, the mixture was partitioned between methylene chloride $(50 \mathrm{~mL})$ and an aqueous saturated solution of potassium tartrate $(50 \mathrm{~mL})$. The organic phase was washed with water $(2 \times 30 \mathrm{~mL})$, dried $\left(\mathrm{MgSO}_{4}\right)$, and the solvent was evaporated. The product was purified by column chromatography (silica gel) eluting with hexane to give $411 \mathrm{mg}$ (61\% yield) of $\mathbf{5 0}$ as a colorless oil: ${ }^{1} \mathrm{H} \mathrm{NMR}\left(500.13 \mathrm{MHz} \mathrm{CDCl}_{3}\right) \delta 1.40(\mathrm{t}, J=7.1 \mathrm{~Hz}, 1 \mathrm{H}, \mathrm{SH}), 1.60(\mathrm{~s}, 3 \mathrm{H}, \mathrm{H}-8), 1.66\left(\mathrm{~d}, J=1.2 \mathrm{~Hz}, \mathrm{CH}_{3}\right.$ at C-7), 1.68 (d, J = 1.2 Hz, $\mathrm{CH}_{3}$ at C-3), 2.00 (m, 2H, H-4), 2.07 (m, 2H, H-4), 3.16 (ddd, J = 7.6, 7.3, 0.4 Hz, 
2H, H-1), 5.08 (tq, $J=6.9,1.4 \mathrm{~Hz}, 1 \mathrm{H}, \mathrm{H}-6), 5.34$ (tsxt, $J=7.8,1.3 \mathrm{~Hz}, 1 \mathrm{H}, \mathrm{H}-2) ;{ }^{13} \mathrm{C}$ NMR $(125.77 \mathrm{MHz}$, $\left.\mathrm{CDCl}_{3}\right) \delta 15.8\left(\mathrm{CH}_{3}\right.$ at $\left.\mathrm{C}-2\right), 17.7\left(\mathrm{CH}_{3}\right.$ at C-6), $22.1(\mathrm{C}-1), 25.7(\mathrm{C}-8), 26.4(\mathrm{C}-5), 39.4(\mathrm{C}-4), 123.3(\mathrm{C}-2)$, 123.6 (C-5), 131.7 (C-7), 137.5 (C-3). HRMS (ESI) calcd. for $\mathrm{C}_{10} \mathrm{H}_{19} \mathrm{~S}[\mathrm{M}+\mathrm{H}]^{+}$171.1207; found 171.1194.

Tetraethyl 1-[((E)-3,7-dimethylocta-2,6-diene-1-thio)ethyl] 1,1-bisphosphonate (52). A solution of 21 $(1.00 \mathrm{mg}, 3.34 \mathrm{mmol})$ in anhydrous methylene chloride $(15 \mathrm{~mL})$ was treated with triethylamine $(337 \mathrm{mg}$, $470 \mu \mathrm{L}, 3.34 \mathrm{mmol})$ and $\mathbf{5 0}(580.9 \mathrm{mg}, 3.34 \mathrm{mmol})$ as described for the preparation of $\mathbf{2 2}$. The solvent was evaporated and the residue was purified by column chromatography (silica gel) employing EtOAc as eluent to give $1.163 \mathrm{~g}$ (74\% yield) of 52 as a colorless oil: ${ }^{1} \mathrm{H} \mathrm{NMR}\left(500.13 \mathrm{MHz}, \mathrm{CDCl}_{3}\right) \delta 1.35$ $\left(\mathrm{t}, J=7.1 \mathrm{~Hz}, 12 \mathrm{H}, \mathrm{CH}_{2} \mathrm{CH}_{3}\right), 1.60(\mathrm{~s}, 3 \mathrm{H}, \mathrm{H}-11), 1.67\left(\mathrm{~s}, 3 \mathrm{H}, \mathrm{CH}_{3}\right.$ at $\left.\mathrm{C}-10\right), 1.68\left(\mathrm{~s}, 3 \mathrm{H}, \mathrm{CH}_{3}\right.$ at C-6), 2.02 (m, 2H, H-7), 2.07 (m, 2H, H-8), $2.61(\mathrm{tt}, J=23.8,6.1 \mathrm{~Hz}, 1 \mathrm{H}, \mathrm{H}-1), 3.02(\mathrm{dt}, J=16.2,6.1 \mathrm{~Hz}, 2 \mathrm{H}, \mathrm{H}-2)$, $3.22(\mathrm{~d}, J=7.7 \mathrm{~Hz}, 2 \mathrm{H}, \mathrm{H}-4), 5.08(\mathrm{~m}, 1 \mathrm{H}, \mathrm{H}-9), 5.26(\mathrm{t}, J=7.7 \mathrm{~Hz}, 1 \mathrm{H}, \mathrm{H}-5) ;{ }^{13} \mathrm{C}$ NMR $(125.77 \mathrm{MHz}$, $\left.\mathrm{CDCl}_{3}\right) \delta 16.2\left(\mathrm{CH}_{3}\right.$ at C-6), $16.4\left(\mathrm{~d}, J=6.1 \mathrm{~Hz}, \mathrm{CH}_{2} \mathrm{CH}_{3}\right), 17.7\left(\mathrm{CH}_{3}\right.$ at C-10), $25.7(\mathrm{C}-11), 26.5(\mathrm{C}-8)$, $27.3(\mathrm{t}, J=5.0 \mathrm{~Hz}, \mathrm{C}-2), 30.7(\mathrm{C}-4), 38.9(\mathrm{t}, J=131.6 \mathrm{~Hz}, \mathrm{C}-1), 39.6$ (C-4), 119.9 (C-5), 123.9 (C-9), 131.7 (C-10), 139.4 (C-6); ${ }^{31} \mathrm{P}$ NMR (202.46 MHz, $\left.\mathrm{CDCl}_{3}\right) \delta 21.72 \mathrm{ppm}$. HRMS (ESI) calcd. for $\mathrm{C}_{20} \mathrm{H}_{40} \mathrm{O}_{6} \mathrm{SP}_{2}$ $[\mathrm{M}+\mathrm{Na}]^{+}$493.1919; found 493.1929.

1-[((E)-3,7-Dimethylocta-2,6-diene-1-thio)ethyl] 1,1-bisphosphonic Acid (44). A solution of the Michael adduct 52 (997 mg, $2.1 \mathrm{mmol})$ and 2,4,6-collidine $(2.53 \mathrm{~g}, 2.76 \mathrm{~mL}, 21.0 \mathrm{mmol})$ in anhydrous methylene chloride $(20 \mathrm{~mL})$ was treated with bromotrimethylsilane $(3.23 \mathrm{~g}, 2.73 \mathrm{~mL}, 21.1 \mathrm{mmol})$ under an argon atmosphere. The reaction mixture was stirred at room temperature for $48 \mathrm{~h}$. Then, methanol $(1.0 \mathrm{~mL})$ was added and the solvent was evaporated. The residue was dissolved in methanol $(10 \mathrm{~mL})$ and the mixture was stirred at room temperature for $24 \mathrm{~h}$. The solvent was evaporated and the residue redissolved/evaporated in methanol four times. The product was purified by column chromatography on reverse phase eluting with a mixture of water-acetonitrile (3:2) to give $361.2 \mathrm{mg}$ ( $48 \%$ yield) of $\mathbf{4 4}$ as an amorphous solid: ${ }^{1} \mathrm{H}$ NMR $\left(500.13 \mathrm{MHz}, \mathrm{D}_{2} \mathrm{O}\right) \delta 1.54(\mathrm{~s}, 3 \mathrm{H}, \mathrm{H}-11), 1.61\left(\mathrm{~s}, 3 \mathrm{H}, \mathrm{CH}_{3}\right.$ at C-10), 1.62 (s, 3H, $\mathrm{CH}_{3}$ at C-6), 1.94-2.08 (m, 5H, H-1, H-7, H-8), 2.93 (ddd, J = 15.1, 14.0, 7.3 Hz, 2H, H-2), 3.20 (d, $J=7.7 \mathrm{~Hz}, 2 \mathrm{H}, \mathrm{H}-4), 5.11(\mathrm{t}, J=7.0 \mathrm{~Hz}, 1 \mathrm{H}, \mathrm{H}-9), 5.30(\mathrm{t}, J=7.5 \mathrm{~Hz}, 1 \mathrm{H}, \mathrm{H}-5) ;{ }^{13} \mathrm{C}$ NMR $(125.77 \mathrm{MHz}$, $\left.\mathrm{D}_{2} \mathrm{O}\right) \delta 15.3\left(\mathrm{CH}_{3}\right.$ at C-6), $16.9\left(\mathrm{CH}_{3}\right.$ at C-10), $24.8(\mathrm{C}-11), 25.7(\mathrm{C}-8), 28.7(\mathrm{t}, J=3.7 \mathrm{~Hz}, \mathrm{C}-2), 29.9(\mathrm{C}-4)$, $40.1(\mathrm{t}, J=109.3 \mathrm{~Hz}, \mathrm{C}-1), 38.7(\mathrm{C}-4), 119.8$ (C-5), 124.2 (C-9), 133.6 (C-10), 140.2 (C-6); ${ }^{31} \mathrm{P}$ NMR (202.46 MHz, $\left.\mathrm{D}_{2} \mathrm{O}\right) \delta 15.85$ ppm. HRMS (ESI) calcd. for $\mathrm{C}_{12} \mathrm{H}_{22} \mathrm{O}_{6} \mathrm{SP}_{2} \mathrm{Na}_{3}[\mathrm{M}+\mathrm{H}]^{+}$425.0305; found 425.0298 .

S-((2E,6E)-3,7,11-Trimethyldodeca-2,6,10-trien-1-yl) ethanethioate (49). To a mixture of farnesol 47 $(1.00 \mathrm{~g}, 4.5 \mathrm{mmol})$, triphenylphosphine $(1.77 \mathrm{~g}, 6.7 \mathrm{mmol})$, thioacetic acid $(0.38 \mathrm{~mL}, 410.1 \mathrm{mg}$, $5.4 \mathrm{mmol})$ in anhydrous tetrahydrofuran $(20 \mathrm{~mL})$ cooled at $0{ }^{\circ} \mathrm{C}$ was added a solution of diisopropyl azodicarboxylate $(1.13 \mathrm{~mL}, 1.15 \mathrm{~g}, 6.7 \mathrm{mmol})$ as depicted for the preparation of 31 . The product was purified by column chromatography (silica gel) eluting with hexane-EtOAc (9:1) to give $840.0 \mathrm{mg}$ (84\% yield) of 49 as a colorless oil: $R_{\mathrm{f}} 0.38$ (hexane-EtOAc, 4:1); ${ }^{1} \mathrm{H}$ NMR $\left(500.13 \mathrm{MHz}, \mathrm{CDCl}_{3}\right) \delta 1.59$ $(\mathrm{s}, 3 \mathrm{H}, \mathrm{C}-12), 1.60\left(\mathrm{~s}, 3 \mathrm{H}, \mathrm{CH}_{3}\right.$ at C-7), $1.68\left(\mathrm{~s}, 6 \mathrm{H}, \mathrm{CH}_{3}\right.$ at $\mathrm{C}-3 \mathrm{CH}_{3}$ at $\left.\mathrm{C}-11\right), 1.95-2.10(\mathrm{~m}, 8 \mathrm{H}, \mathrm{H}-4, \mathrm{H}-5$, $\mathrm{H}-8, \mathrm{H}-9), 2.32$ (s, 3H, SC(O)CH $\mathrm{CH}_{3}, 3.54$ (t, J = 7.9 Hz, 2H, H-1), 5.08 (m, 2H, H-6, H-10), 5.21 (tq, J = 7.9, $1.1 \mathrm{~Hz}, 1 \mathrm{H}, \mathrm{H}-2) ;{ }^{13} \mathrm{C}$ NMR $(125.77 \mathrm{MHz}, \mathrm{CDCl} 3) \delta 16.0\left(\mathrm{CH}_{3}\right.$ at $\left.\mathrm{C}-3\right), 16.2\left(\mathrm{CH}_{3}\right.$ at $\left.\mathrm{C}-7\right), 17.7\left(\mathrm{CH}_{3}\right.$ at C-11), 25.9 (C-12), 26.3 (C-9), 26.7 (C-5), 27.3 (C-1), $30.4\left(\mathrm{SC}(\mathrm{O}) \mathrm{CH}_{3}\right), 39.5(\mathrm{C}-4), 39.7(\mathrm{C}-8), 118.4$ (C-2), 123.7 (C-10), 124.3 (C-6), 131.3 (C-11), 135.3 (C-7), 140.2 (C-3), 196.1 (SC(O) $\left.\mathrm{CH}_{3}\right)$. HRMS (ESI) calcd. for $\mathrm{C}_{17} \mathrm{H}_{28} \mathrm{OS}[\mathrm{M}+\mathrm{Na}]^{+}$303.1759; found 303.1757.

(2E,6E)-3,7,11-Trimethyldodeca-2,6,10-triene-1-thiol (51). A solution of 49 (840.3 $\mathrm{mg}, 3.0 \mathrm{mmol})$ in anhydrous tetrahydrofuran $(10 \mathrm{~mL})$ cooled at $0{ }^{\circ} \mathrm{C}$ was treated with portionwise with lithium aluminum hydride $(170 \mathrm{mg}, 4.5 \mathrm{mmol}$ ) as described for the preparation of $\mathbf{5 0}$ to obtain $421.8 \mathrm{mg}$ of 51 (59\% yield) as a colorless oil: ${ }^{1} \mathrm{H}$ NMR $\left(500.13 \mathrm{MHz}, \mathrm{CDCl}_{3}\right) \delta 1.42(\mathrm{t}, J=7.1 \mathrm{~Hz}, 1 \mathrm{H}, \mathrm{SH}), 1.62$ $\left(\mathrm{s}, 6 \mathrm{H}, \mathrm{C}-12, \mathrm{CH}_{3}\right.$ at $\left.\mathrm{C}-3\right), 1.68\left(\mathrm{~s}, 3 \mathrm{H}, \mathrm{CH}_{3}\right.$ at C-7), $1.71\left(\mathrm{~s}, 3 \mathrm{H}, \mathrm{CH}_{3}\right.$ at C-11), 1.99-2.14 (m, 8H, H-4, H-5, $\mathrm{H}-8, \mathrm{H}-9), 3.18(\mathrm{t}, J=7.2 \mathrm{~Hz}, 2 \mathrm{H}, \mathrm{H}-1), 5.12(\mathrm{~m}, 2 \mathrm{H}, \mathrm{H}-6, \mathrm{H}-10), 5.37(\mathrm{tq}, J=7.8,1.2 \mathrm{~Hz}, 1 \mathrm{H}, \mathrm{H}-2) ;{ }^{13} \mathrm{C}$ NMR $(125.77 \mathrm{MHz}, \mathrm{CDCl} 3) \delta 15.8\left(\mathrm{CH}_{3}\right.$ at $\left.\mathrm{C}-3\right), 16.0\left(\mathrm{CH}_{3}\right.$ at $\left.\mathrm{C}-7\right), 17.7\left(\mathrm{CH}_{3}\right.$ at $\left.\mathrm{C}-11\right), 22.1(\mathrm{C}-1), 25.7$ 
(C-12), 26.3 (C-9), 26.7 (C-5), 39.4 (C-4), 39.7 (C-8), 123.3 (C-2), 123.7 (C-10), 124.3 (C-6), 131.3 (C-11), 135.3 (C-7), 137.5 (C-3). HRMS (ESI) calcd. for $\mathrm{C}_{15} \mathrm{H}_{27} \mathrm{~S}\left[\mathrm{M}+\mathrm{H}^{+}\right.$239.1833; found 239.1820.

Tetraethyl 1-[((2E,6E)-3,7,11-trimethyldodeca-2,6,10-triene-1-thio)ethyl]-1,1-bisphosphonate (53). A solution of 21 (303 mg, $1.0 \mathrm{mmol})$ in anhydrous methylene chloride $(10 \mathrm{~mL})$ was treated with triethylamine $(112 \mathrm{mg}, 154 \mu \mathrm{L}, 1.1 \mathrm{mmol})$ and $51(241 \mathrm{mg}, 1.0 \mathrm{mmol})$ as described for the preparation of 22. The solvent was evaporated and the residue was purified by column chromatography (silica gel) employing EtOAc as eluent to yield $412.3 \mathrm{mg}$ (76\% yield) of 53 as a colorless oil: $R_{\mathrm{f}}$ 0.50 (EtOAc-methanol, 9:1); ${ }^{1} \mathrm{H}$ NMR $\left(500.13 \mathrm{MHz}, \mathrm{CDCl}_{3}\right) \delta 1.35\left(\mathrm{t}, J=7.1 \mathrm{~Hz}, 12 \mathrm{H}, \mathrm{CH}_{2} \mathrm{CH}_{3}\right), 1.36$ $\left(\mathrm{t}, J=7.0 \mathrm{~Hz}, 12 \mathrm{H}, \mathrm{CH}_{2} \mathrm{CH}_{3}^{\prime}\right), 1.60\left(\mathrm{~s}, 6 \mathrm{H}, \mathrm{C}-15, \mathrm{CH}_{3}\right.$ at C-6), $1.68\left(\mathrm{~s}, 6 \mathrm{H}, \mathrm{CH}_{3}\right.$ at $\mathrm{C}-10, \mathrm{CH}_{3}$ at C-14), 1.96-2.10 (m, 8H, H-7, H-8, H-11, H-12), $2.61(\mathrm{tt}, J=23.8,6.1 \mathrm{~Hz}, 1 \mathrm{H}, \mathrm{H}-1), 3.02(\mathrm{dt}, J=18.2,6.1 \mathrm{~Hz}, 2 \mathrm{H}$, H-2), $3.22(\mathrm{~d}, J=7.7 \mathrm{~Hz}, 2 \mathrm{H}, \mathrm{H}-4), 4.20\left(\mathrm{~m}, 8 \mathrm{H}, \mathrm{CH}_{2} \mathrm{CH}_{3}\right), 5.10(\mathrm{~m}, 2 \mathrm{H}, \mathrm{H}-9, \mathrm{H}-13), 5.26(\mathrm{tq}, J=7.6,1.0$ $\mathrm{Hz}, 1 \mathrm{H}, \mathrm{H}-5) ;{ }^{13} \mathrm{C}$ NMR $(125.77 \mathrm{MHz}, \mathrm{CDCl} 3) \delta 16.2\left(\mathrm{CH}_{3}\right.$ at C-6), $16.3\left(\mathrm{CH}_{3}\right.$ at $\left.\mathrm{C}-10\right), 16.4(\mathrm{~d}, J=6.1 \mathrm{~Hz}$, $\left.\mathrm{CH}_{2} \mathrm{CH}_{3}\right), 17.7\left(\mathrm{CH}_{3}\right.$ at C-14), $25.7(\mathrm{C}-15), 27.3(\mathrm{t}, J=5.0 \mathrm{~Hz}, \mathrm{C}-2), 26.5(\mathrm{C}-12), 26.7(\mathrm{C}-8), 30.7(\mathrm{C}-4), 38.9$ $(\mathrm{t}, J=131.6 \mathrm{~Hz}, \mathrm{C}-1), 39.6(\mathrm{C}-7), 39.7(\mathrm{C}-11), 62.7\left(\mathrm{~d}, J=6.7 \mathrm{~Hz}, \mathrm{CH}_{2} \mathrm{CH}_{3}\right), 62.8\left(\mathrm{~d}, J=6.6 \mathrm{~Hz}, \mathrm{C}^{\prime} \mathrm{H}_{2} \mathrm{CH}_{3}\right)$, 119.8 (C-5), 123.8 (C-13), 124.3 (C-9), 131.3 (C-14), 135.3 (C-10), 139.5 (C-6); ${ }^{31}$ P NMR (202.46 MHz, $\left.\mathrm{CDCl}_{3}\right) \delta$ 21.47. HRMS (ESI) calcd. for $\mathrm{C}_{25} \mathrm{H}_{48} \mathrm{O}_{6} \mathrm{SP}_{2}[\mathrm{M}+\mathrm{Na}]^{+} 561.2545$; found 561.2534.

1-[((2E,6E)-3,7,11-Trimethyldodeca-2,6,10-triene-1-thio)ethyl]-1,1-bisphosphonic Acid (45). A solution of 53 (375 mg, $0.7 \mathrm{mmol}), 2,4,6$-collidine $(843 \mathrm{mg}, 920 \mu \mathrm{L}, 7.0 \mathrm{mmol}$ ) in anhydrous methylene chloride $(10 \mathrm{~mL})$ was treated with bromotrimethylsilane $(1.07 \mathrm{~g}, 0.9 \mathrm{~mL}, 7.0 \mathrm{mmol})$ under an argon atmosphere. The reaction mixture was stirred at room temperature for $48 \mathrm{~h}$. Then, methanol $(1.0 \mathrm{~mL})$ was added and the reaction mixture was treated as depicted for the preparation of 44 . The residue was purified by reversed-phase column chromatography eluting with a mixture of water-acetonitrile (2:1) to give $170 \mathrm{mg}$ (52\% yield) of 45 as an amorphous solid: ${ }^{1} \mathrm{H}$ NMR $\left(500.13 \mathrm{MHz}, \mathrm{D}_{2} \mathrm{O}-\mathrm{CD}_{3} \mathrm{OD}\right) \delta 1.61(\mathrm{~s}, 6 \mathrm{H}$, $\mathrm{C}-15, \mathrm{CH}_{3}$ at C-6), $1.69\left(\mathrm{~s}, 3 \mathrm{H}, \mathrm{CH}_{3}\right.$ at C-10) $1.72\left(\mathrm{~s}, 3 \mathrm{H}, \mathrm{CH}_{3}\right.$ at $\left.\mathrm{C}-14\right), 1.96-2.17(\mathrm{~m}, 9 \mathrm{H}, \mathrm{H}-1, \mathrm{H}-7, \mathrm{H}-8$, $\mathrm{H}-11, \mathrm{H}-12), 3.06$ (dt, $J=14.6,7.1 \mathrm{~Hz}, 2 \mathrm{H}, \mathrm{H}-2), 3.31$ (d, $J=7.9 \mathrm{~Hz}, 2 \mathrm{H}, \mathrm{H}-4), 5.14$ (m, 2H, H-9, H-13), $5.26(\mathrm{tq}, J=7.8,1.0 \mathrm{~Hz}, 1 \mathrm{H}, \mathrm{H}-5) ;{ }^{13} \mathrm{C}$ NMR $\left(125.77 \mathrm{MHz}, \mathrm{D}_{2} \mathrm{O}_{-} \mathrm{CD}_{3} \mathrm{OD}\right) \delta 16.1\left(\mathrm{CH}_{3}\right.$ at C-6), $16.3\left(\mathrm{CH}_{3}\right.$ at C-10), $17.9\left(\mathrm{CH}_{3}\right.$ at C-14), $24.3(\mathrm{C}-15), 25.9(\mathrm{C}-12), 30.2(\mathrm{t}, J=3.7 \mathrm{~Hz}, \mathrm{C}-2), 26.9(\mathrm{C}-12), 27.0(\mathrm{C}-8), 31.4$ (C-4), 41.4 (t, J = 109.4 Hz, C-1), 40.09 (C-7), 40.11 (C-11), 120.9 (C-5), 125.23 (C-13), 125.22 (C-9), 133.4 (C-14), 136.7 (C-10), 140.6 (C-6); ${ }^{31} \mathrm{P}$ NMR (202.46 MHz, $\left.\mathrm{D}_{2} \mathrm{O}-\mathrm{CD}_{3} \mathrm{OD}\right) \delta 18.16$ ppm. HRMS (ESI) calcd. for $\mathrm{C}_{17} \mathrm{H}_{29} \mathrm{O}_{6} \mathrm{SP}_{2} \mathrm{Na}_{4}[\mathrm{M}+\mathrm{H}]^{+}$515.0751; found 515.0737.

Acetylation Reaction. General Procedure. Tetraethyl 2-( $N$-n-alkylamino)ethyl bisphosphonates 12-14, 61-65 (15 mmol) were treated individually with acetic anhydride $(3.0 \mathrm{~mL})$ and pyridine $(3.0 \mathrm{~mL})$. The mixture was stirred at room temperature overnight. The solvent was evaporated and each residue was purified by column chromatography (silica gel) eluting with $\mathrm{CH}_{2} \mathrm{Cl}_{2}$-methanol (99:1) to produce the acetylated products $67-74$.

Tetraethyl 2-(N-n-propylacetamido)ethyl-1,1-bisphosphonate (67): $66 \%$ yield; colorless oil; ${ }^{1} \mathrm{H}$ NMR $\left(500.13 \mathrm{MHz}, \mathrm{CDCl}_{3}\right) \delta 0.90(\mathrm{t}, J=7.4 \mathrm{~Hz}, 3 \mathrm{H}, \mathrm{H}-6), 1.33\left(\mathrm{t}, J=7.0 \mathrm{~Hz}, 6 \mathrm{H}, \mathrm{CH}_{2} \mathrm{CH}_{3}\right), 1.34$ $\left(\mathrm{t}, J=7.0 \mathrm{~Hz}, 6 \mathrm{H}, \mathrm{CH}_{2} \mathrm{CH}_{3}^{\prime}\right), 1.55(\mathrm{p}, J=7.1 \mathrm{~Hz}, 2 \mathrm{H}, \mathrm{H}-5), 2.06\left(\mathrm{~s}, 3 \mathrm{H}, \mathrm{C}(\mathrm{O}) \mathrm{CH}_{3}\right), 3.38(\mathrm{t}, J=7.7 \mathrm{~Hz}$, $1 \mathrm{H}, \mathrm{H}-4), 3.46(\mathrm{tt}, J=22.6,7.5 \mathrm{~Hz}, 1 \mathrm{H}, \mathrm{H}-1), 3.74(\mathrm{ddd}, J=14.7,11.2,7.4 \mathrm{~Hz}, 2 \mathrm{H}, \mathrm{H}-2), 4.17(\mathrm{~m}$, $\left.8 \mathrm{H}, \mathrm{CH}_{2} \mathrm{CH}_{3}\right) ;{ }^{13} \mathrm{C}$ NMR $\left(125.77 \mathrm{MHz}, \mathrm{CDCl}_{3}\right) \delta 11.2(\mathrm{C}-6), 16.30\left(\mathrm{~d}, J=4.1 \mathrm{~Hz}, \mathrm{CH}_{2} \mathrm{CH}_{3}\right), 16.36(\mathrm{~d}$, $\left.J=4.1 \mathrm{~Hz}, \mathrm{CH}_{2} \mathrm{CH}_{3}\right), 21.6\left(\left(\mathrm{C}(\mathrm{O}) \mathrm{CH}_{3}\right), 22.2(\mathrm{C}-5), 34.1(\mathrm{t}, J=130.1 \mathrm{~Hz}, \mathrm{C}-1), 44.6(\mathrm{t}, J=3.3 \mathrm{~Hz}, \mathrm{C}-2)\right.$, $52.7(\mathrm{C}-4), 62.4\left(\mathrm{~d}, \mathrm{~J}=6.8 \mathrm{~Hz}, \mathrm{CH}_{2} \mathrm{CH}_{3}\right), 63.0\left(\mathrm{~d}, \mathrm{~J}=6.7 \mathrm{~Hz}, \mathrm{CH}_{2} \mathrm{CH}_{3}\right), 171.1\left(\mathrm{C}(\mathrm{O}) \mathrm{CH}_{3}\right) ;{ }^{31} \mathrm{P}$ NMR $\left(202.46 \mathrm{MHz}, \mathrm{CDCl}_{3}\right) \delta 21.48$.

Tetraethyl 2-(N-n-butylacetamido)ethyl-1,1-bisphosphonate (68): 51\% yield; colorless oil; ${ }^{1} \mathrm{H}$ NMR $\left(500.13 \mathrm{MHz}, \mathrm{CDCl}_{3}\right) \delta 0.95(\mathrm{t}, J=7.3 \mathrm{~Hz}, 3 \mathrm{H}, \mathrm{H}-7), 1.34\left(\mathrm{t}, J=7.0 \mathrm{~Hz}, 6 \mathrm{H}, \mathrm{CH}_{2} \mathrm{CH}_{3}\right), 1.35$ $\left(\mathrm{t}, J=7.1 \mathrm{~Hz}, 6 \mathrm{H}, \mathrm{CH}_{2} \mathrm{CH}_{3}^{\prime}\right), 1.53(\mathrm{p}, J=7.1 \mathrm{~Hz}, 2 \mathrm{H}, \mathrm{H}-5), 2.07\left(\mathrm{~s}, 3 \mathrm{H}, \mathrm{C}(\mathrm{O}) \mathrm{CH}_{3}\right), 3.41(\mathrm{t}, J=7.8 \mathrm{~Hz}$, $1 \mathrm{H}, \mathrm{H}-4), 3.44(\mathrm{tt}, J=22.8,7.4 \mathrm{~Hz}, 1 \mathrm{H}, \mathrm{H}-1), 3.74(\mathrm{ddd}, J=14.7,11.1,7.4 \mathrm{~Hz}, 2 \mathrm{H}, \mathrm{H}-2), 4.19(\mathrm{~m}$, $\left.8 \mathrm{H}, \mathrm{CH}_{2} \mathrm{CH}_{3}\right) ;{ }^{13} \mathrm{C} \mathrm{NMR}\left(125.77 \mathrm{MHz}, \mathrm{CDCl}_{3}\right) \delta 13.8(\mathrm{C}-7), 16.27\left(\mathrm{~d}, J=4.1 \mathrm{~Hz}, \mathrm{CH}_{2} \mathrm{CH}_{3}\right), 16.33(\mathrm{~d}$, $\left.J=4.1 \mathrm{~Hz}, \mathrm{CH}_{2} \mathrm{CH}_{3}\right), 20.0(\mathrm{C}-6), 21.6\left(\left(\mathrm{C}(\mathrm{O}) \mathrm{CH}_{3}\right), 31.1(\mathrm{C}-5), 34.1(\mathrm{t}, J=130.1 \mathrm{~Hz}, \mathrm{C}-1), 44.6(\mathrm{t}, J=3.3 \mathrm{~Hz}\right.$, 
$\mathrm{C}-2), 50.6(\mathrm{C}-4), 62.4\left(\mathrm{~d}, J=6.8 \mathrm{~Hz}, \mathrm{CH}_{2} \mathrm{CH}_{3}\right), 62.9\left(\mathrm{~d}, J=6.8 \mathrm{~Hz}, \mathrm{CH}_{2} \mathrm{CH}_{3}\right), 171.0\left(\mathrm{C}(\mathrm{O}) \mathrm{CH}_{3}\right) ;{ }^{31} \mathrm{P} \mathrm{NMR}$ (202.46 MHz, $\left.\mathrm{CDCl}_{3}\right) \delta 21.47$.

Tetraethyl 2-(N-n-penylacetamido)ethyl-1,1-bisphosphonate (69): $47 \%$ yield, colorless oil; ${ }^{1} \mathrm{H}$ NMR $\left(500.13 \mathrm{MHz}, \mathrm{CDCl}_{3}\right) \delta 0.91(\mathrm{t}, J=7.2 \mathrm{~Hz}, 3 \mathrm{H}, \mathrm{H}-8), 1.26\left(\mathrm{~m}, 2 \mathrm{H}, \mathrm{CH}_{2}\right), 1.34(\mathrm{t}, J=7.1 \mathrm{~Hz}, 6 \mathrm{H}$, $\left.\mathrm{CH}_{2} \mathrm{CH}_{3}\right), 1.35\left(\mathrm{t}, J=7.1 \mathrm{~Hz}, 6 \mathrm{H}, \mathrm{CH}_{2} \mathrm{CH}_{3}^{\prime}\right), 1.53(\mathrm{p}, J=7.7 \mathrm{~Hz}, 2 \mathrm{H}, \mathrm{H}-5), 2.07\left(\mathrm{~s}, 3 \mathrm{H}, \mathrm{C}(\mathrm{O}) \mathrm{CH}_{3}\right), 3.41$ $(\mathrm{t}, J=7.7 \mathrm{~Hz}, 1 \mathrm{H}, \mathrm{H}-4), 3.44(\mathrm{tt}, J=22.8,7.4 \mathrm{~Hz}, 1 \mathrm{H}, \mathrm{H}-1), 3.74(\mathrm{ddd}, J=14.7,11.1,7.4 \mathrm{~Hz}, 2 \mathrm{H}, \mathrm{H}-2)$, $4.19\left(\mathrm{~m}, 8 \mathrm{H}, \mathrm{CH}_{2} \mathrm{CH}_{3}\right) ;{ }^{13} \mathrm{C} \mathrm{NMR}\left(125.77 \mathrm{MHz}, \mathrm{CDCl}_{3}\right) \delta 13.9(\mathrm{C}-8), 16.3\left(\mathrm{~d}, J=4.2 \mathrm{~Hz}, \mathrm{CH}_{2} \mathrm{CH}_{3}\right), 16.4$ $\left(\mathrm{d}, J=4.2 \mathrm{~Hz}, \mathrm{CH}_{2} \mathrm{CH}_{3}\right), 21.6\left(\left(\mathrm{C}(\mathrm{O}) \mathrm{CH}_{3}\right), 22.4(\mathrm{C}-7), 28.7(\mathrm{C}-6), 28.9(\mathrm{C}-5), 34.1(\mathrm{t}, J=130.0 \mathrm{~Hz}, \mathrm{C}-1)\right.$, $44.6(\mathrm{t}, J=3.4 \mathrm{~Hz}, \mathrm{C}-2), 50.9(\mathrm{C}-4), 62.4\left(\mathrm{~d}, J=6.8 \mathrm{~Hz}, \mathrm{CH}_{2} \mathrm{CH}_{3}\right), 62.9\left(\mathrm{~d}, J=6.7 \mathrm{~Hz}, \mathrm{CH}_{2} \mathrm{CH}_{3}\right), 171.0$ $\left(\mathrm{C}(\mathrm{O}) \mathrm{CH}_{3}\right) ;{ }^{31} \mathrm{P} \mathrm{NMR}\left(202.46 \mathrm{MHz}, \mathrm{CDCl}_{3}\right) \delta 21.47$.

Tetraethyl 2-(N-n-hexylacetamido)ethyl-1,1-bisphosphonate (70): 51\% yield, colorless oil; ${ }^{1} \mathrm{H}$ NMR $\left(500.13 \mathrm{MHz}, \mathrm{CDCl}_{3}\right) \delta 0.87(\mathrm{t}, J=6.9 \mathrm{~Hz}, 3 \mathrm{H}, \mathrm{H}-9), 1.29\left(\mathrm{~m}, 6 \mathrm{H}, \mathrm{CH}_{2}\right), 1.34(\mathrm{t}, J=7.0 \mathrm{~Hz}, 6 \mathrm{H}$, $\left.\mathrm{CH}_{2} \mathrm{CH}_{3}\right), 1.35\left(\mathrm{t}, J=7.1 \mathrm{~Hz}, 6 \mathrm{H}, \mathrm{CH}_{2} \mathrm{CH}_{3}^{\prime}\right), 1.52(\mathrm{~m}, 2 \mathrm{H}, \mathrm{H}-5), 2.07\left(\mathrm{~s}, 3 \mathrm{H}, \mathrm{C}(\mathrm{O}) \mathrm{CH}_{3}\right), 3.41(\mathrm{t}, J=7.7 \mathrm{~Hz}$, $1 \mathrm{H}, \mathrm{H}-4), 3.44(\mathrm{tt}, J=22.8,7.4 \mathrm{~Hz}, 1 \mathrm{H}, \mathrm{H}-1), 3.74(\mathrm{ddd}, J=14.7,11.1,7.4 \mathrm{~Hz}, 2 \mathrm{H}, \mathrm{H}-2), 4.18(\mathrm{~m}, 8 \mathrm{H}$, $\left.\mathrm{CH}_{2} \mathrm{CH}_{3}\right) ;{ }^{13} \mathrm{C} \mathrm{NMR}\left(125.77 \mathrm{MHz}, \mathrm{CDCl}_{3}\right) \delta 14.0(\mathrm{C}-9), 16.2\left(\mathrm{~d}, J=4.3 \mathrm{~Hz}, \mathrm{CH}_{2} \mathrm{CH}_{3}\right), 16.4(\mathrm{~d}, J=4.1 \mathrm{~Hz}$, $\left.\mathrm{CH}_{2} \mathrm{CH}_{3}\right), 21.6\left(\left(\mathrm{C}(\mathrm{O}) \mathrm{CH}_{3}\right), 22.6(\mathrm{C}-8), 26.5(\mathrm{C}-6), 29.0(\mathrm{C}-5), 31.5(\mathrm{C}-7), 34.1(\mathrm{t}, J=130.0 \mathrm{~Hz}, \mathrm{C}-1), 44.6\right.$ $(\mathrm{t}, J=3.2 \mathrm{~Hz}, \mathrm{C}-2), 50.9(\mathrm{C}-4), 62.4\left(\mathrm{~d}, J=6.8 \mathrm{~Hz}, \mathrm{CH}_{2} \mathrm{CH}_{3}\right), 62.9\left(\mathrm{~d}, J=6.7 \mathrm{~Hz}, \mathrm{CH}_{2} \mathrm{CH}_{3}\right), 171.0$ $\left(\mathrm{C}(\mathrm{O}) \mathrm{CH}_{3}\right) ;{ }^{31} \mathrm{P} \mathrm{NMR}\left(202.46 \mathrm{MHz}, \mathrm{CDCl}_{3}\right) \delta 21.48$.

Tetraethyl 2-(N-n-heptylacetamido)ethyl-1,1-bisphosphonate (71): $66 \%$ yield; colorless oil; ${ }^{1} \mathrm{H}$ NMR $\left(500.13 \mathrm{MHz}, \mathrm{CDCl}_{3}\right) \delta 0.89(\mathrm{t}, J=7.1 \mathrm{~Hz}, 3 \mathrm{H}, \mathrm{H}-10), 1.29\left(\mathrm{~m}, 8 \mathrm{H}, \mathrm{CH}_{2}\right), 1.34(\mathrm{t}, J=7.1 \mathrm{~Hz}, 6 \mathrm{H}$, $\left.\mathrm{CH}_{2} \mathrm{CH}_{3}\right), 1.35\left(\mathrm{t}, J=7.1 \mathrm{~Hz}, 6 \mathrm{H}, \mathrm{CH}_{2} \mathrm{CH}_{3}^{\prime}\right), 1.52(\mathrm{p}, J=7.5 \mathrm{~Hz}, 2 \mathrm{H}, \mathrm{H}-5), 2.07\left(\mathrm{~s}, 3 \mathrm{H}, \mathrm{C}(\mathrm{O}) \mathrm{CH}_{3}\right), 3.41$ $(\mathrm{t}, J=7.7 \mathrm{~Hz}, 1 \mathrm{H}, \mathrm{H}-4), 3.44(\mathrm{tt}, J=23.1,7.4 \mathrm{~Hz}, 1 \mathrm{H}, \mathrm{H}-1), 3.74(\mathrm{ddd}, J=14.7,11.1,7.4 \mathrm{~Hz}, 2 \mathrm{H}, \mathrm{H}-2)$, $4.18\left(\mathrm{~m}, 8 \mathrm{H}, \mathrm{CH}_{2} \mathrm{CH}_{3}\right) ;{ }^{13} \mathrm{C}$ NMR $\left(125.77 \mathrm{MHz}, \mathrm{CDCl}_{3}\right) \delta 14.0(\mathrm{C}-10), 16.3\left(\mathrm{~d}, J=4.4 \mathrm{~Hz}, \mathrm{CH}_{2} \mathrm{CH}_{3}\right), 16.4$ $\left(\mathrm{d}, \mathrm{J}=4.1 \mathrm{~Hz}, \mathrm{CH}_{2} \mathrm{CH}_{3}\right), 21.6\left(\left(\mathrm{C}(\mathrm{O}) \mathrm{CH}_{3}\right), 22.6\right.$ (C-9), 26.8 (C-6), 29.03 (C-5), 29.04 (C-7), 31.5 (C-8), 34.1 $(\mathrm{t}, J=130.0 \mathrm{~Hz}, \mathrm{C}-1), 44.6(\mathrm{C}-2), 50.9(\mathrm{C}-4), 62.4\left(\mathrm{~d}, J=6.6 \mathrm{~Hz}, \mathrm{CH}_{2} \mathrm{CH}_{3}\right), 62.9\left(\mathrm{~d}, J=6.8 \mathrm{~Hz}, \mathrm{CH}_{2} \mathrm{CH}_{3}\right)$, $171.0\left(\mathrm{C}(\mathrm{O}) \mathrm{CH}_{3}\right) ;{ }^{31} \mathrm{P}$ NMR $\left(202.46 \mathrm{MHz}, \mathrm{CDCl}_{3}\right) \delta 21.49$.

Tetraethyl 2-(N-n-octylacetamido)ethyl-1,1-bisphosphonate (72): 50\% yield; colorless oil; ${ }^{1} \mathrm{H}$ NMR $\left(500.13 \mathrm{MHz}, \mathrm{CDCl}_{3}\right) \delta 0.89(\mathrm{t}, J=7.0 \mathrm{~Hz}, 3 \mathrm{H}, \mathrm{H}-11), 1.29\left(\mathrm{~m}, 10 \mathrm{H}, \mathrm{CH}_{2}\right), 1.34(\mathrm{t}, J=7.1 \mathrm{~Hz}, 6 \mathrm{H}$, $\left.\mathrm{CH}_{2} \mathrm{CH}_{3}\right), 1.35\left(\mathrm{t}, J=7.1 \mathrm{~Hz}, 6 \mathrm{H}, \mathrm{CH}_{2} \mathrm{CH}_{3}^{\prime}\right), 1.52(\mathrm{p}, J=7.4 \mathrm{~Hz}, 2 \mathrm{H}, \mathrm{H}-5), 2.07\left(\mathrm{~s}, 3 \mathrm{H}, \mathrm{C}(\mathrm{O}) \mathrm{CH}_{3}\right), 3.41$ $(\mathrm{t}, J=7.8 \mathrm{~Hz}, 1 \mathrm{H}, \mathrm{H}-4), 3.44(\mathrm{tt}, J=22.9,7.4 \mathrm{~Hz}, 1 \mathrm{H}, \mathrm{H}-1), 3.74(\mathrm{ddd}, J=14.7,11.1,7.4 \mathrm{~Hz}, 2 \mathrm{H}, \mathrm{H}-2)$, $4.19\left(\mathrm{~m}, 8 \mathrm{H}, \mathrm{CH}_{2} \mathrm{CH}_{3}\right) ;{ }^{13} \mathrm{C}$ NMR $\left(125.77 \mathrm{MHz}, \mathrm{CDCl}_{3}\right) \delta 14.0(\mathrm{C}-11), 16.2\left(\mathrm{~d}, J=3.9 \mathrm{~Hz}, \mathrm{CH}_{2} \mathrm{CH}_{3}\right), 16.3$ $\left(\mathrm{d}, J=3.9 \mathrm{~Hz}, \mathrm{CH}_{2} \mathrm{CH}_{3}\right), 21.5\left(\left(\mathrm{C}(\mathrm{O}) \mathrm{CH}_{3}\right), 22.5(\mathrm{C}-10), 26.7(\mathrm{C}-6), 28.9(\mathrm{C}-8), 29.1\right.$ (C-5), $29.2(\mathrm{C}-7), 31.6$ (C-9), $34.0(\mathrm{t}, J=130.0 \mathrm{~Hz}, \mathrm{C}-1), 44.5(\mathrm{t}, J=3.2 \mathrm{~Hz}, \mathrm{C}-2), 50.8(\mathrm{C}-4), 62.3\left(\mathrm{~d}, J=6.8 \mathrm{~Hz}, \mathrm{CH}_{2} \mathrm{CH}_{3}\right), 62.8$ $\left(\mathrm{d}, \mathrm{J}=6.6 \mathrm{~Hz}, \mathrm{CH}_{2} \mathrm{CH}_{3}\right), 170.9\left(\mathrm{C}(\mathrm{O}) \mathrm{CH}_{3}\right) ;{ }^{31} \mathrm{P} \mathrm{NMR}\left(202.46 \mathrm{MHz}, \mathrm{CDCl}_{3}\right) \delta 21.44$.

Tetraethyl 2-(N-n-nonylacetamido)ethyl-1,1-bisphosphonate (73): 57\% yield; colorless oil; ${ }^{1} \mathrm{H}$ NMR $\left(500.13 \mathrm{MHz}, \mathrm{CDCl}_{3}\right) \delta 0.84(\mathrm{t}, J=6.9 \mathrm{~Hz}, 3 \mathrm{H}, \mathrm{H}-12), 1.23\left(\mathrm{~m}, 12 \mathrm{H}, \mathrm{CH}_{2}\right), 1.30(\mathrm{t}, J=7.1 \mathrm{~Hz}, 6 \mathrm{H}$, $\left.\mathrm{CH}_{2} \mathrm{CH}_{3}\right), 1.31\left(\mathrm{t}, J=7.1 \mathrm{~Hz}, 6 \mathrm{H}, \mathrm{CH}_{2} \mathrm{CH}_{3}^{\prime}\right), 1.49(\mathrm{p}, J=7.1 \mathrm{~Hz}, 2 \mathrm{H}, \mathrm{H}-5), 2.03\left(\mathrm{~s}, 3 \mathrm{H}, \mathrm{C}(\mathrm{O}) \mathrm{CH}_{3}\right), 3.37$ $(\mathrm{t}, J=7.8 \mathrm{~Hz}, 1 \mathrm{H}, \mathrm{H}-4), 3.40$ (tt, $J=23.0,7.4 \mathrm{~Hz}, 1 \mathrm{H}, \mathrm{H}-1), 3.70(\mathrm{ddd}, J=14.8,11.2,7.4 \mathrm{~Hz}, 2 \mathrm{H}, \mathrm{H}-2)$, $4.15\left(\mathrm{~m}, 8 \mathrm{H}, \mathrm{CH}_{2} \mathrm{CH}_{3}\right) ;{ }^{13} \mathrm{C}$ NMR $\left(125.77 \mathrm{MHz}, \mathrm{CDCl}_{3}\right) \delta 14.0(\mathrm{C}-12), 16.2\left(\mathrm{~d}, J=4.0 \mathrm{~Hz}, \mathrm{CH}_{2} \mathrm{CH}_{3}\right), 16.3$ $\left(\mathrm{d}, J=3.9 \mathrm{~Hz}, \mathrm{CH}_{2} \mathrm{CH}_{3}\right), 21.5\left(\left(\mathrm{C}(\mathrm{O}) \mathrm{CH}_{3}\right), 22.5(\mathrm{C}-11), 26.7(\mathrm{C}-6), 29.0(\mathrm{C}-8), 29.1\right.$ (C-5), $29.3(\mathrm{C}-7), 29.4$ (C-9), $31.8(\mathrm{C}-10), 34.0$ (t, $J=130.1 \mathrm{~Hz}, \mathrm{C}-1), 44.5$ (t, $J=3.3 \mathrm{~Hz}, \mathrm{C}-2), 50.9(\mathrm{C}-4), 62.3(\mathrm{~d}, J=6.9 \mathrm{~Hz}$, $\left.\mathrm{CH}_{2} \mathrm{CH}_{3}\right), 62.9\left(\mathrm{~d}, J=6.7 \mathrm{~Hz}, \mathrm{CH}_{2} \mathrm{CH}_{3}\right), 170.9\left(\mathrm{C}(\mathrm{O}) \mathrm{CH}_{3}\right) ;{ }^{31} \mathrm{P} \mathrm{NMR}\left(202.46 \mathrm{MHz}, \mathrm{CDCl}_{3}\right) \delta 21.45$.

Tetraethyl 2-(N-n-decylacetamido)ethyl-1,1-bisphosphonate (74): 73\% yield; colorless oil; ${ }^{1} \mathrm{H}$ NMR $\left(500.13 \mathrm{MHz}, \mathrm{CDCl}_{3}\right) \delta 0.85(\mathrm{t}, J=6.9 \mathrm{~Hz}, 3 \mathrm{H}, \mathrm{H}-13), 1.23\left(\mathrm{~m}, 14 \mathrm{H}, \mathrm{CH}_{2}\right), 1.31(\mathrm{t}, J=7.0 \mathrm{~Hz}, 6 \mathrm{H}$, $\left.\mathrm{CH}_{2} \mathrm{CH}_{3}\right), 1.32\left(\mathrm{t}, J=7.1 \mathrm{~Hz}, 6 \mathrm{H}, \mathrm{CH}_{2} \mathrm{CH}_{3}^{\prime}\right), 1.49(\mathrm{p}, J=7.1 \mathrm{~Hz}, 2 \mathrm{H}, \mathrm{H}-5), 2.04\left(\mathrm{~s}, 3 \mathrm{H}, \mathrm{C}(\mathrm{O}) \mathrm{CH}_{3}\right), 3.38$ $(\mathrm{t}, J=7.5 \mathrm{~Hz}, 1 \mathrm{H}, \mathrm{H}-4), 3.44(\mathrm{tt}, J=23.2,7.6 \mathrm{~Hz}, 1 \mathrm{H}, \mathrm{H}-1), 3.71(\mathrm{ddd}, J=14.6,11.2,7.5 \mathrm{~Hz}, 2 \mathrm{H}, \mathrm{H}-2), 4.15$ $\left(\mathrm{m}, 8 \mathrm{H}, \mathrm{CH}_{2} \mathrm{CH}_{3}\right) ;{ }^{13} \mathrm{C}$ NMR $\left(125.77 \mathrm{MHz}, \mathrm{CDCl}_{3}\right) \delta 14.0(\mathrm{C}-13), 16.27\left(\mathrm{~d}, \mathrm{~J}=6.8 \mathrm{~Hz}, \mathrm{CH}_{2} \mathrm{CH}_{3}\right), 16.30(\mathrm{~d}$, $\left.J=6.6 \mathrm{~Hz}, \mathrm{CH}_{2} \mathrm{CH}_{3}\right), 21.6\left(\left(\mathrm{C}(\mathrm{O}) \mathrm{CH}_{3}\right), 22.6(\mathrm{C}-12), 26.8(\mathrm{C}-6), 29.0(\mathrm{C}-8), 29.2(\mathrm{C}-5), 29.3(\mathrm{C}-7), 29.4\right.$ (C-9), 
29.5 (C-10), $31.8(\mathrm{C}-11), 34.0(\mathrm{t}, J=130.0 \mathrm{~Hz}, \mathrm{C}-1), 44.5(\mathrm{t}, J=3.3 \mathrm{~Hz}, \mathrm{C}-2), 50.9$ (C-4), $62.4(\mathrm{~d}, J=6.9 \mathrm{~Hz}$, $\left.\mathrm{CH}_{2} \mathrm{CH}_{3}\right), 62.9\left(\mathrm{~d}, \mathrm{~J}=6.7 \mathrm{~Hz}, \mathrm{CH}_{2} \mathrm{CH}_{3}\right), 171.0\left(\mathrm{C}(\mathrm{O}) \mathrm{CH}_{3}\right) ;{ }^{31} \mathrm{P} \mathrm{NMR}\left(202.46 \mathrm{MHz}, \mathrm{CDCl}_{3}\right) \delta 21.46$.

Fluorination Reaction. General Procedure. Sodium hydride (10.5 mmol, 60\% in mineral oil) was washed with anhydrous hexane under an argon atmosphere, and then anhydrous tetrahydrofuran $(10 \mathrm{~mL})$ was added. The mixture, cooled at $0{ }^{\circ} \mathrm{C}$, was treated with the corresponding tetraethyl 2-( $N$ - $n$-alkylacetamido)ethyl-1,1-bisphosphonate such as 67-74 $(10.0 \mathrm{mmol})$ in anhydrous tetrahydrofuran $(10 \mathrm{~mL})$. The solution was stirred at $0{ }^{\circ} \mathrm{C}$ for $15 \mathrm{~min}$, then, the mixture was allowed to reach room temperature and stirred for additional $60 \mathrm{~min}$. The mixture was cooled at $0{ }^{\circ} \mathrm{C}$ and Selectfluor $(12.5 \mathrm{mmol})$ was added followed by addition of $N, N$-dimethylformamide $(5.0 \mathrm{~mL})$. The reaction mixture was allowed to reach room temperature and stirred for $4 \mathrm{~h}$. The mixture was partitioned between methylene chloride $(30 \mathrm{~mL})$ and brine $(30 \mathrm{~mL})$. The aqueous phase was extracted with methylene chloride $(2 \times 30 \mathrm{~mL})$. The combined organic layers were washed with brine $(5 \times 30 \mathrm{~mL})$, water $(2 \times 25 \mathrm{~mL})$, dried $\left(\mathrm{MgSO}_{4}\right)$, and the solvent was evaporated. The residue was purified by column chromatography eluting with a mixture of EtOAc-methanol (49:1) to produce the corresponding 1-fluoro tetraethyl esters 75-82.

Tetraethyl 2-(N-n-propylacetamido)ethyl-1-fluoro-1,1-bisphosphonate (75): $50 \%$ yield; ${ }^{1} \mathrm{H}$ NMR $(500.13 \mathrm{MHz}$, $\left.\mathrm{CDCl}_{3}\right) \delta 0.88(\mathrm{t}, J=7.3 \mathrm{~Hz}, 3 \mathrm{H}, \mathrm{H}-6), 0.91\left(\mathrm{t}, J=7.4 \mathrm{~Hz}, 3 \mathrm{H}, \mathrm{H}-6^{\prime}\right), 1.38\left(\mathrm{t}, J=7.0 \mathrm{~Hz}, 12 \mathrm{H}, \mathrm{CH}_{2} \mathrm{CH}_{3}\right)$, $1.39\left(\mathrm{dt}, J=6.9,3.3 \mathrm{~Hz}, 12 \mathrm{H}, \mathrm{CH}_{2} \mathrm{CH}_{3}^{\prime}\right), 1.53$ (sext, $\left.J=7.6 \mathrm{~Hz}, 2 \mathrm{H}, \mathrm{H}-5\right), 1.60$ (sext, $J=7.5 \mathrm{~Hz}, 2 \mathrm{H}$, $\left.\mathrm{H}-5^{\prime}\right), 2.14\left(\mathrm{~s}, 3 \mathrm{H}, \mathrm{C}(\mathrm{O}) \mathrm{CH}_{3}\right), 2.21\left(\mathrm{~s}, 3 \mathrm{H}, \mathrm{C}(\mathrm{O}) \mathrm{CH}_{3}\right), 3.45(\mathrm{t}, J=7.8 \mathrm{~Hz}, 2 \mathrm{H}, \mathrm{H}-4), 3.47(\mathrm{t}, J=7.9 \mathrm{~Hz}, 2 \mathrm{H}$, $\left.\mathrm{H}-4^{\prime}\right), 4.10(\mathrm{dd}, J=10.1,8.8 \mathrm{~Hz}, 2 \mathrm{H}, \mathrm{H}-2), 4.15\left(\mathrm{dd}, J=10.2,8.8 \mathrm{~Hz}, 2 \mathrm{H}, \mathrm{H}-2^{\prime}\right), 4.27\left(\mathrm{~m}, 8 \mathrm{H}, \mathrm{CH}_{2} \mathrm{CH}_{3}\right)$, two rotamers; ${ }^{13} \mathrm{C}$ NMR $\left(125.77 \mathrm{MHz}, \mathrm{CDCl}_{3}\right) \delta 11.1(\mathrm{C}-6), 11.3\left(\mathrm{C}-6^{\prime}\right), 16.3\left(\mathrm{t}, J=3.3 \mathrm{~Hz}, \mathrm{CH}_{2} \mathrm{CH}_{3}\right)$, $16.4\left(\mathrm{t}, J=2.3 \mathrm{~Hz}, \mathrm{CH}_{2} \mathrm{CH}_{3}^{\prime}\right), 19.8(\mathrm{C}-5), 21.2\left(\mathrm{C}-5^{\prime}\right), 21.4\left(\mathrm{C}(\mathrm{O}) \mathrm{CH}_{3}\right), 21.9\left(\mathrm{~d}, J=3.5 \mathrm{~Hz}, \mathrm{C}(\mathrm{O}) \mathrm{CH}_{3}^{\prime}\right)$, $44.9(\mathrm{~d}, J=17.9 \mathrm{~Hz}, \mathrm{C}-2), 48.4(\mathrm{~d}, J=3.5 \mathrm{~Hz}, \mathrm{C}-4), 48.9\left(\mathrm{~d}, J=18.3 \mathrm{~Hz}, \mathrm{C}-2^{\prime}\right), 50.9\left(\mathrm{~d}, J=4.2 \mathrm{~Hz}, \mathrm{C}-2^{\prime}\right)$, $64.2\left(\mathrm{~m}, \mathrm{CH}_{2} \mathrm{CH}_{3}\right), 64.7\left(\mathrm{t}, J=3.4 \mathrm{~Hz}, \mathrm{CH}_{2} \mathrm{CH}_{3}\right), 171.3\left(\mathrm{C}(\mathrm{O}) \mathrm{CH}_{3}\right), 171.6\left(\mathrm{C}(\mathrm{O})^{\prime} \mathrm{CH}_{3}\right)$, two rotamers; ${ }^{31} \mathrm{P}$ NMR $\left(202.46 \mathrm{MHz}, \mathrm{CDCl}_{3}\right) \delta 11.70(\mathrm{~d}, J=70.2 \mathrm{~Hz}), 11.90(\mathrm{~d}, J=77.4 \mathrm{~Hz})$, two rotamers; ${ }^{19} \mathrm{~F}$ NMR $\left(470.59 \mathrm{MHz}, \mathrm{CDCl}_{3}\right) \delta-192.55(\mathrm{t}, J=77.4 \mathrm{~Hz}),-192.80(\mathrm{t}, J=71.3 \mathrm{~Hz})$, two rotamers.

Tetraethyl 2-(N-n-butylacetamido)ethyl-1-fluoro-1,1-bisphosphonate (76): $65 \%$ yield; colorless oil; ${ }^{1} \mathrm{H}$ NMR $\left(500.13 \mathrm{MHz}, \mathrm{CDCl}_{3}\right) \delta 0.91(\mathrm{t}, J=7.3 \mathrm{~Hz}, 3 \mathrm{H}, \mathrm{H}-7), 0.94\left(\mathrm{t}, J=7.4 \mathrm{~Hz}, 3 \mathrm{H}, \mathrm{H}-7^{\prime}\right), 1.30(\mathrm{~m}, 2 \mathrm{H}, \mathrm{H}-6), 1.37$ $\left(\mathrm{t}, J=6.8 \mathrm{~Hz}, 12 \mathrm{H}, \mathrm{CH}_{2} \mathrm{CH}_{3}\right), 1.38\left(\mathrm{dt}, J=6.8,3.3 \mathrm{~Hz}, 12 \mathrm{H}, \mathrm{CH}_{2} \mathrm{CH}_{3}^{\prime}\right), 1.49(\mathrm{p}, J=7.7 \mathrm{~Hz}, 2 \mathrm{H}, \mathrm{H}-5), 1.54$ $\left(\mathrm{p}, J=7.8 \mathrm{~Hz}, 2 \mathrm{H}, \mathrm{H}-5^{\prime}\right), 2.12\left(\mathrm{~s}, 3 \mathrm{H}, \mathrm{C}(\mathrm{O}) \mathrm{CH}_{3}\right), 2.20\left(\mathrm{~s}, 3 \mathrm{H}, \mathrm{C}(\mathrm{O}) \mathrm{CH}_{3}\right), 3.46(\mathrm{t}, J=7.9 \mathrm{~Hz}, 2 \mathrm{H}, \mathrm{H}-4), 3.49$ $\left(\mathrm{t}, J=7.9 \mathrm{~Hz}, 2 \mathrm{H}, \mathrm{H}-4^{\prime}\right), 4.10(\mathrm{dd}, J=10.5,8.5 \mathrm{~Hz}, 2 \mathrm{H}, \mathrm{H}-2), 4.14\left(\mathrm{dd}, J=10.3,8.9 \mathrm{~Hz}, 2 \mathrm{H}, \mathrm{H}-2^{\prime}\right), 4.27$ $\left(\mathrm{m}, 8 \mathrm{H}, \mathrm{CH}_{2} \mathrm{CH}_{3}\right)$, two rotamers; ${ }^{13} \mathrm{C}$ NMR $\left(125.77 \mathrm{MHz}, \mathrm{CDCl}_{3}\right) \delta 13.75(\mathrm{C}-7), 13.84\left(\mathrm{C}-7^{\prime}\right), 16.39$ $\left(\mathrm{t}, J=3.2 \mathrm{~Hz}, \mathrm{CH}_{2} \mathrm{CH}_{3}\right), 16.44\left(\mathrm{t}, J=2.6 \mathrm{~Hz}, \mathrm{CH}_{2} \mathrm{CH}_{3}^{\prime}\right), 20.0(\mathrm{C}-6), 20.1\left(\mathrm{C}-6^{\prime}\right), 21.5\left(\mathrm{C}(\mathrm{O}) \mathrm{CH}_{3}\right), 22.0$ $\left(\mathrm{d}, J=3.6 \mathrm{~Hz}, \mathrm{C}(\mathrm{O}) \mathrm{CH}_{3}^{\prime}\right), 28.7(\mathrm{C}-5), 30.1\left(\mathrm{C}-5^{\prime}\right), 44.9(\mathrm{~d}, J=18.1 \mathrm{~Hz}, \mathrm{C}-2), 46.5(\mathrm{~d}, J=3.3 \mathrm{~Hz}, \mathrm{C}-4), 49.1$ $\left(\mathrm{d}, J=4.2 \mathrm{~Hz}, \mathrm{C}-4^{\prime}\right), 49.9\left(\mathrm{~d}, J=18.1 \mathrm{~Hz}, \mathrm{C}-2^{\prime}\right), 64.2\left(\mathrm{~m}, \mathrm{CH}_{2} \mathrm{CH}_{3}\right), 64.7\left(\mathrm{~m}, \mathrm{CH}_{2} \mathrm{CH}_{3}\right), 171.2\left(\mathrm{C}(\mathrm{O}) \mathrm{CH}_{3}\right)$, $171.6\left(\mathrm{C}(\mathrm{O})^{\prime} \mathrm{CH}_{3}\right)$, two rotamers; ${ }^{31} \mathrm{P}$ NMR $\left(202.46 \mathrm{MHz}, \mathrm{CDCl}_{3}\right) \delta 11.70(\mathrm{~d}, J=70.2 \mathrm{~Hz}), 11.90$ $(\mathrm{d}, J=77.2 \mathrm{~Hz})$, two rotamers; ${ }^{19} \mathrm{~F}$ NMR $\left(470.59 \mathrm{MHz}, \mathrm{CDCl}_{3}\right) \delta-192.46(\mathrm{t}, J=77.5 \mathrm{~Hz}),-192.81$ $(\mathrm{t}, J=71.4 \mathrm{~Hz})$, two rotamers. HRMS (ESI) calcd. for $\left(\mathrm{C}_{16} \mathrm{H}_{34} \mathrm{O}_{7} \mathrm{NFP}_{2} \mathrm{Na}\right)[\mathrm{M}+\mathrm{Na}]^{+} 456.1692$; found 456.1700 .

Tetraethyl 2-(N-n-pentylacetamido)ethyl-1-fluoro-1,1-bisphosphonate (77): 75\% yield; colorless oil; ${ }^{1} \mathrm{H}$ NMR $\left(500.13 \mathrm{MHz} \mathrm{CDCl}_{3}\right) \delta 0.89(\mathrm{t}, J=7.5 \mathrm{~Hz}, 3 \mathrm{H}, \mathrm{H}-8), 0.90\left(\mathrm{t}, J=7.5 \mathrm{~Hz}, 3 \mathrm{H}, \mathrm{H}-8^{\prime}\right), 1.24\left(\mathrm{~m}, 2 \mathrm{H}, \mathrm{CH}_{2}\right)$, $1.37\left(\mathrm{t}, J=6.7 \mathrm{~Hz}, 12 \mathrm{H}, \mathrm{CH}_{2} \mathrm{CH}_{3}\right), 1.38\left(\mathrm{dt}, J=6.5,2.6 \mathrm{~Hz}, 12 \mathrm{H}, \mathrm{CH}_{2} \mathrm{CH}_{3}^{\prime}\right), 1.51(\mathrm{p}, J=7.6 \mathrm{~Hz}, 2 \mathrm{H}, \mathrm{H}-5)$, $1.55\left(\mathrm{p}, J=7.7 \mathrm{~Hz}, 2 \mathrm{H}, \mathrm{H}-5^{\prime}\right), 2.12\left(\mathrm{~s}, 3 \mathrm{H}, \mathrm{C}(\mathrm{O}) \mathrm{CH}_{3}\right), 2.20\left(\mathrm{~s}, 3 \mathrm{H}, \mathrm{C}(\mathrm{O}) \mathrm{CH}_{3}\right), 3.47(\mathrm{t}, J=7.9 \mathrm{~Hz}, 2 \mathrm{H}, \mathrm{H}-4)$, $4.10(\mathrm{t}, J=9.6 \mathrm{~Hz}, 2 \mathrm{H}, \mathrm{H}-2), 4.15\left(\mathrm{t}, J=9.1 \mathrm{~Hz}, 2 \mathrm{H}, \mathrm{H}-2^{\prime}\right), 4.27\left(\mathrm{~m}, 8 \mathrm{H}, \mathrm{CH}_{2} \mathrm{CH}_{3}\right)$, two rotamers; ${ }^{13} \mathrm{C}$ NMR (125.77 MHz, $\left.\mathrm{CDCl}_{3}\right) \delta 13.86(\mathrm{C}-8), 13.90\left(\mathrm{C}-8^{\prime}\right), 16.36\left(\mathrm{~m}, \mathrm{CH}_{2} \mathrm{CH}_{3}\right), 21.4\left(\mathrm{C}(\mathrm{O}) \mathrm{CH}_{3}\right), 21.88(\mathrm{~d}$. $\left.J=3.5 \mathrm{~Hz}, \mathrm{C}(\mathrm{O}) \mathrm{CH}_{3}^{\prime}\right), 22.3(\mathrm{C}-7), 22.4\left(\mathrm{C}-7^{\prime}\right), 26.1(\mathrm{C}-6), 27.6\left(\mathrm{C}-6^{\prime}\right), 28.8(\mathrm{C}-5), 29.0\left(\mathrm{C}-5^{\prime}\right), 44.8(\mathrm{~d}$, $J=18.5 \mathrm{~Hz}, \mathrm{C}-2), 46.7(\mathrm{~d}, J=3.3 \mathrm{~Hz}, \mathrm{C}-4), 49.2\left(\mathrm{~d}, J=4.2 \mathrm{~Hz}, \mathrm{C}-4^{\prime}\right), 49.8\left(\mathrm{~d}, J=18.2 \mathrm{~Hz}, \mathrm{C}-2^{\prime}\right), 64.1$ $\left(\mathrm{m}, \mathrm{CH}_{2} \mathrm{CH}_{3}\right), 64.6\left(\mathrm{~m}, \mathrm{CH}_{2} \mathrm{CH}_{3}\right), 95.8(\mathrm{dt}, J=153.2,109.3 \mathrm{~Hz}, 1 \mathrm{H}, \mathrm{H}-1), 97.3(\mathrm{dt}, J=153.0,103.1 \mathrm{~Hz}$, $\left.1 \mathrm{H}, \mathrm{H}-1^{\prime}\right), 171.1\left(\mathrm{C}(\mathrm{O}) \mathrm{CH}_{3}\right), 171.4\left(\mathrm{C}(\mathrm{O})^{\prime} \mathrm{CH}_{3}\right)$, two rotamers; ${ }^{31} \mathrm{P}$ NMR $\left(202.46 \mathrm{MHz}, \mathrm{CDCl}_{3}\right) \delta 11.66$ 
$(\mathrm{d}, J=71.8 \mathrm{~Hz}), 11.87(\mathrm{~d}, J=78.0 \mathrm{~Hz})$, two rotamers; ${ }^{19} \mathrm{~F}$ NMR $\left(470.59 \mathrm{MHz}, \mathrm{CDCl}_{3}\right) \delta-192.56$ $(\mathrm{t}, J=77.4 \mathrm{~Hz}),-192.82(\mathrm{t}, J=71.2 \mathrm{~Hz})$; two rotamers.

Tetraethyl 2-(N-n-hexylacetamido)ethyl-1-fluoro-1,1-bisphosphonate (78): 36\% yield; colorless oil; ${ }^{1} \mathrm{H}$ NMR $\left(500.13 \mathrm{MHz}, \mathrm{CDCl}_{3}\right) \delta 0.87(\mathrm{t}, J=6.9 \mathrm{~Hz}, 3 \mathrm{H}, \mathrm{H}-9), 0.89\left(\mathrm{t}, J=6.9 \mathrm{~Hz}, 3 \mathrm{H}, \mathrm{H}-9^{\prime}\right), 1.29\left(\mathrm{~m}, 6 \mathrm{H}, \mathrm{CH}_{2}\right)$, $1.37\left(\mathrm{t}, J=6.9 \mathrm{~Hz}, 12 \mathrm{H}, \mathrm{CH}_{2} \mathrm{CH}_{3}\right), 1.38\left(\mathrm{dt}, J=6.9,2.7 \mathrm{~Hz}, 12 \mathrm{H}, \mathrm{CH}_{2} \mathrm{CH}_{3}^{\prime}\right), 1.49(\mathrm{p}, J=7.5 \mathrm{~Hz}, 2 \mathrm{H}, \mathrm{H}-5)$, $1.54\left(\mathrm{p}, J=7.8 \mathrm{~Hz}, 2 \mathrm{H}, \mathrm{H}-5^{\prime}\right), 2.12\left(\mathrm{~s}, 3 \mathrm{H}, \mathrm{C}(\mathrm{O}) \mathrm{CH}_{3}\right), 2.19\left(\mathrm{~s}, 3 \mathrm{H}, \mathrm{C}(\mathrm{O}) \mathrm{CH}_{3}\right), 3.46(\mathrm{t}, J=7.9 \mathrm{~Hz}, 2 \mathrm{H}, \mathrm{H}-4)$, $4.09(\mathrm{t}, J=9.4 \mathrm{~Hz}, 2 \mathrm{H}, \mathrm{H}-2), 4.09\left(\mathrm{t}, J=9.4 \mathrm{~Hz}, 2 \mathrm{H}, \mathrm{H}-2^{\prime}\right), 4.26\left(\mathrm{~m}, 8 \mathrm{H}, \mathrm{CH}_{2} \mathrm{CH}_{3}\right)$, two rotamers; ${ }^{13} \mathrm{C}$ NMR (125.77 MHz, $\left.\mathrm{CDCl}_{3}\right) \delta 13.95(\mathrm{C}-9), 14.00\left(\mathrm{C}-9^{\prime}\right), 16.39\left(\mathrm{t}, J=3.2 \mathrm{~Hz}, \mathrm{CH}_{2} \mathrm{CH}_{3}\right), 16.44$ $\left(\mathrm{t}, J=2.6 \mathrm{~Hz}, \mathrm{CH}_{2} \mathrm{CH}_{3}^{\prime}\right), 21.5\left(\mathrm{C}(\mathrm{O}) \mathrm{CH}_{3}\right), 22.0\left(\mathrm{~d}, J=3.5 \mathrm{~Hz}, \mathrm{C}(\mathrm{O}) \mathrm{CH}_{3}^{\prime}\right), 22.5(\mathrm{C}-8), 22.6\left(\mathrm{C}-8^{\prime}\right), 26.56$ (C-6), 26.58 (C-6'), $28.0(\mathrm{C}-5), 31.5(\mathrm{C}-7), 31.6\left(\mathrm{C}-7^{\prime}\right), 44.9(\mathrm{~d}, J=18.2 \mathrm{~Hz}, \mathrm{C}-2), 46.8$ (d, J = 3.2 Hz, C-4), $49.3\left(\mathrm{~d}, J=4.3 \mathrm{~Hz}, \mathrm{C}-4^{\prime}\right), 49.9\left(\mathrm{~d}, J=18.4 \mathrm{~Hz}, \mathrm{C}-2^{\prime}\right), 62.4\left(\mathrm{~d}, J=6.8 \mathrm{~Hz}, \mathrm{CH}_{2} \mathrm{CH}_{3}\right), 62.9$ $\left(\mathrm{d}, J=6.7 \mathrm{~Hz}, \mathrm{CH}_{2} \mathrm{CH}_{3}\right), 171.1\left(\mathrm{C}(\mathrm{O}) \mathrm{CH}_{3}\right), 171.5\left(\mathrm{C}(\mathrm{O})^{\prime} \mathrm{CH}_{3}\right)$, two rotamers; ${ }^{31} \mathrm{P}$ NMR $(202.46 \mathrm{MHz}$, $\left.\mathrm{CDCl}_{3}\right) \delta 11.71(\mathrm{~d}, J=69.7 \mathrm{~Hz}), 11.91(\mathrm{~d}, J=77.1 \mathrm{~Hz})$, two rotamers; ${ }^{19} \mathrm{~F}$ NMR $\left(470.59 \mathrm{MHz}, \mathrm{CDCl}_{3}\right)$ $\delta-192.50(\mathrm{t}, J=77.6 \mathrm{~Hz}),-192.78(\mathrm{t}, J=71.2 \mathrm{~Hz})$; two rotamers. HRMS (ESI) calcd. for $\left(\mathrm{C}_{18} \mathrm{H}_{39} \mathrm{O}_{7} \mathrm{NFP}_{2}\right)$ $[\mathrm{M}+\mathrm{H}]^{+}$462.2188; found 462.2197.

Tetraethyl 2-(N-n-heptylacetamido)ethyl-1-fluoro-1,1-bisphosphonate (79): 53\% yield; colorless oil; ${ }^{1} \mathrm{H}$ NMR $\left(500.13 \mathrm{MHz}, \mathrm{CDCl}_{3}\right) \delta 0.87(\mathrm{t}, J=6.4 \mathrm{~Hz}, 3 \mathrm{H}, \mathrm{H}-10), 0.88\left(\mathrm{t}, J=6.9 \mathrm{~Hz}, 3 \mathrm{H}, \mathrm{H}-10^{\prime}\right), 1.27\left(\mathrm{~m}, 8 \mathrm{H}, \mathrm{CH}_{2}\right)$, $1.36\left(\mathrm{t}, J=7.0 \mathrm{~Hz}, 12 \mathrm{H}, \mathrm{CH}_{2} \mathrm{CH}_{3}\right), 1.38\left(\mathrm{dt}, J=6.8,3.4 \mathrm{~Hz}, 12 \mathrm{H}, \mathrm{CH}_{2} \mathrm{CH}_{3}^{\prime}\right), 1.49(\mathrm{p}, J=7.6 \mathrm{~Hz}, 2 \mathrm{H}$, $\mathrm{H}-5), 1.54\left(\mathrm{p}, J=7.8 \mathrm{~Hz}, 2 \mathrm{H}, \mathrm{H}-5^{\prime}\right), 2.11\left(\mathrm{~s}, 3 \mathrm{H}, \mathrm{C}(\mathrm{O}) \mathrm{CH}_{3}\right), 2.19\left(\mathrm{~s}, 3 \mathrm{H}, \mathrm{C}(\mathrm{O}) \mathrm{CH}_{3}\right), 3.46(\mathrm{t}, J=7.9 \mathrm{~Hz}$, $2 \mathrm{H}, \mathrm{H}-4), 4.09$ (dd, $J=10.3,8.6 \mathrm{~Hz}, 2 \mathrm{H}, \mathrm{H}-2), 4.13\left(\mathrm{t}, J=10.2,8.7 \mathrm{~Hz}, 2 \mathrm{H}, \mathrm{H}-2^{\prime}\right), 4.27\left(\mathrm{~m}, 8 \mathrm{H}, \mathrm{CH}_{2} \mathrm{CH}_{3}\right)$, two rotamers; ${ }^{13} \mathrm{C}$ NMR $\left(125.77 \mathrm{MHz}, \mathrm{CDCl}_{3}\right) \delta 13.99(\mathrm{C}-10), 14.02\left(\mathrm{C}-10^{\prime}\right), 16.3\left(\mathrm{t}, J=3.2 \mathrm{~Hz}, \mathrm{CH}_{2} \mathrm{CH}_{3}\right)$, $16.4\left(\mathrm{t}, J=2.4 \mathrm{~Hz}, \mathrm{CH}_{2} \mathrm{CH}_{3}^{\prime}\right), 21.5\left(\mathrm{C}(\mathrm{O}) \mathrm{CH}_{3}\right), 21.9\left(\mathrm{~d}, J=3.5 \mathrm{~Hz}, \mathrm{C}(\mathrm{O}) \mathrm{CH}_{3}^{\prime}\right), 22.50(\mathrm{C}-9), 22.53\left(\mathrm{C}-9^{\prime}\right)$, 26.6 (C-6), $26.7\left(\mathrm{C}^{\prime} 6^{\prime}\right), 26.9$ (C-7), $28.0(\mathrm{C}-5), 31.69(\mathrm{C}-8), 31.74\left(\mathrm{C}-8^{\prime}\right), 44.9$ (d, J = 17.9 Hz, C-2), 46.8 (d, $J=3.2 \mathrm{~Hz}, \mathrm{C}-4), 49.3\left(\mathrm{~d}, J=4.3 \mathrm{~Hz}, \mathrm{C}-4^{\prime}\right), 49.9\left(\mathrm{~d}, J=18.3 \mathrm{~Hz}, \mathrm{C}-2^{\prime}\right), 64.2\left(\mathrm{~m}, \mathrm{CH}_{2} \mathrm{CH}_{3}\right), 95.9(\mathrm{dt}$, $J=153.1,102.8 \mathrm{~Hz}, 1 \mathrm{H}, \mathrm{H}-1), 97.4\left(\mathrm{dt}, J=153.1,102.8 \mathrm{~Hz}, 1 \mathrm{H}, \mathrm{H}-1^{\prime}\right), 171.2\left(\mathrm{C}(\mathrm{O}) \mathrm{CH}_{3}\right), 171.5\left(\mathrm{C}(\mathrm{O})^{\prime} \mathrm{CH}_{3}\right)$, two rotamers; ${ }^{31} \mathrm{P}$ NMR $\left(202.46 \mathrm{MHz}, \mathrm{CDCl}_{3}\right) \delta 11.69(\mathrm{~d}, J=72.1 \mathrm{~Hz}), 11.89(\mathrm{~d}, J=78.4 \mathrm{~Hz})$, two rotamers; ${ }^{19} \mathrm{~F}$ NMR $\left(470.59 \mathrm{MHz}, \mathrm{CDCl}_{3}\right) \delta-192.52(\mathrm{t}, J=77.5 \mathrm{~Hz}),-192.79(\mathrm{t}, J=71.3 \mathrm{~Hz})$; two rotamers.

Tetraethyl 2-(N-n-octylacetamido)ethyl-1-fluoro-1,1-bisphosphonate (80): $52 \%$ yield; colorless oil; ${ }^{1} \mathrm{H} N M R$ $\left(500.13 \mathrm{MHz}, \mathrm{CDCl}_{3}\right) \delta 0.87(\mathrm{t}, J=7.1 \mathrm{~Hz}, 3 \mathrm{H}, \mathrm{H}-11), 0.88\left(\mathrm{t}, J=6.9 \mathrm{~Hz}, 3 \mathrm{H}, \mathrm{H}-11^{\prime}\right), 1.27\left(\mathrm{~m}, 10 \mathrm{H}, \mathrm{CH}_{2}\right)$, $1.37\left(\mathrm{t}, J=7.0 \mathrm{~Hz}, 12 \mathrm{H}, \mathrm{CH}_{2} \mathrm{CH}_{3}\right), 1.38\left(\mathrm{dt}, J=6.8,3.4 \mathrm{~Hz}, 12 \mathrm{H}, \mathrm{CH}_{2} \mathrm{CH}_{3}^{\prime}\right), 1.50(\mathrm{p}, J=7.6 \mathrm{~Hz}, 2 \mathrm{H}$, $\mathrm{H}-5), 1.54\left(\mathrm{p}, J=7.9 \mathrm{~Hz}, 2 \mathrm{H}, \mathrm{H}-5^{\prime}\right), 2.12\left(\mathrm{~s}, 3 \mathrm{H}, \mathrm{C}(\mathrm{O}) \mathrm{CH}_{3}\right), 2.19\left(\mathrm{~s}, 3 \mathrm{H}, \mathrm{C}(\mathrm{O}) \mathrm{CH}_{3}\right), 3.46(\mathrm{t}, J=8.2 \mathrm{~Hz}$, $2 \mathrm{H}, \mathrm{H}-4), 4.09$ (dd, $J=12.0,7.2 \mathrm{~Hz}, 2 \mathrm{H}, \mathrm{H}-2), 4.15\left(\mathrm{t}, J=10.2,8.8 \mathrm{~Hz}, 2 \mathrm{H}, \mathrm{H}-2^{\prime}\right), 4.27\left(\mathrm{~m}, 8 \mathrm{H}, \mathrm{CH}_{2} \mathrm{CH}_{3}\right)$, two rotamers; ${ }^{13} \mathrm{C}$ NMR $\left(125.77 \mathrm{MHz}, \mathrm{CDCl}_{3}\right) \delta 14.01(\mathrm{C}-11), 14.02\left(\mathrm{C}-11^{\prime}\right), 16.4\left(\mathrm{~m}, \mathrm{CH}_{2} \mathrm{CH}_{3}\right), 21.5$ $\left(\mathrm{C}(\mathrm{O}) \mathrm{CH}_{3}\right), 21.9\left(\mathrm{~d}, J=3.5 \mathrm{~Hz}, \mathrm{C}(\mathrm{O}) \mathrm{CH}_{3}^{\prime}\right), 22.55(\mathrm{C}-10), 22.58\left(\mathrm{C}-10^{\prime}\right), 26.6(\mathrm{C}-6), 26.8\left(\mathrm{C}-6^{\prime}\right), 26.9(\mathrm{C}-7)$, 28.0 (C-5), 29.1 (C-8), $29.3\left(\mathrm{C}-8^{\prime}\right), 31.74(\mathrm{C}-9), 31.79\left(\mathrm{C}-9^{\prime}\right), 44.9$ (d, J = 18.0 Hz, C-2), 46.8 (d, J = 3.3 Hz, C-4), $49.3\left(\mathrm{~d}, J=4.2 \mathrm{~Hz}, \mathrm{C}-4^{\prime}\right), 49.9\left(\mathrm{~d}, J=18.4 \mathrm{~Hz}, \mathrm{C}-2^{\prime}\right), 64.2\left(\mathrm{~m}, \mathrm{CH}_{2} \mathrm{CH}_{3}\right), 95.9(\mathrm{dt}, J=153.1$, $109.2 \mathrm{~Hz}, 1 \mathrm{H}, \mathrm{H}-1), 97.4\left(\mathrm{dt}, J=153.1,103.3 \mathrm{~Hz}, 1 \mathrm{H}, \mathrm{H}-1^{\prime}\right), 171.2\left(\mathrm{C}(\mathrm{O}) \mathrm{CH}_{3}\right), 171.5\left(\mathrm{C}(\mathrm{O})^{\prime} \mathrm{CH}_{3}\right)$, two rotamers; ${ }^{31} \mathrm{P} \mathrm{NMR}\left(202.46 \mathrm{MHz}, \mathrm{CDCl}_{3}\right) \delta 11.69(\mathrm{~d}, J=72.1 \mathrm{~Hz}), 11.89(\mathrm{~d}, J=79.6 \mathrm{~Hz})$, two rotamers; ${ }^{19} \mathrm{~F} \mathrm{NMR}\left(470.59 \mathrm{MHz}, \mathrm{CDCl}_{3}\right) \delta-192.51(\mathrm{t}, J=77.5 \mathrm{~Hz}),-192.78(\mathrm{t}, J=71.3 \mathrm{~Hz})$; two rotamers.

Tetraethyl 2-(N-n-nonylacetamido)ethyl-1-fluoro-1,1-bisphosphonate (81): 59\% yield; colorless oil; ${ }^{1} \mathrm{H}$ NMR $\left(500.13 \mathrm{MHz}, \mathrm{CDCl}_{3}\right) \delta 0.875(\mathrm{t}, J=7.0 \mathrm{~Hz}, 3 \mathrm{H}, \mathrm{H}-11), 0.882\left(\mathrm{t}, J=6.9 \mathrm{~Hz}, 3 \mathrm{H}, \mathrm{H}-11^{\prime}\right), 1.26(\mathrm{~m}, 12 \mathrm{H}$, $\left.\mathrm{CH}_{2}\right), 1.37\left(\mathrm{t}, J=7.0 \mathrm{~Hz}, 12 \mathrm{H}, \mathrm{CH}_{2} \mathrm{CH}_{3}\right), 1.38\left(\mathrm{dt}, J=6.9,3.3 \mathrm{~Hz}, 12 \mathrm{H}, \mathrm{CH}_{2} \mathrm{CH}_{3}^{\prime}\right), 1.50(\mathrm{p}, J=7.6 \mathrm{~Hz}, 2 \mathrm{H}$, $\mathrm{H}-5), 1.54\left(\mathrm{p}, J=7.7 \mathrm{~Hz}, 2 \mathrm{H}, \mathrm{H}-5^{\prime}\right), 2.12\left(\mathrm{~s}, 3 \mathrm{H}, \mathrm{C}(\mathrm{O}) \mathrm{CH}_{3}\right), 2.19\left(\mathrm{~s}, 3 \mathrm{H}, \mathrm{C}(\mathrm{O}) \mathrm{CH}_{3}\right), 3.46(\mathrm{t}, J=7.9 \mathrm{~Hz}, 2 \mathrm{H}$, $\mathrm{H}-4), 3.48\left(\mathrm{t}, J=8.5 \mathrm{~Hz}, 2 \mathrm{H}, \mathrm{H}-4^{\prime}\right), 4.09(\mathrm{dd}, J=10.0,8.9 \mathrm{~Hz}, 2 \mathrm{H}, \mathrm{H}-2), 4.15\left(\mathrm{t}, J=10.2,8.8 \mathrm{~Hz}, 2 \mathrm{H}, \mathrm{H}-2^{\prime}\right)$, $4.27\left(\mathrm{~m}, 8 \mathrm{H}, \mathrm{CH}_{2} \mathrm{CH}_{3}\right)$, two rotamers; ${ }^{13} \mathrm{C}$ NMR $\left(125.77 \mathrm{MHz}, \mathrm{CDCl}_{3}\right) \delta 14.0(\mathrm{C}-12), 14.1\left(\mathrm{C}-12^{\prime}\right), 16.4$ $\left(\mathrm{m}, \mathrm{CH}_{2} \mathrm{CH}_{3}\right), 21.5\left(\mathrm{C}(\mathrm{O}) \mathrm{CH}_{3}\right), 21.9\left(\mathrm{~d}, J=3.6 \mathrm{~Hz}, \mathrm{C}(\mathrm{O}) \mathrm{CH}_{3}^{\prime}\right), 22.59(\mathrm{C}-11), 22.61\left(\mathrm{C}-11^{\prime}\right), 26.6(\mathrm{C}-6), 26.8$ (C-6'), 26.9 (C-7), 28.0 (C-5), $29.16(\mathrm{C}-8), 29.21\left(\mathrm{C}-8^{\prime}\right), 29.28\left(\mathrm{C}-7^{\prime}\right) 29.39\left(\mathrm{C}-5^{\prime}\right), 29.46(\mathrm{C}-9), 29.50\left(\mathrm{C}-9^{\prime}\right)$, $31.78(\mathrm{C}-10), 31.81\left(\mathrm{C}-10^{\prime}\right), 44.9(\mathrm{~d}, J=17.9 \mathrm{~Hz}, \mathrm{C}-2), 46.8(\mathrm{~d}, J=3.4 \mathrm{~Hz}, \mathrm{C}-4), 49.3\left(\mathrm{~d}, J=4.3 \mathrm{~Hz}, \mathrm{C}-4^{\prime}\right)$, $49.9\left(\mathrm{~d}, J=18.2 \mathrm{~Hz}, \mathrm{C}-2^{\prime}\right), 64.2\left(\mathrm{~m}, \mathrm{CH}_{2} \mathrm{CH}_{3}\right), 64.7\left(\mathrm{t}, J=3.2 \mathrm{~Hz}, \mathrm{CH}_{2} \mathrm{CH}_{3}\right), 95.9(\mathrm{dt}, J=153.0,109.0 \mathrm{~Hz}$, $1 \mathrm{H}, \mathrm{H}-1), 97.4\left(\mathrm{dt}, J=153.0,103.0 \mathrm{~Hz}, 1 \mathrm{H}, \mathrm{H}-1^{\prime}\right), 171.2\left(\mathrm{C}(\mathrm{O}) \mathrm{CH}_{3}\right), 171.5\left(\mathrm{C}(\mathrm{O})^{\prime} \mathrm{CH}_{3}\right)$, two rotamers; 
${ }^{31} \mathrm{P}$ NMR $\left(202.46 \mathrm{MHz}, \mathrm{CDCl}_{3}\right) \delta 11.70(\mathrm{~d}, J=77.9 \mathrm{~Hz}), 11.91(\mathrm{~d}, J=77.9 \mathrm{~Hz})$, two rotamers; ${ }^{19} \mathrm{~F}$ NMR $\left(470.59 \mathrm{MHz}, \mathrm{CDCl}_{3}\right) \delta-192.50(\mathrm{t}, J=77.4 \mathrm{~Hz}),-192.77(\mathrm{t}, J=71.3 \mathrm{~Hz})$; two rotamers.

Tetraethyl 2-(N-n-decylacetamido)ethyl-1-fluoro-1,1-bisphosphonate (82): $54 \%$ yield; colorless oil; ${ }^{1} \mathrm{H}$ NMR $\left(500.13 \mathrm{MHz}, \mathrm{CDCl}_{3}\right) \delta 0.877(\mathrm{t}, J=7.0 \mathrm{~Hz}, 3 \mathrm{H}, \mathrm{H}-13), 0.881\left(\mathrm{t}, J=6.8 \mathrm{~Hz}, 3 \mathrm{H}, \mathrm{H}-13^{\prime}\right), 1.26(\mathrm{~m}, 14 \mathrm{H}$, $\left.\mathrm{CH}_{2}\right), 1.37\left(\mathrm{t}, J=7.0 \mathrm{~Hz}, 12 \mathrm{H}, \mathrm{CH}_{2} \mathrm{CH}_{3}\right), 1.38\left(\mathrm{dt}, J=6.9,3.1 \mathrm{~Hz}, 12 \mathrm{H}, \mathrm{CH}_{2} \mathrm{CH}_{3}^{\prime}\right), 1.50(\mathrm{p}, J=7.3 \mathrm{~Hz}, 2 \mathrm{H}$, $\mathrm{H}-5), 1.54\left(\mathrm{p}, J=7.6 \mathrm{~Hz}, 2 \mathrm{H}, \mathrm{H}-5^{\prime}\right), 2.12\left(\mathrm{~s}, 3 \mathrm{H}, \mathrm{C}(\mathrm{O}) \mathrm{CH}_{3}\right), 2.19\left(\mathrm{~s}, 3 \mathrm{H}, \mathrm{C}(\mathrm{O}) \mathrm{CH}_{3}\right), 3.46(\mathrm{t}, J=7.9 \mathrm{~Hz}, 2 \mathrm{H}$, $\mathrm{H}-4), 3.48\left(\mathrm{t}, J=8.2 \mathrm{~Hz}, 2 \mathrm{H}, \mathrm{H}-4^{\prime}\right), 4.09(\mathrm{dd}, J=10.4,8.6 \mathrm{~Hz}, 2 \mathrm{H}, \mathrm{H}-2), 4.15\left(\mathrm{t}, J=10.1,8.8 \mathrm{~Hz}, 2 \mathrm{H}, \mathrm{H}-2^{\prime}\right)$, $4.27\left(\mathrm{~m}, 8 \mathrm{H}, \mathrm{CH}_{2} \mathrm{CH}_{3}\right)$, two rotamers; ${ }^{13} \mathrm{C}$ NMR $\left(125.77 \mathrm{MHz}, \mathrm{CDCl}_{3}\right) \delta 14.0(\mathrm{C}-13), 16.4\left(\mathrm{~m}, \mathrm{CH}_{2} \mathrm{CH}_{3}\right)$, $21.5\left(\mathrm{C}(\mathrm{O}) \mathrm{CH}_{3}\right), 21.9\left(\mathrm{~d}, J=3.9 \mathrm{~Hz}, \mathrm{C}(\mathrm{O}) \mathrm{CH}_{3}^{\prime}\right), 22.61(\mathrm{C}-12), 22.61\left(\mathrm{C}-12^{\prime}\right), 26.6(\mathrm{C}-6), 26.8\left(\mathrm{C}-6^{\prime}\right), 26.9$ (C-7), 28.0 (C-5), 29.23 (C-8), 29.25 (C-8'), 29.27 (C-7') 29.39 (C-5'), 29.45 (C-9), 29.50 (C-10), 29.54 (C-10'), $31.81(\mathrm{C}-11), 31.83\left(\mathrm{C}-11^{\prime}\right), 44.9(\mathrm{~d}, J=18.6 \mathrm{~Hz}, \mathrm{C}-2), 46.8(\mathrm{~d}, J=3.4 \mathrm{~Hz}, \mathrm{C}-4), 49.3\left(\mathrm{~d}, J=4.5 \mathrm{~Hz}, \mathrm{C}-4^{\prime}\right)$, $49.9\left(\mathrm{~d}, J=18.4 \mathrm{~Hz}, \mathrm{C}-2^{\prime}\right), 64.2\left(\mathrm{~m}, \mathrm{CH}_{2} \mathrm{CH}_{3}\right), 64.7\left(\mathrm{t}, J=3.2 \mathrm{~Hz}, \mathrm{CH}_{2} \mathrm{CH}_{3}\right), 95.9(\mathrm{dt}, J=153.0,109.0 \mathrm{~Hz}$, $1 \mathrm{H}, \mathrm{H}-1), 97.4\left(\mathrm{dt}, J=153.0,103.1 \mathrm{~Hz}, 1 \mathrm{H}, \mathrm{H}-1^{\prime}\right), 171.2\left(\mathrm{C}(\mathrm{O}) \mathrm{CH}_{3}\right), 171.5\left(\mathrm{C}(\mathrm{O})^{\prime} \mathrm{CH}_{3}\right)$, two rotamers; ${ }^{31} \mathrm{P} \mathrm{NMR}\left(202.46 \mathrm{MHz}, \mathrm{CDCl}_{3}\right) \delta 11.70(\mathrm{~d}, J=71.2 \mathrm{~Hz}), 11.90(\mathrm{~d}, J=77.7 \mathrm{~Hz})$, two rotamers; ${ }^{19} \mathrm{~F}$ NMR $\left(470.59 \mathrm{MHz}, \mathrm{CDCl}_{3}\right) \delta-192.50(\mathrm{t}, J=77.5 \mathrm{~Hz}),-192.78(\mathrm{t}, J=71.2 \mathrm{~Hz})$; two rotamers.

Hydrolysys. General Procedure. Esters 75-82 were treated, in independent experiments, with a concentrated aqueous solution of hydrochloric acid $(2.0 \mathrm{~mL})$. The resulting mixtures were refluxed for $24 \mathrm{~h}$. The solvent was evaporated and the residues were crystallized from water-ethanol.

1-[(n-Prop-1-ylamino)ethyl]-1-fluoro-1,1-bisphosphonic Acid (54): 54\% yield; white solid; m.p. $161{ }^{\circ} \mathrm{C}$; ${ }^{1} \mathrm{H}$ NMR (500.13 MHz, $\left.\mathrm{D}_{2} \mathrm{O}\right) \delta 0.91(\mathrm{t}, J=7.5 \mathrm{~Hz}, 3 \mathrm{H}, \mathrm{H}-6), 1.65$ (sext, $\left.J=7.4 \mathrm{~Hz}, 2 \mathrm{H}, \mathrm{H}-5\right), 3.04$ $(\mathrm{t}, J=7.3 \mathrm{~Hz}, 2 \mathrm{H}, \mathrm{H}-4), 3.60$ (m, 2H, H-2); ${ }^{13} \mathrm{C}$ NMR (125.77 MHz, $\left.\mathrm{D}_{2} \mathrm{O}\right) \delta 10.4$ (C-6), 19.5 (C-5), 49.6 $(\mathrm{d}, J=16.1, \mathrm{C}-2), 50.2(\mathrm{C}-4) ;{ }^{31} \mathrm{P}$ NMR $\left(202.46 \mathrm{MHz}, \mathrm{D}_{2} \mathrm{O}\right) \delta 8.89(\mathrm{~d}, J=65.4 \mathrm{~Hz}) ;{ }^{19} \mathrm{~F}$ NMR $(470.59$ $\left.\mathrm{MHz}, \mathrm{D}_{2} \mathrm{O}\right) \delta-193.32(\mathrm{t}, J=65.5 \mathrm{~Hz})$. HRMS (ESI) calcd. for $\left(\mathrm{C}_{5} \mathrm{H}_{15} \mathrm{O}_{6} \mathrm{NFP}_{2}\right)[\mathrm{M}+\mathrm{H}]^{+}$266.0359; found 266.0359 .

1-[(n-But-1-ylamino)ethyl]-1-fluoro-1,1-bisphosphonic Acid (55): 70\% yield; white solid; m.p. $153-154{ }^{\circ} \mathrm{C}$; ${ }^{1} \mathrm{H}$ NMR $\left(500.13 \mathrm{MHz}, \mathrm{D}_{2} \mathrm{O}\right) \delta 0.85(\mathrm{t}, J=7.4 \mathrm{~Hz}, 3 \mathrm{H}, \mathrm{H}-7), 1.32$ (sext, $\left.J=7.5 \mathrm{~Hz}, 2 \mathrm{H}, \mathrm{H}-6\right), 1.61$ $(\mathrm{p}, J=7.5 \mathrm{~Hz}, 2 \mathrm{H}, \mathrm{H}-5), 3.07$ (t, $J=7.4 \mathrm{~Hz}, 2 \mathrm{H}, \mathrm{H}-4), 3.59(\mathrm{~m}, 2 \mathrm{H}, \mathrm{H}-2) ;{ }^{13} \mathrm{C}$ NMR $\left(125.77 \mathrm{MHz}, \mathrm{D}_{2} \mathrm{O}\right) \delta$ 12.7 (C-7), 19.0 (C-6), 27.5 (C-5), 48.1 (C-4), 49.1 (dt, $J=18.1,2.3 \mathrm{~Hz}, \mathrm{C}-2) ;{ }^{31} \mathrm{P}$ NMR (202.46 MHz, $\left.\mathrm{D}_{2} \mathrm{O}\right)$ $\delta 8.84(\mathrm{~d}, J=65.5 \mathrm{~Hz}) ;{ }^{19} \mathrm{~F}$ NMR $\left(470.59 \mathrm{MHz}, \mathrm{D}_{2} \mathrm{O}\right) \delta-193.39(\mathrm{t}, J=65.6 \mathrm{~Hz})$. HRMS (ESI) calcd. for $\left(\mathrm{C}_{6} \mathrm{H}_{17} \mathrm{O}_{6} \mathrm{NFP}_{2}\right)[\mathrm{M}+\mathrm{H}]^{+}$280.0515; found 280.0514.

1-[(n-Pent-1-ylamino)ethyl]-1-fluoro-1,1-bisphosphonic Acid (56): 65\% yield; white solid; m.p. $164-165{ }^{\circ} \mathrm{C}$; ${ }^{1} \mathrm{H}$ NMR $\left(500.13 \mathrm{MHz}, \mathrm{D}_{2} \mathrm{O}\right) \delta 0.81(\mathrm{t}, J=7.1 \mathrm{~Hz}, 3 \mathrm{H}, \mathrm{H}-8), 1.28\left(\mathrm{~m}, 4 \mathrm{H}, \mathrm{CH}_{2}\right), 1.63(\mathrm{p}, J=7.3 \mathrm{~Hz}, 2 \mathrm{H}$, $\mathrm{H}-5), 3.06$ (t, $J=7.4 \mathrm{~Hz}, 2 \mathrm{H}, \mathrm{H}-4), 3.59$ (p, $J=10.1 \mathrm{~Hz}, 2 \mathrm{H}, \mathrm{H}-2) ;{ }^{13} \mathrm{C} \mathrm{NMR}\left(125.77 \mathrm{MHz}, \mathrm{D}_{2} \mathrm{O}\right) \delta 13.0$ (C-8), 21.4 (C-7), 25.1 (C-5), 27.7 (C-7), 48.3 (C-4), 49.1 (d, $J=18.3 \mathrm{~Hz}, \mathrm{C}-2) ;{ }^{31} \mathrm{P}$ NMR (202.46 MHz, $\left.\mathrm{D}_{2} \mathrm{O}\right)$ $\delta 8.93(\mathrm{~d}, J=47.3 \mathrm{~Hz}) ;{ }^{19} \mathrm{~F}$ NMR $\left(470.59 \mathrm{MHz}, \mathrm{D}_{2} \mathrm{O}\right) \delta-193.32(\mathrm{t}, J=65.5 \mathrm{~Hz})$. HRMS (ESI) calcd. for $\left(\mathrm{C}_{7} \mathrm{H}_{19} \mathrm{O}_{6} \mathrm{NFP}_{2}\right)[\mathrm{M}+\mathrm{H}]^{+}$294.0672; found 294.0667.

1-[(n-Hex-1-ylamino)ethyl]-1-fluoro-1,1-bisphosphonic Acid (57): 34\% yield; white solid; ${ }^{1} \mathrm{H}$ NMR $\left(500.13 \mathrm{MHz}, \mathrm{D}_{2} \mathrm{O}\right) \delta 0.77(\mathrm{t}, J=7.1 \mathrm{~Hz}, 3 \mathrm{H}, \mathrm{H}-9), 1.22\left(\mathrm{~m}, 4 \mathrm{H}, \mathrm{CH}_{2}\right), 1.28\left(\mathrm{~m}, 2 \mathrm{H}, \mathrm{CH}_{2}\right), 1.61$ $(\mathrm{p}, J=7.5 \mathrm{~Hz}, 2 \mathrm{H}, \mathrm{H}-5), 3.05(\mathrm{t}, J=7.5 \mathrm{~Hz}, 2 \mathrm{H}, \mathrm{H}-4), 3.58(\mathrm{p}, J=10.6 \mathrm{~Hz}, 2 \mathrm{H}, \mathrm{H}-2) ;{ }^{13} \mathrm{C}$ NMR (125.77 MHz, $\left.\mathrm{D}_{2} \mathrm{O}\right) \delta 13.1$ (C-9), 21.6 (C-8), 25.2 (C-6), 25.4 (C-5), 30.3 (C-7), 49.1 (dt, J = 18.1, 2.3 Hz, C-2), $92.2(\mathrm{dt}, J=184.1,139.4 \mathrm{~Hz}, \mathrm{C}-1) ;{ }^{31} \mathrm{P}$ NMR $\left(202.46 \mathrm{MHz}, \mathrm{D}_{2} \mathrm{O}\right) \delta 8.91(\mathrm{~d}, J=59.7 \mathrm{~Hz}) ;{ }^{19} \mathrm{~F} \mathrm{NMR}$ $\left(470.59 \mathrm{MHz}, \mathrm{D}_{2} \mathrm{O}\right) \delta-193.54(\mathrm{t}, J=65.9 \mathrm{~Hz})$. HRMS (ESI) calcd. for $\left(\mathrm{C}_{8} \mathrm{H}_{21} \mathrm{O}_{6} \mathrm{NFP}_{2}\right)[\mathrm{M}+\mathrm{H}]^{+} 308.0823$; found 308.0822 .

1-[(n-Hept-1-ylamino)ethyl]-1-fluoro-1,1-bisphosphonic Acid (58): 56\% yield; white solid; m.p. $135-136{ }^{\circ} \mathrm{C}$; ${ }^{1} \mathrm{H}$ NMR (500.13 MHz, $\left.\mathrm{D}_{2} \mathrm{O}\right) \delta 0.76(\mathrm{t}, J=7.1 \mathrm{~Hz}, 3 \mathrm{H}, \mathrm{H}-10), 1.20\left(\mathrm{~m}, 4 \mathrm{H}, \mathrm{CH}_{2}\right), 1.27\left(\mathrm{~m}, 4 \mathrm{H}, \mathrm{CH}_{2}\right), 1.61$ $(\mathrm{p}, J=7.4 \mathrm{~Hz}, 2 \mathrm{H}, \mathrm{H}-5), 3.06(\mathrm{t}, J=7.7 \mathrm{~Hz}, 2 \mathrm{H}, \mathrm{H}-4), 3.59$ (p, $J=10.7 \mathrm{~Hz}, 2 \mathrm{H}, \mathrm{H}-2) ;{ }^{13} \mathrm{C}$ NMR (125.77 MHz, $\left.\mathrm{D}_{2} \mathrm{O}\right) \delta 13.2$ (C-10), 21.8 (C-9), 25.4 (C-6), 25.5 (C-7), 27.8 (C-5), 30.7 (C-8), 48.4 (C-4), $49.0(\mathrm{dt}, J=17.9,2.3 \mathrm{~Hz}, \mathrm{C}-2), 92.1(\mathrm{dt}, J=184.2 \mathrm{~Hz}, 139.6 \mathrm{~Hz}, \mathrm{C}-1) ;{ }^{31} \mathrm{P}$ NMR $\left(202.46 \mathrm{MHz}, \mathrm{CDCl}_{3}\right)$ 
$\delta 8.90(\mathrm{~d}, J=60.8 \mathrm{~Hz}) ;{ }^{19} \mathrm{~F}$ NMR $\left(470.59 \mathrm{MHz}, \mathrm{D}_{2} \mathrm{O}\right)-193.65(\mathrm{t}, J=66.0 \mathrm{~Hz})$. HRMS (ESI) calcd. for $\left(\mathrm{C}_{9} \mathrm{H}_{23} \mathrm{O}_{6} \mathrm{NFP}_{2}\right)[\mathrm{M}+\mathrm{H}]^{+}$322.0985; found 322.0969.

1-[(n-Oct-1-ylamino)ethyl]-1-fluoro-1,1-bisphosphonic Acid (59): 31\% yield; white solid; m.p. $>270{ }^{\circ} \mathrm{C} ;{ }^{1} \mathrm{H}$ NMR (500.13 MHz, $\left.\mathrm{D}_{2} \mathrm{O}\right) \delta 0.74(\mathrm{t}, J=6.9 \mathrm{~Hz}, 3 \mathrm{H}, \mathrm{H}-11), 1.19\left(\mathrm{~m}, 10 \mathrm{H}, \mathrm{CH}_{2}\right), 1.59(\mathrm{p}, J=7.4 \mathrm{~Hz}, 2 \mathrm{H}$, $\mathrm{H}-5), 3.02$ (t, $J=7.6 \mathrm{~Hz}, 2 \mathrm{H}, \mathrm{H}-4), 3.57$ (p, $J=10.2 \mathrm{~Hz}, 2 \mathrm{H}, \mathrm{H}-2) ;{ }^{13} \mathrm{C}$ NMR $\left(125.77 \mathrm{MHz}, \mathrm{D}_{2} \mathrm{O}\right) \delta 13.4$ (C-11), 22.0 (C-10), 25.4 (C-6), 25.6 (C-7), 28.1 (C-8), 28.2 (C-5), 31.0 (C-9), 48.2 (C-4), 48.9 (d, J = 18.1 Hz, C-2), $92.1(\mathrm{dt}, J=184.6 \mathrm{~Hz}, 139.8 \mathrm{~Hz}, \mathrm{C}-1) ;{ }^{31} \mathrm{P} \mathrm{NMR}\left(202.46 \mathrm{MHz}, \mathrm{CDCl}_{3}\right) \delta 8.87 \mathrm{ppm}(\mathrm{d}, J=61.0 \mathrm{~Hz})$; ${ }^{19} \mathrm{~F}$ NMR $\left(470.59 \mathrm{MHz}, \mathrm{D}_{2} \mathrm{O}\right) \delta-193.71(\mathrm{t}, J=66.1 \mathrm{~Hz})$.

1-[(n-Non-1-ylamino)ethyl]-1-fluoro-1,1-bisphosphonic Acid (60). White solid; m.p. $147{ }^{\circ} \mathrm{C} ;{ }^{1} \mathrm{H}$ NMR $\left(500.13 \mathrm{MHz}, \mathrm{D}_{2} \mathrm{O}\right) \delta 0.77(\mathrm{t}, J=7.1 \mathrm{~Hz}, 3 \mathrm{H}, \mathrm{H}-12), 1.19\left(\mathrm{~m}, 12 \mathrm{H}, \mathrm{CH}_{2}\right), 1.62(\mathrm{p}, J=6.67 \mathrm{~Hz}, 2 \mathrm{H}, \mathrm{H}-5)$, $3.06(\mathrm{t}, J=7.1 \mathrm{~Hz}, 2 \mathrm{H}, \mathrm{H}-4), 3.59(\mathrm{~m}, 2 \mathrm{H}, \mathrm{H}-2) ;{ }^{13} \mathrm{C}$ NMR $\left(125.77 \mathrm{MHz}, \mathrm{D}_{2} \mathrm{O}\right) \delta 13.4(\mathrm{C}-12), 22.0(\mathrm{C}-11)$, 25.4 (C-6), 25.5 (C-7), 28.1 (C-9), 28.3 (C-8), 28.4 (C-5), 31.1 (C-10), 48.4 (C-4), 49.1 (d, J = 18.1 Hz, C-2), $92.3(\mathrm{dt}, J=184.9 \mathrm{~Hz}, 139.4 \mathrm{~Hz}, \mathrm{C}-1) ;{ }^{31} \mathrm{P}$ NMR $\left(202.46 \mathrm{MHz}, \mathrm{CDCl}_{3}\right) \delta 8.83 \mathrm{ppm}(\mathrm{d}, J=65.7 \mathrm{~Hz}) ;{ }^{19} \mathrm{~F}$ NMR $\left(470.59 \mathrm{MHz}, \mathrm{D}_{2} \mathrm{O}\right) \delta-193.34(\mathrm{t}, J=65.6 \mathrm{~Hz})$. HRMS (ESI) calcd. for $\left(\mathrm{C}_{11} \mathrm{H}_{27} \mathrm{O}_{6} \mathrm{NFP}_{2}\right)[\mathrm{M}+\mathrm{H}]^{+}$ 350.1298; found 350.1306 .

1-[(n-Dec-1-ylamino)ethyl]-1-fluoro-1,1-bisphosphonic Acid (61): 54\% yield; White solid; m.p. $151{ }^{\circ} \mathrm{C}$; ${ }^{1} \mathrm{H}$ NMR $\left(500.13 \mathrm{MHz}, \mathrm{D}_{2} \mathrm{O}\right) \delta 0.78(\mathrm{t}, J=6.7 \mathrm{~Hz}, 3 \mathrm{H}, \mathrm{H}-13), 1.20\left(\mathrm{~m}, 10 \mathrm{H}, \mathrm{CH}_{2}\right), 1.27\left(\mathrm{~m}, 4 \mathrm{H}, \mathrm{CH}_{2}\right), 1.64$ $(\mathrm{p}, J=7.3 \mathrm{~Hz}, 2 \mathrm{H}, \mathrm{H}-5), 3.06(\mathrm{t}, J=7.0 \mathrm{~Hz}, 2 \mathrm{H}, \mathrm{H}-4), 3.62(\mathrm{~m}, 2 \mathrm{H}, \mathrm{H}-2) ;{ }^{13} \mathrm{C}$ NMR $\left(125.77 \mathrm{MHz}, \mathrm{D}_{2} \mathrm{O}\right) \delta$ 13.4 (C-13), 22.0 (C-12), 25.4 (C-6), 25.5 (C-7), 28.1 (C-10), 28.4 (C-8,C-9), 28.6 (C-5), 31.1 (C-11), 48.4 (C-4), $49.1(\mathrm{dt}, J=18.1,2.3 \mathrm{~Hz}, \mathrm{C}-2) ;{ }^{31} \mathrm{P}$ NMR $\left(202.46 \mathrm{MHz}, \mathrm{CDCl}_{3}\right) \delta 8.75 \mathrm{ppm}(\mathrm{d}, J=65.6 \mathrm{~Hz}) ;{ }^{19} \mathrm{~F}$ NMR $\left(470.59 \mathrm{MHz}, \mathrm{D}_{2} \mathrm{O}\right) \delta-199.16(\mathrm{t}, J=65.8 \mathrm{~Hz})$. HRMS (ESI) calcd. for $\left(\mathrm{C}_{12} \mathrm{H}_{23} \mathrm{O}_{6} \mathrm{NFP}_{2}\right)[\mathrm{M}+\mathrm{H}]^{+}$ 364.1454; found 364.1443.

\section{Drug Screening}

\subsection{T. cruzi Amastigote Assays}

These experiments were done as reported using tdTomato labeled trypomastigotes with the modifications described by Recher et al., 2013 [38]. ED $_{50}$ values were determined by non-linear regression analysis using SigmaPlot.

\subsection{T. gondii Tachyzoites Assays}

Experiments on $T$. gondii tachyzoites were carried out as described previously using $T$. gondii tachyzoites expressing red fluorescent protein with the modifications described by Recher et al., 2013 [38]. Plates were read with covered lids, and both excitation $(544 \mathrm{~nm})$ and emission $(590 \mathrm{~nm})$ were read from the bottom.

\subsection{Cytotoxicity for Vero cells}

The cytotoxicity was tested using the Alamar Blue ${ }^{\mathrm{TM}}$ assay as described by Recher et al., 2013 [38].

\subsection{TcFPPS and TgFPPS assays and product analysis}

The enzymatic activity of the target enzymes was performed according to our previous studies as described for Szajnman et al., 2008 [34].

\section{Conclusions}

In this paper, we have described the synthesis and biological evaluation of isoprenoid and linear 1,1-bisphosphonates against $T$. cruzi and T. gondii cells as well as the target enzymes TcFPPS and TgFPPS. The title compounds exhibited marginal antiparasitic activity. However, the biological data here presented provide insights concerning structure-activity relationship. Clearly, the simple replacement of the hydrogen atom by a fluorine atom had a dramatic effect on the biological activity, giving rise to inactive compounds. 
Supplementary Materials: Copies of the ${ }^{1} \mathrm{H}$ NMR, ${ }^{13} \mathrm{C}$ NMR, ${ }^{31} \mathrm{P}$ NMR and ${ }^{19} \mathrm{~F}$ NMR spectra of the target molecules and the corresponding intermediates are included as supporting information. They are available online at http://www.mdpi.com/1420-3049/22/1/82/s1.

Acknowledgments: This work was supported by grants from the Consejo Nacional de Investigaciones Científicas y Técnicas (PIP 0797), the Agencia Nacional de Promoción Científica y Tecnológica (PICT 2012 \#0457), and the Universidad de Buenos Aires (20020130100223BA) to J.B.R., and the U.S. National Institutes of Health to R.D. (AI-107663) and S.N.J.M. (AI-102254).

Author Contributions: Tamila Galaka and Mariana Ferrer Casal performed the synthetic aspects of the experiment and wrote the draft of paper. Sergio H. Szajnman supervised the synthetic aspects of this work. Melissa Storey, Catherine Li and María N. Chao performed biological assays. Roberto Docampo and Silvia N. J. Moreno supervised the biological aspects of this study. Juan B. Rodriguez participated in the writing and revision of the manuscript. All authors took part in data analysis and discussion. All authors read and approved the final manuscript.

Conflicts of Interest: The authors declare no conflict of interest.

\section{References}

1. Urbina, J.A. Specific Chemotherapy of Chagas Disease: Relevance, Current Limitations and New Approaches. Acta Trop. 2010, 115, 55-68. [CrossRef] [PubMed]

2. Urbina, J.A. New Insights in Chagas Disease Treatment. Drugs Future 2010, 35, 409-420. [CrossRef]

3. Docampo, R.; Moreno, S. Bisphosphonates as Chemotherapeutic Agents Against Trypanosomatid and Apicomplexan Parasites. Curr. Drug Targets Infect. Disord. 2001, 1, 51-61. [CrossRef] [PubMed]

4. Eberl, M.; Hintz, M.; Reichenberg, A.; Kollas, A.-K.; Wiesner, J.; Jomaa, H. Microbial Isoprenoid Biosynthesis and Human $\gamma \delta$ T Cell Activation. FEBS Lett. 2003, 544, 4-10. [CrossRef]

5. Moreno, S.N.J.; Li, Z. Targeting the Isoprenoid Pathway of Toxoplasma gondii. Expert Opin. Ther. Targets 2008, 12, 253-264. [CrossRef] [PubMed]

6. Nair, S.C.; Brooks, C.F.; Goodman, C.D.; Strurm, A.; McFadden, G.I.; Sundriyal, S.; Anglin, J.L.; Song, Y.; Moreno, S.N.J.; Striepen, B. Apicoplast Isoprenoid Precursor Synthesis and the Molecular Basis of Fosmidomycin Resistance in Toxoplasma gondii. J. Exp. Med. 2011, 208, 1547-1559. [CrossRef] [PubMed]

7. Ling, Y.; Li, Z.H.; Miranda, K.; Oldfield, E.; Moreno, S.N.J. The Farnesyl-Diphosphate/ geranylgeranyl-Diphosphate Synthase of Toxoplasma gondii Is a Bifunctional Enzyme and a Molecular Target of Bisphosphonates. J. Biol. Chem. 2007, 282, 30804-30816. [CrossRef] [PubMed]

8. Li, Z.H.; Cintrón, R.; Koon, N.A.; Moreno, S.N.J. The N-Terminus and the Chain-Length Determination Domain Play a Role in the Length of the Isoprenoid Product of the Bifunctional Toxoplasma gondii Farnesyl Diphosphate Synthase. Biochemistry 2012, 51, 7533-7540. [CrossRef] [PubMed]

9. Li, Z.H.; Ramakrishnan, S.; Striepen, B.; Moreno, S.N.J. Toxoplasma gondii Relies on Both Host and Parasite Isoprenoids and Can Be Rendered Sensitive to Atorvastatin. PLoS Pathog. 2013, 9, e1003665. [CrossRef] [PubMed]

10. Rodriguez, J.B.; Szajnman, S.H. New Antibacterials for the Treatment of Toxoplasmosis; a Patent Review. Expert Opin. Ther. Pat. 2012, 22, 311-333. [CrossRef] [PubMed]

11. Rodan, G.A. Machanisms of Action of Bisphosphonates. Annu. Rev. Pharmacol. Toxicol. 1998, 38, 375-388. [CrossRef] [PubMed]

12. Russell, R.G.G. Bisphosphonates: The First 40 Years. Bone 2011, 49, 2-19. [CrossRef] [PubMed]

13. Reddy, R.; Dietrich, E.; Lafontaine, Y.; Houghton, T.J.; Belanger, O.; Dubois, A.; Arhin, F.F.; Sarmiento, I.; Fadhil, I.; Laquerre, K.; et al. Bisphosphonated Benzoxazinorifamycin Prodrugs for the Prevention and Treatment of Osteomyelitis. ChemMedChem 2008, 3, 1863-1868. [CrossRef] [PubMed]

14. Miller, K.; Erez, R.; Segal, E.; Shabat, D.; Satchi-Fainaro, R. Targeting Bone Metastases with a Bispecific Anticancer and Antiangiogenic Polymer-Alendronate-Taxane Conjugate. Angew. Chem. Int. Ed. 2009, 48, 2949-2954. [CrossRef] [PubMed]

15. Zhang, Y.; Cao, R.; Yin, F.; Hudock, M.P.; Guo, R.T.; Krysiak, K.; Mukherjee, S.; Gao, Y.G.; Robinson, H.; Song, Y.; et al. Lipophilic Bisphosphonates as Dual Farnesyl/geranylgeranyl Diphosphate Synthase Inhibitors: An X-ray and Nmr Investigation. J. Am. Chem. Soc. 2009, 131, 5153-5162. [CrossRef] [PubMed]

16. Roth, A.G.; Drescher, D.; Yang, Y.; Redmer, S.; Uhlig, S.; Arenz, C. Potent and Selective Inhibition of Acid Sphingomyelinase by Bisphosphonates. Angew. Chem. Int. Ed. 2009, 48, 7560-7563. [CrossRef] [PubMed] 
17. Sanders, J.M.; Ghosh, S.; Chan, J.M.W.; Meints, G.; Wang, H.; Raker, A.M.; Song, Y.; Colantino, A.; Burzynska, A.; Kafarski, P.; et al. Quantitative Structure-Activity Relationships for $\gamma \delta$ T Cell Activation by Bisphosphonates. J. Med. Chem. 2004, 47, 375-384. [CrossRef] [PubMed]

18. Liñares, G.E.G.; Ravaschino, E.L.; Rodriguez, J.B. Progresses in the Field of Drug Design to Combat Tropical Protozoan Parasitic Diseases. Curr. Med. Chem. 2006, 13, 335-360. [CrossRef] [PubMed]

19. Urbina, J.A.; Moreno, B.; Vierkotter, S.; Oldfield, E.; Payares, G.; Sanoja, C.; Bailey, B.N.; Yan, W.; Scott, D.A.; Moreno, S.N.J.; et al. Trypanosoma cruzi Contains Major Pyrophosphate Stores, and Its Growth in Vitro and in Vivo Is Blocked by Pyrophosphate Analogs. J. Biol. Chem. 1999, 274, 33609-33615. [CrossRef] [PubMed]

20. Martin, M.B.; Grimley, J.S.; Lewis, J.C.; Heath, H.T.; Bailey, B.N.; Kendrick, H.; Yardley, V.; Caldera, A.; Lira, R.; Urbina, J.A.; et al. Bisphosphonates Inhibit the Growth of Trypanosoma brucei, Trypanosoma cruzi, Leishmania donovani, Toxoplasma gondii, and Plasmodium falciparum: A Potential Route to Chemotherapy. J. Med. Chem. 2001, 44, 909-916. [CrossRef] [PubMed]

21. Martin, M.B.; Sanders, J.M.; Kendrick, H.; de Luca-Fradley, K.; Lewis, J.C.; Grimley, J.S.; Van Brussel, E.M.; Olsen, J.R.; Meints, G.A.; Burzynska, A.; et al. Activity of Bisphosphonates against Trypanosoma brucei rhodesiense. J. Med. Chem. 2002, 45, 2904-2914. [CrossRef] [PubMed]

22. Yardley, V.; Khan, A.A.; Martin, M.B.; Slifer, T.R.; Araujo, F.G.; Moreno, S.N.J.; Docampo, R.; Croft, S.L.; Oldfield, E. In Vivo Activities of Farnesyl Pyrophosphate Synthase Inhibitors against Leishmania donovani and Toxoplasma gondii. Antimicrob. Agents Chemother. 2002, 46, 929-931. [CrossRef] [PubMed]

23. Garzoni, L.R.; Waghabi, M.C.; Baptista, M.M.; de Castro, S.L.; Meirelles, M.D.N.L.; Britto, C.C.; Docampo, R.; Oldfield, E.; Urbina, J.A. Antiparasitic Activity of Risedronate in a Murine Model of Acute Chagas' Disease. Int. J. Antimicrob. Agents 2004, 23, 286-290. [CrossRef] [PubMed]

24. Bouzahzah, B.; Jelicks, L.A.; Morris, S.A.; Weiss, L.M.; Tanowitz, H.B. Risedronate in the Treatment of Murine Chagas' Disease. Parasitol. Res. 2005, 96, 184-187. [CrossRef] [PubMed]

25. Luckman, S.P.; Hughes, D.E.; Coxon, F.P.; Graham, R.; Russell, G.; Rogers, M.J. Nitrogen-Containing Bisphosphonates Inhibit the Mevalonate Pathway and Prevent Post-Translational Prenylation of GTP-Binding Proteins, Including Ras. J. Bone Miner. Res. 1998, 13, 581-589. [CrossRef] [PubMed]

26. Sun, S.T.; McKenna, C.E. Farnesyl Pyrophosphate Synthase Modulators: A Patent Review (2006-2010). Expert Opin. Ther. Pat. 2011, 21, 1433-1451. [CrossRef] [PubMed]

27. Docampo, R.; Moreno, S.N.J. Acidocalcisomes. Cell Calcium 2011, 50, 113-119. [CrossRef] [PubMed]

28. Docampo, R.; de Souza, W.; Miranda, K.; Rohloff, P.; Moreno, S.N.J. Acidocalcisomes-Conserved from Bacteria to Man. Nat. Rev. Microbiol. 2005, 3, 251-261. [CrossRef] [PubMed]

29. Szajnman, S.H.; Bailey, B.N.; Docampo, R.; Rodriguez, J.B. Bisphosphonates Derived from Fatty Acids Are Potent Growth Inhibitors of Trypanosoma cruzi. Bioorg. Med. Chem. Lett. 2001, 11, 789-792. [CrossRef]

30. Szajnman, S.H.; Montalvetti, A.; Wang, Y.; Docampo, R.; Rodriguez, J.B. Bisphosphonates Derived from Fatty Acids Are Potent Inhibitors of Trypanosoma cruzi Farnesyl Pyrophosphate Synthase. Bioorg. Med. Chem. Lett. 2003, 13, 3231-3235. [CrossRef]

31. Szajnman, S.H.; Ravaschino, E.L.; Docampo, R.; Rodriguez, J.B. Synthesis and Biological Evaluation of 1-Amino-1,1-bisphosphonates Derived from Fatty Acids against Trypanosoma cruzi Targeting Farnesyl Pyrophosphate Synthase. Bioorg. Med. Chem. Lett. 2005, 15, 4685-4690. [CrossRef] [PubMed]

32. Ling, Y.; Sahota, G.; Odeh, S.; Chan, J.M.W.; Araujo, F.G.; Moreno, S.N.J.; Oldfield, E. Bisphosphonate Inhibitors of Toxoplasma gondii Growth: In Vitro, QSAR, and in Vivo Investigations. J. Med. Chem. 2005, 48, 3130-3140. [CrossRef] [PubMed]

33. Szajnman, S.H.; Rosso, V.S.; Malayil, L.; Smith, A.; Moreno, S.N.J.; Docampo, R.; Rodriguez, J.B. 1-(Fluoroalkylidene)-1,1-bisphosphonic Acids Are Potent and Selective Inhibitors of the Enzymatic Activity of Toxoplasma gondii Farnesyl Pyrophosphate Synthase. Org. Biomol. Chem. 2012, 10, 1424-1433. [CrossRef] [PubMed]

34. Szajnman, S.H.; García Liñares, G.E.; Li, Z.H.; Jiang, C.; Galizzi, M.; Bontempi, E.J.; Ferella, M.; Moreno, S.N.J.; Docampo, R.; Rodriguez, J.B. Synthesis and Biological Evaluation of 2-Alkylaminoethyl-1,1-bisphosphonic Acids against Trypanosoma cruzi and Toxoplasma gondii Targeting Farnesyl Diphosphate Synthase. Bioorg. Med. Chem. 2008, 16, 3283-3290. [CrossRef] [PubMed] 
35. Rosso, V.S.; Szajnman, S.H.; Malayil, L.; Galizzi, M.; Moreno, S.N.J.; Docampo, R.; Rodriguez, J.B. Synthesis and Biological Evaluation of New 2-Alkylaminoethyl-1,1-bisphosphonic Acids against Trypanosoma cruzi and Toxoplasma gondii Targeting Farnesyl Diphosphate Synthase. Bioorg. Med. Chem. 2011, 19, 2211-2217. [CrossRef] [PubMed]

36. Cinque, G.M.; Szajnman, S.H.; Zhong, L.; Docampo, R.; Schvartzapel, A.J.; Rodriguez, J.B.; Gros, E.G. Structure-Activity Relationship of New Growth Inhibitors of Trypanosoma cruzi. J. Med. Chem. 1998, 41, 1540-1554. [CrossRef] [PubMed]

37. Ferrer-Casal, M.; Li, C.; Galizzi, M.; Stortz, C.A.; Szajnman, S.H.; Docampo, R.; Moreno, S.N.J.; Rodriguez, J.B. New Insights into Molecular Recognition of 1,1-Bisphosphonic Acids by Farnesyl Diphosphate Synthase. Bioorg. Med. Chem. 2014, 22, 398-405. [CrossRef] [PubMed]

38. Recher, M.; Barboza, A.P.; Li, Z.-H.; Galizzi, M.; Ferrer-Casal, M.; Szajnman, S.H.; Docampo, R.; Moreno, S.N.J.; Rodriguez, J.B. Design, Synthesis and Biological Evaluation of Sulfur-Containing 1,1-Bisphosphonic Acids as Antiparasitic Agents. Eur. J. Med. Chem. 2013, 60, 431-440. [CrossRef] [PubMed]

39. Aripirala, S.; Szajnman, S.H.; Jakoncic, J.; Rodriguez, J.B.; Docampo, R.; Gabelli, S.B.; Amzel, L.M. Design, Synthesis, Calorimetry, and Crystallographic Analysis of 2-Alkylaminoethyl-1,1-bisphosphonates as Inhibitors of Trypanosoma cruzi Farnesyl Diphosphate Synthase. J. Med. Chem. 2012, 55, 6445-6454. [CrossRef] [PubMed]

40. Hosfield, D.J.; Zhang, Y.; Dougan, D.R.; Broun, A.; Tari, L.W.; Swanson, R.V.; Finn, J. Structural Basis for Bisphosphonate-Mediated Inhibition of Isoprenoid Biosynthesis. J. Biol. Chem. 2004, 279, 8526-8529. [CrossRef] [PubMed]

41. Huang, C.-H.; Gabelli, S.B.; Oldfield, E.; Amzel, L.M. Binding of Nitrogen-Containing Bisphosphonates (N-BPs) to the Trypanosoma cruzi Farnesyl Diphosphate Synthase Homodimer. Proteins 2010, 78, 888-899. [CrossRef] [PubMed]

42. Cao, R.; Chen, C.K.M.; Guo, R.T.; Wang, A.H.J.; Oldfield, E. Structures of a Potent Phenylalkyl Bisphosphonate Inhibitor Bound to Farnesyl and Geranylgeranyl Diphosphate Synthases. Proteins Struct. Funct. Genet. 2008, 73, 431-439. [CrossRef] [PubMed]

43. Rodriguez, J.B. Tetraethyl Vinylidenebisphosphonate: A Versatile Synthon for the Preparation of Bisphosphonates. Synthesis 2014, 46, 1129-1142. [CrossRef]

44. Mckenna, C.E.; Higa, M.T.; Cheung, N.H.; Mckenna, M. The Facile Dealkylation of Phosphonic Acid Dialkyl Esters by Bromotrimethylsilane. Tetrahedron Lett. 1977, 18, 155-158. [CrossRef]

45. McKenna, C.E.; Schmidhuser, J. Functional Selectivity in Phosphonate Ester Dealkylation with Bromotrimethylsilane. J. Chem. Soc. Chem. Commun. 1979. [CrossRef]

46. Mori, K.; Ueda, H. Synthesis of the Optically Active Forms of 2,6-Dimethyl-1,5-heptadien-3-ol Acetate, the Pheromone of the Comstock Mealybug. Tetrahedron 1981, 37, 2581-2583. [CrossRef]

47. Wicek, M.; Kottke, T.; Ligneau, X.; Schunack, W.; Seifert, R.; Stark, H.; Handzlik, J.; Kieć-Kononowicz, K. N-Alkenyl and Cycloalkyl Carbamates as Dual Acting Histamine H3 and H4 Receptor Ligands. Bioorg. Med. Chem. 2011, 19, 2850-2858. [CrossRef] [PubMed]

48. Wiemer, A.J.; Yu, J.S.; Lamb, K.M.; Hohl, R.J.; Wiemer, D.F. Mono- and Dialkyl Isoprenoid Bisphosphonates as Geranylgeranyl Diphosphate Synthase Inhibitors. Bioorg. Med. Chem. 2008, 16, 390-399. [CrossRef] [PubMed]

49. Duncan, G.D.; Li, Z.; Khare, A.B.; Mckenna, C.E. Oxiranylidene-2,2-bis(phosphonate): Unambiguous Synthesis, Hydrolysis to 1,2-Dihydroxyethylidene-1,1-bis(phosphonate), and Identification as the Primary Product from Mild $\mathrm{Na}_{2} \mathrm{WO}_{4} / \mathrm{H}_{2} \mathrm{O}_{2}$ Oxidation of Ethenylidene-1,1-bis(phosphonate). J. Org. Chem. 1995, 60, 7080-7081. [CrossRef]

50. Shevchuk, M.V.; Sotiropoulos, J.M.; Miqueu, K.; Romanenko, V.D.; Kukhar, V.P. Tetrakis(trimethylsilyl) Ethenylidene-1,1-bisphosphonate: A Mild and Convenient Michael Acceptor for the Synthesis of 2-Aminoethylidene-1,1-bisphosphonic Acids and Their Potassium Salts. Synlett 2011, 2, 1370-1374.

51. Uehara, K.; Tomooka, K. Planar Chiral Organosulfur Cycles. Chem. Lett. 2009, 38, 1028-1029. [CrossRef]

52. Lal, G.S.; Pez, G.P.; Syvret, R.G. Electrophilic NF Fluorinating Agents. Chem. Rev. 1996, 96, 1737-1756. [CrossRef] [PubMed]

53. Xu, Y.; Qian, L.; Prestwich, G.D. Synthesis of Alpha-Fluorinated Phosphonates from Alpha-Fluorovinylphosphonates: A New Route to Analogues of Lysophosphatidic Acid. Org. Lett. 2003, 5, 2267-2270. [CrossRef] [PubMed] 
54. Beier, P.; Opekar, S.; Zibinsky, M.; Bychinskaya, I.; Prakash, G.K.S. A New Route to $\alpha$-Alkyl- $\alpha$ Fluoromethylenebisphosphonates. Org. Biomol. Chem. 2011, 9, 4035-4038. [CrossRef] [PubMed]

55. Marma, M.S.; Xia, Z.; Stewart, C.; Coxon, F.; Dunford, J.E.; Baron, R.; Kashemirov, B.A.; Ebetino, F.H.; Triffitt, J.T.; Russell, R.G.G.; et al. Synthesis and Biological Evaluation of Alpha-Halogenated Bisphosphonate and Phosphonocarboxylate Analogues of Risedronate. J. Med. Chem. 2007, 50, 5967-5975. [CrossRef] [PubMed]

Sample Availability: Samples of the compounds are available from the authors.

(C) 2017 by the authors; licensee MDPI, Basel, Switzerland. This article is an open access article distributed under the terms and conditions of the Creative Commons Attribution (CC-BY) license (http://creativecommons.org/licenses/by/4.0/). 